\title{
The endothelial glycocalyx in early atherogenesis : role in platelet adhesion?
}

Citation for published version (APA):

Reitsma, S. (2012). The endothelial glycocalyx in early atherogenesis : role in platelet adhesion? [Doctoral Thesis, Maastricht University]. Datawyse / Universitaire Pers Maastricht.

https://doi.org/10.26481/dis.20120607sr

Document status and date:

Published: 01/01/2012

DOI:

10.26481/dis.20120607sr

Document Version:

Publisher's PDF, also known as Version of record

\section{Please check the document version of this publication:}

- A submitted manuscript is the version of the article upon submission and before peer-review. There can be important differences between the submitted version and the official published version of record.

People interested in the research are advised to contact the author for the final version of the publication, or visit the DOI to the publisher's website.

- The final author version and the galley proof are versions of the publication after peer review.

- The final published version features the final layout of the paper including the volume, issue and page numbers.

Link to publication

\footnotetext{
General rights rights.

- You may freely distribute the URL identifying the publication in the public portal. please follow below link for the End User Agreement:

www.umlib.nl/taverne-license

Take down policy

If you believe that this document breaches copyright please contact us at:

repository@maastrichtuniversity.nl

providing details and we will investigate your claim.
}

Copyright and moral rights for the publications made accessible in the public portal are retained by the authors and/or other copyright owners and it is a condition of accessing publications that users recognise and abide by the legal requirements associated with these

- Users may download and print one copy of any publication from the public portal for the purpose of private study or research.

- You may not further distribute the material or use it for any profit-making activity or commercial gain

If the publication is distributed under the terms of Article $25 \mathrm{fa}$ of the Dutch Copyright Act, indicated by the "Taverne" license above, 
The endothelial glycocalyx in early atherogenesis

$$
\text { Role in platelet adhesion? }
$$


ISBN 9789461591517

(C) copyright Sietze Reitsma, Maastricht 2012

Printing by Datawyse / Universitaire Pers Maastricht

Cover photo "Missing link" by Hanneke Reitsma at the Reuver-Kessel ferry 


\title{
The endothelial glycocalyx
}

\section{in early atherogenesis}

\author{
Role in platelet adhesion?
}

\author{
PROEFSCHRIFT \\ ter verkrijging van de graad van doctor aan de Universiteit Maastricht, \\ op gezag van de Rector Magnificus, Prof. mr. G.P.M.F. Mols \\ volgens het besluit van het College van Decanen, \\ in het openbaar te verdedigen \\ op donderdag 7 juni 2012 om 12.00 uur \\ door Sietze Reitsma \\ geboren op 24 oktober 1982 te Amsterdam
}

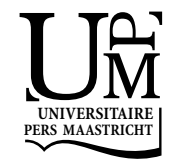




\section{Promotores:}

Prof. dr. D.W. Slaaf

Prof. dr. M.A.M.J. van Zandvoort

Prof. dr. M.G.A. oude Egbrink

\section{Beoordelingscommissie:}

Prof. dr. H. ten Cate (voorzitter)

Prof. dr. E.A.L. Biessen

Dr. M.R. Losen

Prof. dr. A.J. Rabelink (Universiteit Leiden)

Prof. dr. G.W.J.M. Tangelder (Vrije Universiteit Amsterdam)

Financial support by the Dutch Heart Foundation for the publication of this thesis is gratefully acknowledged.

Additional funding by ALK-Abelló, Atos Medical, Daleco Pharma, GlaxoSmithKline, Stallergenes, and Van Boxtel hoorwinkels is gratefully recognized.

The scientific work presented in this thesis was supported by SenterNovem of the Dutch Ministry of Economic Affairs (BSIK 03033). 
“Alles wat glycocalyx heeft, love de Here!"

Naar Psalm 150:6 



\section{Contents}

1 General introduction 9

2 The endothelial glycocalyx: composition, functions and visualization 17

3 Endothelial glycocalyx structure in the intact carotid artery: 49 a two-photon laser scanning microscopy study

$4 \quad$ Endothelial glycocalyx thickness and platelet-vessel wall interactions during atherogenesis

5 Platelet-vessel wall interactions in the isolated mouse carotid artery: roles for flow profile entrance length and endothelial glycocalyx

6 In vivo high-resolution structural imaging of large arteries in small rodents using two-photon laser scanning microscopy

7 General discussion

8 Summary

Samenvatting

About the author / List of publications

Dankwoord 

General introduction 
10 Chapter 1 
Circulation of blood is of vital importance to deliver oxygen and nutrients to tissues, to remove waste products of cell and organ metabolism, and to transport blood cells. The blood cells contribute to various processes, such as oxygen transport (red blood cells) and immune system responses (white blood cells). Platelets are the smallest blood cells in the circulation. They are anuclear and have a life span of 5 - 9 days. At sites of vascular damage platelets become activated, form aggregates, and aid the process of coagulation. ${ }^{1}$ Platelets also have the capacity to adhere to the endothelium, even when there is no evident damage to the vessel wall. $^{2,3}$ As such, platelets have been shown to be involved in other pathophysiological conditions as well. One of these conditions is atherosclerosis.

Atherosclerosis is an inflammatory disease of larger arteries that is marked by the formation of so-called plaques in the vessel wall (atherogenesis). ${ }^{4,5}$ These plaques typically develop under conditions of hyperlipidemia, and localize to sites exposed to low shear stress and reflow, such as arterial bifurcations. ${ }^{6}$ During early atherogenesis, increasing numbers of platelets adhere to the vessel wall of the carotid artery, while prevention of these adhesions dramatically reduces the atherosclerotic burden. 7,8 Observations like these raise the question what property of the endothelium governs the likelihood of platelet adhesion and 'shields' the endothelium from contact with platelets under normal conditions. In this thesis, we explore the hypothesis that the endothelial glycocalyx acts as a modulator of platelet adhesion, and that changes in the endothelial glycocalyx contribute to early atherogenesis.

The endothelial glycocalyx is the carbohydrate-rich lining of endothelial cells. It forms the interface between flowing blood and the endothelium. ${ }^{9}$ Some decades ago, it was first visualized using electron microscopy, and depicted as a very thin (several nanometers) layer produced by the endothelial cells on their luminal surface. ${ }^{10}$ With improving abilities to preserve this delicate structure, its reported thickness increased to some hundreds of nanometers and higher, both in vivo and ex vivo in various vascular beds. ${ }^{11-13}$ An overview of this progress is given in a recent paper by the group of Tarbell, who imaged a glycocalyx of 11 micrometer on cultured endothelial cells, ${ }^{14}$ although the physiological relevance of this finding can be disputed. ${ }^{15}$ Given its location and its (in vivo) dimensions, it is more than likely that the endothelial glycocalyx is involved in many vascular processes, amongst which the control of blood cell-vessel wall interactions. 
A common hypothesis regarding the role of the endothelial glycocalyx in atherogenesis is that it becomes thinner due to atherogenic stimuli, such as hyperlipidemia. ${ }^{16}$ Reduction of endothelial glycocalyx thickness results in an increased likelihood of platelet and leukocyte adhesion, and in increased permeability. ${ }^{17-19}$ These consequences in turn contribute to the initiation of an inflammatory response and, hence, the formation of a plaque. This process only takes place at predilection sites for atherosclerosis. ${ }^{20}$

There are several reports in literature in favor of this hypothesis. (1) The thickness of the endothelial glycocalyx in the carotid artery sinus (a predilection site for atherosclerosis) is reduced. As a consequence, it is less able to maintain a permeability barrier than lesion-protected regions, even in nonatherogenic animals. ${ }^{16,20}$ (2) In hamster cremaster muscle capillaries, the infusion of oxidized low density lipoprotein (ox-LDL; an atherogenic stimulus) degrades the endothelial glycocalyx, leading to an increase in platelet adhesion to the vessel wall. ${ }^{17} \mathrm{~A}$ comparable approach in mouse cremaster venules shows that diet-induced hyperlipidemia decreases endothelial glycocalyx thickness and increases transvascular leakage of lipoproteins. ${ }^{18}$ In the same model, increased leukocyte adhesion to the vessel wall can be observed after degradation of the endothelial glycocalyx by ox-LDL infusion, while restoration of the glycocalyx impairs these interactions. ${ }^{19}$ (3) Chronic inhibition of the synthesis of hyaluronan, a key element of the endothelial glycocalyx, leads to accelerated atherosclerosis and a prothrombotic state of the endothelium. $^{21}$

However, these results are based on different animal models of atherosclerosis and obtained in various tissues. It can be debated whether data from the microcirculation can be extrapolated to the macrocirculation. Up to now, there is no study describing the direct relation between endothelial glycocalyx thickness and likelihood of platelet adhesion during early atherogenesis.

To investigate the role of the endothelial glycocalyx in atherogenesis, an imaging technique is needed that enables reliable visualization and quantification of this layer in intact larger arteries in the course of atherogenesis. Currently used techniques are only partially suited to this end. Electron microscopy requires extensive vessel processing and slicing, resulting in dehydration of the endothelial glycocalyx. ${ }^{10,12}$ Intravital microscopy to image the glycocalyx can only be performed in small vessels, ${ }^{13,17-19}$ as image resolution in larger arteries suffers greatly from scattering of light when imaging through the vessel wall. Confocal microscopy also suffers from deterioration of resolution deeper in tissue. ${ }^{22} \mathrm{~A}$ possible solution to this problem is to visualize the glycocalyx from the luminal side by excising and opening 
the vessel, which damages the integrity of the artery and results in an unpressurized state during imaging. ${ }^{20}$

In contrast, two-photon laser scanning microscopy (TPLSM) can be used to image vascular structures at subcellular resolution in excised, intact, mounted carotid arteries of mice in a pressurized state. ${ }^{23}$ TPLSM is an advanced microscopy technique, depending on the excitation of a fluorophore by simultaneous arrival of two photons of roughly half the energy of that in conventional fluorescence microscopy. As the chance of two-photon excitation is very small outside the focal point of the microscope, both excitation and emission occur in the focal point only. The use of photons with half the energy (i.e., a double wavelength of about $800 \mathrm{~nm}$ ) gives rise to less scattering of excitation light, leading to better conservation of the volume of excitation with increasing tissue depths and, hence, better tissue penetration. As excitation occurs in the focal point only, all signal received originates from the same excited volume, regardless of the amount of scattering of emission light. Furthermore, TPLSM hardly leads to photo-bleaching in areas outside the focal point as seen in other fluorescence microscopy techniques. Some reports describing the basics of TPLSM and its application in vascular research are recommended for further reading. ${ }^{23-29}$ Because of the above-mentioned advantages, TPLSM was used to image the delicate endothelial glycocalyx in isolated, intact carotid arteries of mice. The use of TPLSM in larger arteries in vivo, however, is complicated by the fact that there is vessel motion during the point-by-point image acquisition, leading to image distortion. Therefore, in this thesis, in vivo plateletvessel wall interactions in the course of atherogenesis was studied using 'regular' intravital fluorescence microscopy (IFM). ${ }^{30}$

The aim of this thesis was to describe the role of the endothelial glycocalyx in platelet adhesion during early atherogenesis. In Chapter 2, the current knowledge on endothelial glycocalyx structure, function and visualization techniques is reviewed in detail. Chapter 3 describes reliable endothelial glycocalyx visualization and quantification in mouse carotid arteries using TPLSM. In Chapter 4, both platelet-vessel wall interactions (IFM) and endothelial glycocalyx thickness (TPLSM) are explored in the common carotid artery and carotid bifurcation of atherogenic mice and wildtype controls. In Chapter 5, platelet-vessel wall interactions are assessed in the isolated mouse carotid artery, exploring the role of flow profiles and the endothelial glycocalyx in platelet adhesion. Chapter 6 describes the feasibility of in vivo stable imaging of small structures in the vessel wall of larger arteries using TPLSM. The main findings of the work presented in this thesis are discussed in Chapter 7. 


\section{References}

1. oude Egbrink MG, Van Gestel MA, Broeders MA, Tangelder GJ, Heemskerk JM, Reneman RS, Slaaf DW. Regulation of microvascular thromboembolism in vivo. Microcirculation. 2005;12:287-300.

2. Leslie M. Cell biology. Beyond clotting: the powers of platelets. Science. 2010;328:562-564.

3. Wagner DD, Frenette PS. The vessel wall and its interactions. Blood. 2008;111:5271-5281.

4. Libby P. Inflammation in atherosclerosis. Nature. 2002;420:868-874.

5. Hansson GK. Inflammation, atherosclerosis, and coronary artery disease. $N$ Engl J Med. 2005;352:1685-1695.

6. Zarins CK, Giddens DP, Bharadvaj BK, Sottiurai VS, Mabon RF, Glagov S. Carotid bifurcation atherosclerosis. Quantitative correlation of plaque localization with flow velocity profiles and wall shear stress. Circ Res. 1983;53:502-514.

7. Massberg S, Brand K, Gruner S, Page S, Muller E, Muller I, Bergmeier W, Richter T, Lorenz M, Konrad I, Nieswandt B, Gawaz M. A critical role of platelet adhesion in the initiation of atherosclerotic lesion formation. J Exp Med. 2002;196:887-896.

8. Huo Y, Schober A, Forlow SB, Smith DF, Hyman MC, Jung S, Littman DR, Weber C, Ley K. Circulating activated platelets exacerbate atherosclerosis in mice deficient in apolipoprotein E. Nat Med. 2003;9:61-67.

9. Reitsma S, Slaaf DW, Vink H, van Zandvoort MA, oude Egbrink MG. The endothelial glycocalyx: composition, functions, and visualization. Pflugers Arch. 2007;454:345-359.

10. Luft JH. Fine structures of capillary and endocapillary layer as revealed by ruthenium red. Fed Proc. 1966;25:1773-1783.

11. Rostgaard J, Qvortrup K. Electron microscopic demonstrations of filamentous molecular sieve plugs in capillary fenestrae. Microvasc Res. 1997;53:1-13.

12. van den Berg BM, Vink H, Spaan JA. The endothelial glycocalyx protects against myocardial edema. Circ Res. 2003;92:592-594.

13. Vink H, Duling BR. Identification of distinct luminal domains for macromolecules, erythrocytes, and leukocytes within mammalian capillaries. Circ Res. 1996;79:581-589.

14. Ebong EE, Macaluso FP, Spray DC, Tarbell JM. Imaging the endothelial glycocalyx in vitro by rapid freezing/freeze substitution transmission electron microscopy. Arterioscler Thromb Vasc Biol. 2011;31:1908-1915.

15. Potter DR, Damiano ER. The hydrodynamically relevant endothelial cell glycocalyx observed in vivo is absent in vitro. Circ Res. 2008;102:770-776.

16. van den Berg BM, Spaan JA, Rolf TM, Vink $H$. Atherogenic region and diet diminish glycocalyx dimension and increase intima-to-media ratios at murine carotid artery bifurcation. Am J Physiol Heart Circ Physiol. 2006;290:H915-920.

17. Vink $\mathrm{H}$, Constantinescu AA, Spaan JA. Oxidized lipoproteins degrade the endothelial surface layer: implications for platelet-endothelial cell adhesion. Circulation. 2000;101:1500-1502.

18. Constantinescu A, Spaan JA, Arkenbout EK, Vink H, Vanteeffelen JW. Degradation of the endothelial glycocalyx is associated with chylomicron leakage in mouse cremaster muscle microcirculation. Thromb Haemost. 2011;105:790-801.

19. Constantinescu AA, Vink H, Spaan JA. Endothelial cell glycocalyx modulates immobilization of leukocytes at the endothelial surface. Arterioscler Thromb Vasc Biol. 2003;23:1541-1547.

20. van den Berg BM, Spaan JA, Vink H. Impaired glycocalyx barrier properties contribute to enhanced intimal low-density lipoprotein accumulation at the carotid artery bifurcation in mice. Pflugers Arch. 2009;457:1199-1206. 
21. Nagy N, Freudenberger T, Melchior-Becker A, Rock K, Ter Braak M, Jastrow H, Kinzig M, Lucke S, Suvorava T, Kojda G, Weber AA, Sorgel F, Levkau B, Ergun S, Fischer JW. Inhibition of hyaluronan synthesis accelerates murine atherosclerosis: novel insights into the role of hyaluronan synthesis. Circulation. 2010;122:2313-2322.

22. van Zandvoort M, Engels W, Douma K, Beckers L, Oude Egbrink M, Daemen M, Slaaf DW. Twophoton microscopy for imaging of the (atherosclerotic) vascular wall: a proof of concept study. $J$ Vasc Res. 2004;41:54-63.

23. Megens RT, Reitsma S, Schiffers PH, Hilgers RH, De Mey JG, Slaaf DW, oude Egbrink MG, van Zandvoort MA. Two-photon microscopy of vital murine elastic and muscular arteries. Combined structural and functional imaging with subcellular resolution. J Vasc Res. 2007;44:87-98.

24. Megens RT, oude Egbrink MG, Merkx M, Slaaf DW, van Zandvoort MA. Two-photon microscopy on vital carotid arteries: imaging the relationship between collagen and inflammatory cells in atherosclerotic plaques. J Biomed Opt. 2008;13:044022.

25. Megens RT, Kemmerich K, Pyta J, Weber C, Soehnlein O. Intravital imaging of phagocyte recruitment. Thromb Haemost. 2011;105:802-810.

26. Masters BR, So PT. Antecedents of two-photon excitation laser scanning microscopy. Microsc Res Tech. 2004;63:3-11.

27. Zoumi A, Lu X, Kassab GS, Tromberg BJ. Imaging coronary artery microstructure using secondharmonic and two-photon fluorescence microscopy. Biophys J. 2004;87:2778-2786.

28. Konig K. Multiphoton microscopy in life sciences. J Microsc. 2000;200 (Pt 2):83-104.

29. Dunn KW, Sandoval RM, Kelly KJ, Dagher PC, Tanner GA, Atkinson SJ, Bacallao RL, Molitoris BA. Functional studies of the kidney of living animals using multicolor two-photon microscopy. Am J Physiol Cell Physiol. 2002;283:C905-916.

30. van Gestel MA, Reitsma S, Slaaf DW, Heijnen VV, Feijge MA, Lindhout T, van Zandvoort MA, Elg M, Reneman RS, Heemskerk JW, oude Egbrink MG. Both ADP and thrombin regulate arteriolar thrombus stabilization and embolization, but are not involved in initial hemostasis as induced by micropuncture. Microcirculation. 2007;14:193-205. 



\section{The endothelial glycocalyx}

\section{Composition, functions and visualization}

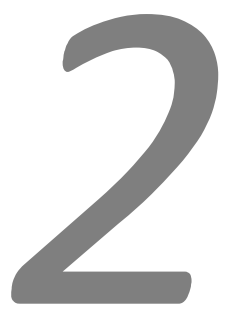

Updated version of

Sietze Reitsma, Dick W. Slaaf, Hans Vink, Marc A.M.J. van Zandvoort, Mirjam G.A. oude Egbrink

Pflügers Archives - European Journal of Physiology, 2007; 454:345-359 


\section{Abstract}

This review aims at presenting state-of-the-art knowledge on the composition and functions of the endothelial glycocalyx. The endothelial glycocalyx is a network of membrane-bound proteoglycans and glycoproteins, covering the endothelium luminally. Both endothelium- and plasma-derived soluble molecules integrate into this mesh. Over the past decade, insight has been gained into the role of the glycocalyx in vascular physiology and pathology, including mechanotransduction, hemostasis, signaling, and blood cell-vessel wall interactions. The contribution of the glycocalyx to diabetes, ischemia/reperfusion and atherosclerosis is also reviewed. Experimental data from the micro- and macrocirculation alludes at a vasculoprotective role for the glycocalyx. Assessing this possible role of the endothelial glycocalyx requires reliable visualization of this delicate layer, which is a great challenge. An overview is given of the various ways in which the endothelial glycocalyx has been visualized up to now, including first data from two-photon microscopic imaging. 


\section{Introduction}

The endothelial glycocalyx was already visualized some 40 years ago by Luft using electron microscopy. ${ }^{1}$ Still, relatively little is known of the composition and function of this layer. Over the past decades, it has been increasingly appreciated as an important factor in vascular physiology and pathology, as described in 2000 in a review by Pries et al., ${ }^{2}$ and in other, more recent reviews. ${ }^{3,4}$ The interest in the (patho)physiological role of the glycocalyx started with the observation of low and variable capillary tube hematocrit, which depended on the level of metabolic and pharmacological activation of the vascular system. ${ }^{5-9}$ The relation between metabolic and agonist-induced increases in red blood cell velocity on the one hand and tube hematocrit on the other could partly be explained by plasma skimming as direct continuation of the Fàhraeus effect. ${ }^{9}$ However, this relation dissociated upon local treatment of the microvessels with heparinase, an enzyme which breaks down heparan sulfates in the glycocalyx. ${ }^{10}$ This finding was in agreement with theoretical estimates predicting a $1.2 \mu \mathrm{m}$ thick slow moving plasma layer over the endothelium. ${ }^{6}$ In vivo studies have revealed the glycocalyx in muscle capillaries to be a layer of about $0.5 \mu \mathrm{m}$ thick, covering endothelial cells and determining luminal domains for macromolecules, red and white blood cells. ${ }^{11}$ More recent studies indicate that glycocalyx thickness increases with vascular diameter, at least in the arterial system, ranging from 2 to $3 \mu \mathrm{m}$ in small arteries ${ }^{12}$ to $4.5 \mu \mathrm{m}$ in carotid arteries. $^{13}$

To date, many studies indicate a variety of (patho)physiological roles for the endothelial glycocalyx; in addition to modulating capillary red blood cell filling, the glycocalyx may affect many other (dys)functions of the vascular system. Whereas the vascular endothelium is currently believed to be actively involved "in every pathology presenting vascular projections", ${ }^{14}$ the same saying might well prove true for the glycocalyx. Assessing this possible involvement of the endothelial glycocalyx requires reliable visualization of this delicate layer, which is a great challenge. This review provides basic insight into the present knowledge of composition and functions of the endothelial glycocalyx and gives an overview of the various ways in which it has been visualized up to now.

\section{Composition}

The endothelial glycocalyx is a carbohydrate-rich layer lining the vascular endothelium. It is considered to be connected to the endothelium through several "backbone" molecules, mainly proteoglycans and also glycoproteins. These form a 


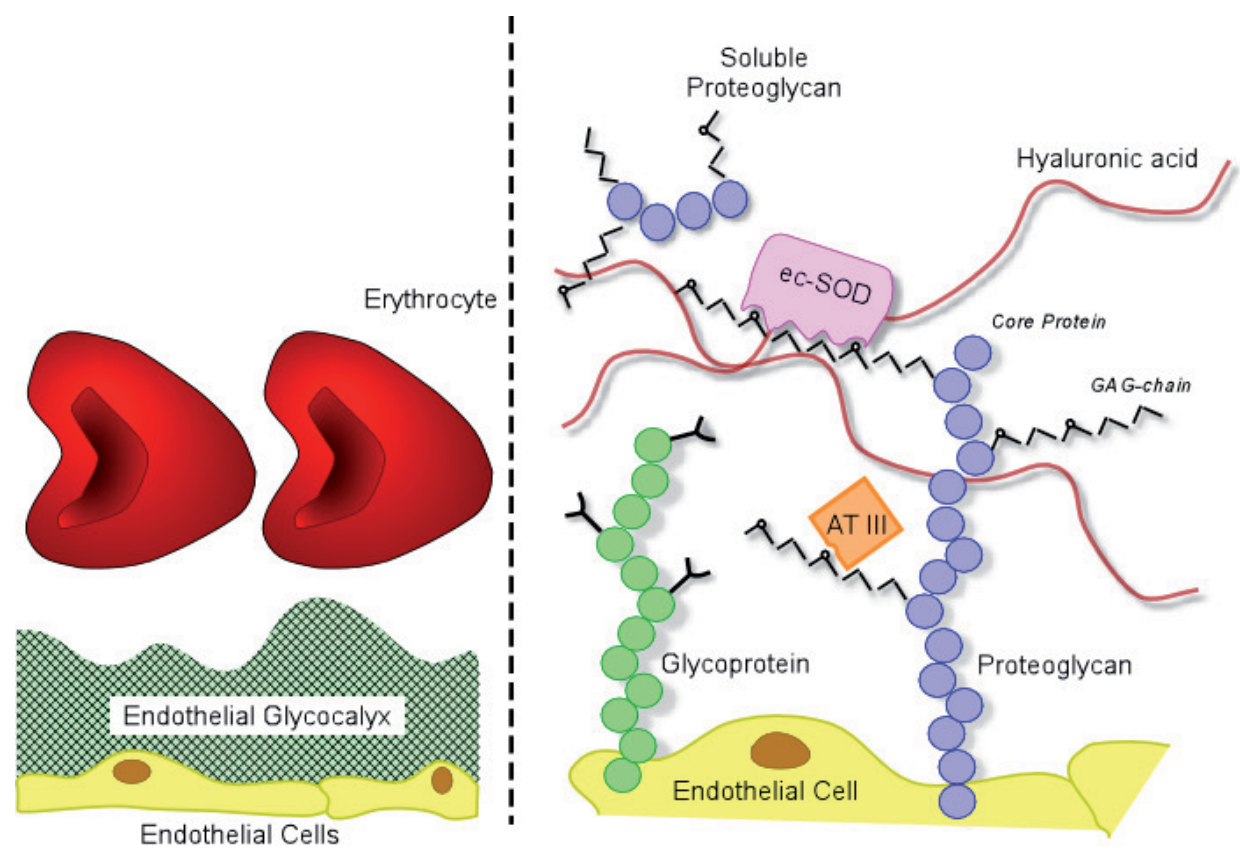

Figure 2.1 Schematic representation of the endothelial glycocalyx, showing its main components.

Left The endothelial glycocalyx can be observed in vivo as a red blood cell exclusion zone, located on the luminal side of the vascular endothelium. It consists of membrane-bound and soluble molecules.

Right Components of the endothelial glycocalyx. Bound to the endothelial membrane are proteoglycans, with long unbranched glycosaminoglycan side chains (GAG-chain) and glycoproteins, with short branched carbohydrate side chains. Incorporated in and on top of this grid are plasma and endotheliumderived soluble components, including hyaluronic acid and other soluble proteoglycans (e.g., thrombomodulin) and various proteins, such as extracellular superoxide dismutase (ec-SOD) and antithrombin III (AT III). Together, these components form the endothelial glycocalyx that functions as a barrier between blood plasma and the endothelium and exerts various roles in plasma and vessel wall homeostasis.

Note that this figure is not drawn to scale; its purpose is to illustrate glycocalyx composition.

network in which soluble molecules, either plasma- or endothelium-derived, are incorporated. More luminally, the glycocalyx is formed by soluble plasma components, linked to each other in a direct way or via soluble proteoglycans and/or glycosaminoglycans (which will be discussed below). A dynamic equilibrium exists between this layer of soluble components and the flowing blood, continuously affecting composition and thickness of the glycocalyx. Furthermore, the glycocalyx suffers from enzymatic or shear-induced shedding. The dynamic balance between biosynthesis and shedding makes it hard to define the glycocalyx geometrically. ${ }^{15}$ The composition of the membrane-bound mesh of proteoglycans, glycoproteins and glycosaminoglycans and the composition of associated plasma proteins and soluble glycosaminoglycans cannot be viewed as a static picture. Instead, the layer 
as a whole - also known as endothelial surface layer (ESL) ${ }^{2}$ - is very dynamic, with membrane-bound molecules being constantly replaced and no distinct boundary between locally synthesized and associated elements; membrane-bound hyaluronan may reach lengths of $>1 \mu \mathrm{m}$. Direct visualization techniques (see section on Visualization techniques below) fail to demonstrate clear compositional differences within the glycocalyx, from endothelial membrane towards vascular lumen, but rather indicate that the endothelial glycocalyx resembles an intricate, selfassembling three-dimensional mesh of various polysaccharides. Enzymatic removal of any of its constituents dramatically affects glycocalyx properties, which exemplifies the importance of considering the synergetic interaction of all glycocalyx constituents as a whole. In this review we will, therefore, use the term (endothelial) glycocalyx for the total layer (Figure 2.1) and be as specific as possible when addressing its various elements.

Below, state-of-the-art knowledge on the various components of the endothelial glycocalyx will be provided. Although many molecules have been identified as being part of the glycocalyx, information on their distribution is still scarce; if present, such knowledge was mostly obtained indirectly and nonquantitatively.

\section{Proteoglycans}

Proteoglycans are generally considered to function as the most important "backbone" molecules of the glycocalyx. They consist of a core protein to which one or more glycosaminoglycan chains are linked. There is a notable variation amongst the proteoglycan core proteins with regard to their size, number of attached glycosaminoglycan chains, and whether or not they are bound to the cell membrane (Table 2.1). The core protein groups of syndecans $(n=4)$ and glypicans $(n=6)$ have a firm connection to the cell membrane via a membrane-spanning domain (syndecans) or a glycosylphosphatidylinositol anchor (glypicans). ${ }^{16,17}$ The other proteoglycans, such as mimecan, perlecan and biglycan, are secreted after their assembly and glycosaminoglycan chain modification. ${ }^{18,19}$ This leads to production of soluble proteoglycans, which reside in the glycocalyx or diffuse into the blood stream.

Proteoglycans are promiscuous in their binding of glycosaminoglycan chains, meaning that one core protein can contain different types of glycosaminoglycan chains. The proportion of the various chains may change under different circumstances and stimuli. ${ }^{20}$ Therefore, naming of proteoglycans after one type of glycosaminoglycan is somewhat deluding. For example, syndecan-1 proteoglycan is often addressed as a heparan sulfate proteoglycan, while in fact, it usually contains similar numbers of heparan sulfate and chondroitin sulfate chains. ${ }^{21}$ 
Table 2.1 Characteristics of proteoglycan core proteins in the vascular endothelial glycocalyx

\begin{tabular}{llllll}
\hline \hline Core protein & & & \multicolumn{3}{l}{ GAG-chains } \\
Group & Relation to membrane & $\begin{array}{l}\text { Size } \\
(\mathrm{kDa})\end{array}$ & $\begin{array}{l}\text { Types } \\
(\mathrm{n})\end{array}$ & $\begin{array}{l}\text { Number } \\
(\mathrm{n})\end{array}$ & Type \\
\hline \hline Syndecan & Membrane-spanning & $19-35$ & 4 & 5 & HS/CS \\
Glypican & GPI-anchor & $57-69$ & 6 & 3 & HS/CS \\
Perlecan & Secreted & 400 & 1 & 3 & HS/CS \\
Versican & Secreted & 370 & 1 & $10-30$ & CS/DS \\
Decorin & Secreted & 40 & 1 & 1 & CS/DS \\
Biglycan & Secreted & 40 & 1 & 2 & CS/DS \\
Mimecan & Secreted & 35 & 1 & $2-3$ & KS \\
\hline $\begin{array}{l}\text { GAG glycosaminoglycan; HS heparan sulfate; CS chondroitin } \\
\text { KS keratan sulfate; GPI glycosylphosphatidylinositol }\end{array}$ & & & dermatan sulfate;
\end{tabular}

There are five types of glycosaminoglycan chains: heparan sulfate, chondroitin sulfate, dermatan sulfate, keratan sulfate, and hyaluronan (or hyaluronic acid). They are linear polymers of disaccharides with variable lengths that are modified by sulfation and/or (de)acetylation to a variable extent. The disaccharides are each composed of a uronic acid and a hexosamine; classification of the glycosaminoglycans depends on which uronic acid or hexosamine is incorporated and on the pattern of sulfation (Table 2.2). Each of the five glycosaminoglycans has been investigated and reviewed extensively ${ }^{22-26}$. Dermatan sulfate is often regarded as a separate class of glycosaminoglycans, although it actually is type B chondroitin sulfate. The difference between the two is possible epimerization of the glucuronic acids into iduronic acids in dermatan sulfate, which has important consequences for functionality. Whenever possible, we will try to separate the two as precisely as possible; elsewhere, they will be referred to as chondroitin sulfate / dermatan sulfate.

In vasculature, heparan sulfate proteoglycans represent roughly $50-90 \%$ of the total amount of proteoglycans present in the glycocalyx. ${ }^{2,27}$ However, this figure is variable, as the expression of proteoglycans by endothelial cells depends on various stimuli. Syndecans, for example, have a tightly regulated expression pattern which varies with endothelial cell activation or stimulation with different chemokines. ${ }^{28}$ The second most common glycosaminoglycan in the endothelial cell glycocalyx is chondroitin sulfate / dermatan sulfate. The presence of heparan sulfate and 
Table 2.2 Composition of the disaccharides of various glycosaminoglycan chains

\begin{tabular}{|c|c|c|c|c|}
\hline & Uronic acid & Disaccharide link & Hexosamine & Polymerization link \\
\hline HS & $\begin{array}{l}\operatorname{GlcA}(2 S) \\
\operatorname{IdoA}(2 S)\end{array}$ & $1 \beta 4$ & $\begin{array}{l}\text { GlcNAc } \\
(N S)(3 S)(6 S)\end{array}$ & $1 \beta 4$ \\
\hline CS* & GlcA & $1 \beta 3$ & $\begin{array}{l}\text { GalNAc4S } \\
\text { GalNAc6S }\end{array}$ & $1 \beta 4$ \\
\hline DS* & $\begin{array}{l}\text { GlcA } \\
\operatorname{IdoA}(2 S)\end{array}$ & $1 \beta 3$ & GalNAc(4S)(6S) & $1 \beta 4$ \\
\hline $\mathrm{HA}$ & GlcA & $1 \beta 3$ & GlcNAc & $1 \beta 4$ \\
\hline KS & Gal(6S) & $1 \beta 4$ & $\operatorname{GlcNAc}(6 \mathrm{~S})$ & $1 \beta 3$ \\
\hline
\end{tabular}

$H A$ hyaluronan; KS keratan sulfate; GICA glucuronic acid; IdoA iduronic acid; Gal galacturonic acid; GlcNAc N-acetyl-glucosamine; GaINAc N-acetyl-galactosamine; 2S 2-O-sulfated; 3S 3-O-sulfated; 4S 4-Osulfated; 6S 6-O-sulfated; NS N-sulfated

Note the various possibilities of sulfation in heparan sulfate. These may coincide (e.g., in heparan sulfate the hexosamine GIcNS3S). A rare but possible hexosamine in heparan sulfate is the N-unsubstituted glucosamine (GlcNH3+), which has been left out of the table for convenient reading. Also note the presence of IdoA in dermatan sulfate, in contrast to the other chondroitin sulfates, making it more alike to heparan sulfate.

*There are three types of chondroitin sulfate. Type A only has 4-O-sulfated $\mathrm{N}$-acetyl-galactosamines, type $\mathrm{B}$ is known as dermatan sulfate and type $\mathrm{C}$ only has 6-O-sulfated $\mathrm{N}$-acetyl-galactosamines.

chondroitin sulfate is reported to have a typical ratio of $4: 1$ for the vascular endothelium. ${ }^{21,29}$ Expression of keratan sulfate glycosaminoglycans in vasculature and its importance in (patho)physiology is less well understood. Another important glycosaminoglycan in the glycocalyx is hyaluronan. This long polymeric molecule (up to $10^{4} \mathrm{kDa}$ ) differs from other glycosaminoglycans in that it is not linked to a core protein. Its exact link to the cell membrane is unknown, but it can be bound to the receptor CD44. ${ }^{30}$ Alternatively, hyaluronan may be attached to its assembly proteins, the hyaluronan synthases, ${ }^{31}$ which are located at the cytosolic side of the cell membrane. Another possibility is that hyaluronan is (in part) not directly bound to the membrane at all. It is capable of forming strikingly viscous solutions. ${ }^{25}$ Furthermore, intracellular hyaluronan binding proteins such as cdc37 ${ }^{32}$ and P32 33 have been identified, suggesting a role for this glycosaminoglycan within the cell. $^{26,34}$

Heparan sulfate and chondroitin sulfate / dermatan sulfate containing proteoglycans are produced in the endoplasmatic reticulum and Golgi apparatus of the endothelial cell. After the ribosomal translation of the core protein, a xylosyltrans- 
ferase will transfer xylose (Xyl) from uracildiphosphate xylose to specific serine residues (Ser) in the core protein. The xylose-enriched core protein is transported to the cis-Golgi and galactosyltransferases type I and II will add two galactosegroups (Gal) to the xylose, after which glucuronosyltransferase type I adds glucuronic acid, thus completing the primary linker for glycosaminoglycan chains: GlcA - $\beta 3-\mathrm{Gal}-\beta 3-\mathrm{Gal}-\beta 4-\mathrm{Xyl} \beta 3-[$ Ser]

After formation of the primary linker, the following step determines the type of glycosaminoglycan chain that will be formed. In case of heparan sulfate, an $\alpha 4$ glucosamine is added; in case of chondroitin sulfate and dermatan sulfate, a $\beta 4$ galactosamine is added and both galactose-residues from the linker may be sulfated. From this point onward, glucuronic acids and glucosamines are linked to the core protein. After chain polymerization, the growing glycosaminoglycan chain will undergo modifications including $\mathrm{N}$-sulfation, O-sulfation and epimerization. The latter changes glucuronic acid residues into iduronic acid residues, and will change any chondroitin sulfate chain into its type B dermatan sulfate. These chain modifications take place in both the cis- and the trans-Golgi, determining the final type and functionality of the proteoglycan and each of its side chains. In contrast, hyaluronan is assembled at the cytosolic side of the cell membrane, and it is not modified afterwards. As a consequence, it has no sulfate groups or modification pattern. As glycosaminoglycan chains contain numerous specific binding sites for plasmaderived proteins, small chain modifications can have great functional consequences. Sequential enzymatic modifications of the individual saccharide units within glycosaminoglycan chains endow proteoglycans with unique functions. Typically 16 - 48 different sulfation patterns may exist per disaccharide and as functional domains are assumed to be usually penta- to deca-saccharide long, at least $16^{3}=4096$ different sulfation patterns on a hexasaccharide backbone are theoretically possible, and such a structural diversity corresponds to diversified biological functions of glycosaminoglycans. Indeed, it has been shown that modification patterns vary in time and under different (patho)physiological stimuli. ${ }^{43}{ }^{35}$ The diversity of glycosaminoglycan sulfation patterns and its effect on specific protein binding and modulation of protein function suggest that conditions that diminish glycocalyx thickness or modulate protein specific glycosaminoglycan sulfation patterns and charge are likely to modulate vascular permeability and alter specific protein binding and activity. 


\section{Glycoproteins}

Beside the proteoglycans with their long linear side chains, certain glycoproteins are also regarded as "backbone" molecules, connecting the glycocalyx to the endothelial cell membrane. This group of endothelial glycoproteins, characterized by relatively small ( 2 - 15 sugar residues) and branched carbohydrate side chains, comprises a number of molecules that all have been studied intensively; major classes that will be discussed in more detail below are the endothelial cell adhesion molecules and components of the coagulation and fibrinolysis system. It is beyond the scope of this review to categorically discuss all glycoproteins that can be expressed by endothelial cells. Furthermore, one should realize that the level of glycoprotein expression on the endothelial cell membrane varies considerably with cell activation or stimulation.

The endothelial cell adhesion molecules are well-defined glycoproteins that play a major role in cell recruitment from the bloodstream and in cell signaling. The three families of cell adhesion molecules present in the endothelial glycocalyx are the selectin family, the integrin family and the immunoglobulin superfamily.

Glycoproteins from the selectin family contain a cytoplasmic tail, a transmembrane domain, several consensus repeats, an epidermal growth factor-like domain, and a terminal lectin domain, which is primarily responsible for binding of carbohydrate groups on glycosylated proteins or lipids. However, the epidermal growth factorlike domain is involved in selectin-ligand recognition as well. ${ }^{36,37}$ Selectins found on the vascular endothelium are E-selectin and P-selectin, both involved in leukocyteendothelial cell interactions. ${ }^{38}$ P-selectin is constitutively produced and subsequently stored in the Weibel-Palade bodies of the endothelial cells. Exocytosis of Weibel-Palade bodies as induced by stimuli such as thrombin and histamine allows a rapid translocation of P-selectin to the cell surface. ${ }^{39,40}$ However, this expression is short-lived due to P-selectin internalization and redirection to lysosomal granules or the Golgi apparatus, where it is restored in newly formed Weibel-Palade bodies. ${ }^{41}$ E-selectin is not stored in granules, but requires de novo mRNA and protein synthesis to be expressed on the cell surface. Stimulation of endothelial cells by cytokines such as interleukin-1, tumor necrosis factor $\alpha$, and lipopolysaccharide upregulates E-selectin expression; this typically requires 2 to $6 \mathrm{~h}^{42}$ In some tissues like the skin, $\mathrm{P}$ - and E-selectin appear to be constitutively expressed on endothelial cells. $^{43,44}$ 
Integrins are heterodimeric molecules, composed of noncovalently bound $\alpha$ and $\beta$ subunits. Both subunits have a cytoplasmic tail and a transmembrane domain, and together, they constitute an integral membrane protein. To date, 18 different $\alpha$ subunits and $8 \beta$-subunits have been identified, which means that each integrin is characterized by the specific combination of its subunits. ${ }^{45}$ Integrins are found on many cell types, including endothelial cells, leukocytes, and platelets. In their luminal membrane, endothelial cells express integrin $\alpha_{v} \beta_{3}$, which is an important mediator of platelet-endothelial cell interactions. ${ }^{46}$ Most other endothelial cell integrins are involved in binding to the basement membrane. These integrins, such as $\alpha_{2} \beta_{1}, \alpha_{5} \beta_{1}$, and $\alpha_{6} \beta_{1}$, bind to multiple extracellular matrix ligands, and are as such, responsible for interactions with laminin, fibronectin, and collagen. Many studies have focused and still focus on the interactions between these integrins and the subendothelial matrix during angiogenesis. ${ }^{47}$

The immunoglobulin superfamily of glycoproteins is characterized by a cytoplasmic tail, a transmembrane domain, and a variable number of immunoglobulin-like domains that protrude luminally. Best known examples are intercellular adhesion molecule 1 and 2 (ICAM-1 and -2), vascular cell adhesion molecule 1 (VCAM-1), and platelet/endothelial cell adhesion molecule 1 (PECAM-1), which act as ligand for integrins on leukocytes and platelets and are crucial mediators of leukocyte homing to the endothelium and subsequent diapedesis. ICAM-1 and 2 and PECAM- 1 have a baseline expression, whereas VCAM-1 is only present after endothelial cell stimulation by cytokines, which also upregulates ICAM-1 expression. ${ }^{48}$ Paradoxically, the constitutive expression of PECAM-1 is decreased after cytokine treatment. ${ }^{49}$ The role of ICAM-2 in inflammation is still unclear, as it is also downregulated by inflammatory stimuli. However, it was shown that ICAM-2 is closely involved in angiogenesis. $^{50}$

Beside the cell adhesion molecules, the endothelial glycocalyx harbors glycoproteins with functionality in coagulation, fibrinolysis, and hemostasis. A good example is the glycoprotein Ib-IX-V complex, which is expressed on endothelial cells and also on platelets. It consists of four glycoproteins: Ib $\alpha, \mathrm{Ib} \beta, \mathrm{IX}$, and $\mathrm{V}$, that are each membrane-spanning polypeptides. Glycoprotein $\mathrm{Ib} \alpha$ and $\mathrm{Ib} \beta$ are covalently linked via a disulfide group, whereas IX and $\mathrm{V}$ are noncovalently attached to the $\mathrm{Ib}$ heterodimer. The Ib-IX-V complex binds von Willebrand factor ( $\mathrm{VWf}$ ) and is primarily known as the platelet vWf-receptor. ${ }^{51,52}$ Furthermore, the complex also binds $\mathrm{P}$ selectin, mediating the interaction of platelets with activated endothelial cells. ${ }^{53}$ Like platelets, endothelial cells express all components of the Ib-IX-V complex, ${ }^{54,55}$ which on one hand, allows binding to the vWf substrate of the subendothelium, 
and on the other, binds Weibel-Palade body derived vWf, secreted luminally by activated endothelial cells.

\section{Soluble components}

Embedded within and layered on top of the mesh of proteoglycans and glycoproteins are soluble components of various types such as proteins and soluble proteoglycans. These components are either derived from the endothelium or from the bloodstream, such as albumin and orosomucoid, which are pivotal in preserving the (charge-)selectivity of the permeability barrier. ${ }^{56}$ The soluble components of the glycocalyx contribute greatly to the functional importance of the glycocalyx, as will be described below. The structural attribution of these soluble components is less well established. At least some of the soluble components may contribute to the structural organization of the luminal glycocalyx, though this is hard to prove. There are indications that proteoglycans bind to each other and to proteins. ${ }^{57,58}$ Protein-glycosaminoglycan-protein complexes have been identified, though not in the glycocalyx in particular. $^{59}$ Still, it is presumable that interactions between membrane-bound proteoglycans, soluble proteins, and soluble proteoglycans create a cross-linked mesh and provide some stability to the luminal glycocalyx. Hyaluronan might play an important role in this respect, being a very large linear molecule and possibly unbound to the endothelial cell membrane (Figure 2.1). It has been shown to interact with itself, forming stable hyaluronan-hyaluronan complexes. $^{60,61}$ Still, the glycocalyx is a delicate layer, and removal of one specific component may result in loss of function of the total. ${ }^{62}$ This is illustrated by experiments by van den Berg et al., showing that specific enzymatic removal of hyaluronan from the glycocalyx induces loss of heparan sulfates as well. ${ }^{63}$

\section{Functional importance}

\section{The endothelial glycocalyx as the endothelial gatekeeper}

Located between the blood stream and the endothelium, the endothelial glycocalyx is an important determinant of vascular permeability. ${ }^{64,65}$ It is able to limit access of certain molecules to the endothelial cell membrane, as has been demonstrated in small rat mesenteric arteries with the use of fluorescently labeled dextrans of various molecular weights, showing increasing permeability for smaller molecules. ${ }^{12}$ Enzymatic (partial) removal and subsequent loss of permeability barrier function of the glycocalyx in rat myocardial capillaries leads to myocardial edema. ${ }^{66}$ Conversely, retention of hydroxyethyl starch molecules within the glycocalyx leads to decreased permeability for small dextrans. ${ }^{67}$ Not only size and steric 
hindrance play a role in glycocalyx dependent permeability, but also, electrostatic charges of the glycocalyx and the permeating substance. With many of the glycosaminoglycan chains being highly sulfated, the glycocalyx presents a net negatively charged surface to the bloodstream. Accordingly, neutralization of the glycocalyx induces an increase in albumin uptake by cultured endothelial cells ${ }^{68}$ and an increased permeability for fluorescently labeled dextrans in rat mesenteric arteries. ${ }^{69}$

Vascular permeability is an important factor in the physiology of, for example, the kidneys. Several reports describe how damage to the glycocalyx induces proteinuria, $^{70-72}$ underscoring the role of the glycocalyx in vascular permeability and permselectivity. The classical model used to describe microvascular fluid exchange is that of Starling, ${ }^{73}$ stating that the fluid filtration rate across the capillary endothelium is determined by the hydraulic and colloid osmotic pressures in the vascular lumen and in the surrounding tissue. This balance has been applied across the entire transendothelial barrier, with the different pressures being assessed globally. The discovery of a relatively thick endothelial glycocalyx and its influence on, e.g., edema formation has led to a major revision of the Starling principle by Weinbaum $^{74}$ and Michel, ${ }^{75}$ who proposed to apply the pressure gradients to the endothelial glycocalyx only. $\mathrm{Hu}$ and Weinbaum used this idea to generate a threedimensional model of permeability over different regions of the endothelial layer, such as the glycocalyx, the endothelial clefts, and the tight junctions. ${ }^{76}$ This model was later simplified into a one-dimensional description of the varying tissue concentration gradients and subsequent permeability of the endothelium. ${ }^{77}$ Another model, known as the glycocalyx-junction-break model applies the Starling mechanism over the glycocalyx and describes its effects on solute and water transport over the endothelium, based on theoretical 'pores' in the endothelium. ${ }^{78}$ Curry has written a review on this model and the influences of phenotypical changes on microvascular permeability. ${ }^{79}$ The importance of the endothelial glycocalyx in controlling extravasation of colloids and fluids is stressed by studies of Rehm et al. ${ }^{80}$ and Jacob et al. ${ }^{81}$, who show impaired endothelial barrier function after glycocalyx degradation in an isolated, perfused heart model. Infusion of $5 \%$ albumin or $6 \%$ hydroxyethyl starch, a natural and an artificial colloid, led to decreased fluid extravasation. However, after 20 mins of warm ischemia, only albumin infusion prevented vascular leakage. This underscores the importance of an intact glycocalyx and the role of plasma-derived proteins for competent glycocalyx functioning. The revised Starling principle has provided more detailed insight into vascular permeability and stresses the importance of the endothelial glycocalyx as a major determinant. ${ }^{82}$ 
Beside its capacity to restrict molecules from reaching the endothelium, the glycocalyx also influences blood cell-vessel wall interactions. It repulses red blood cells from the endothelium. In the microcirculation, a red blood cell exclusion zone flanking the endothelium can be observed in vivo, which is decreased upon lightdye induced breakdown of the glycocalyx. ${ }^{11}$ Similarly, platelets are not often observed interacting with the endothelium in control conditions, whereas partial glycocalyx removal by infusion with oxidized low density lipoprotein (ox-LDL) is accompanied by an increase in platelet-vessel wall interactions. ${ }^{83}$ The role of the glycocalyx in the leukocyte-vessel wall interactions seems dual: on the one hand, it harbors the endothelial cell adhesion molecules, such as P-selectin, ICAM-1, and VCAM-1; on the other hand, it attenuates adhesion of leukocytes to these molecules.

In healthy mouse cremaster muscle venules, breakdown of heparan sulfate side chains through heparitinase leads to an increase in leukocyte adhesion to the endothelium in a dose-dependent manner ${ }^{84}$ Administration of ox-LDL or TNF $\alpha$ also induces leukocyte rolling and adhesion. ${ }^{84,85}$ Steric hindrance seems to play a role in this process. The endothelial glycocalyx is much thicker (ranging from $0.2-0.5 \mu \mathrm{m}$ in capillaries ${ }^{66}$ to $2-3 \mu \mathrm{m}$ in small arteries ${ }^{12}$ and $4.5 \mu \mathrm{m}$ in carotid arteries ${ }^{13}$ ) than the length of the various cell adhesion molecules. P-selectin, for example, the molecule initiating leukocyte rolling, only extends about $38 \mathrm{~nm}$ from the endothelial surface. ${ }^{86}$ On cultured cells transfected with P-selectin constructs with two to six consensus repeats that are consequently shorter than normal P-selectin (nine consensus repeats), the number of attaching neutrophils decreased with decreasing P-selectin length. Cells defective of glycosylation, having a thinner glycocalyx, showed increased attachment of neutrophils. ${ }^{87}$ Hence, in normal conditions, the glycosaminoglycan chains and soluble components of the glycocalyx seem to shield adhesion molecules, thereby, preventing interaction. Stimuli which degrade the glycocalyx or induce a more open mesh, such as enzymes, cytokines, or ischemia and reperfusion, appear to uncover the adhesion molecules, which in turn, allows blood cells to interact with the endothelium. ${ }^{21,83-85}$ One should realize that ligands for endothelial adhesion molecules are not uniformly distributed over the cell membrane of leukocytes, but show association with microvilli and membrane ruffles. ${ }^{88}$ As the glycocalyx is estimated to have a low stiffness, ${ }^{89-91}$ it is likely that the ligand bearing leukocyte membrane extensions protrude relatively easily into the glycocalyx to reach their receptor and enable leukocyte-vessel wall interaction. Furthermore, it has recently been shown that neutrophil recruitment to the endothelium is mediated by myeloperoxidase, which is a cationic enzyme binding to the anionic surfaces of both leukocytes and endothelium, thus facilitating leukocyte 
penetration into the glycocalyx. ${ }^{92}$ In contrast to other tissues, leukocyte-vessel wall interactions occur spontaneously in venules in the skin, even in normal conditions without preceding trauma or inflammation. ${ }^{43,93}$ Whether this is associated with a deviating glycocalyx structure and/or composition in skin microvessels remains to be elucidated.

The presence of a relatively thick endothelial glycocalyx in vivo has great consequences for rheology, especially in the microvasculature. ${ }^{15,94}$ In this part of the circulation, local blood viscosity and hematocrit appear to be modulated by the glycocalyx. Using a physical model, based on hemodynamic and hematocrit measurements in microvascular networks in vivo, Pries and Secomb demonstrated that incorporation of realistic estimates of glycocalyx dimensions in reconstructed mesenteric microvascular networks introduces about a twofold increase in the apparent viscosity of blood. These changes are sufficient to minimize discrepancies between experimentally determined and theoretically predicted microvascular network resistances in previous studies, which were based in previous studies on the apparent viscosity of blood in glycocalyx-devoid glass capillaries. ${ }^{95}$

\section{The endothelial glycocalyx as mechanotransducer}

The endothelium is exposed to mechanical forces induced by blood flow. It has long been recognized that these forces, in particular, shear stress, determine endothelial cell morphology and function. ${ }^{96,97}$ Endothelial cells exposed to shear stress produce nitric oxide (NO) ${ }^{98}$, which is an important determinant of vascular tone. However, the molecule(s) responsible for the translation of biomechanical forces into biochemical signals (mechanotransduction) have not been identified as yet. The glycocalyx has been added to the list of possible candidates.

In studies with cultured endothelial cells, Florian and colleagues ${ }^{99}$ showed that treatment with heparitinase to specifically break down heparan sulfate glycosaminoglycans results in inadequate responses to shear variations and impaired NO production. Similarly, ex vivo experiments on canine femoral arteries conducted by Mochizuki and coworkers exhibited reduced shear-induced NO production after infusion with hyaluronidase, which degrades the hyaluronan glycosaminoglycans in the glycocalyx. ${ }^{100}$ Thus, both heparan sulfate and hyaluronan appear to play a role in detecting and amplifying flow-induced shear forces. ${ }^{100}$

Interestingly, exposure of human umbilical vein endothelial cells to shear stress was found to increase the amount of hyaluronan in the glycocalyx approximately twofold, which may represent a positive feedback for shear stress sensing by endothelial cells. ${ }^{101}$ Furthermore, upregulation of hyaluronan synthase- 2 and increased 
hyaluronan production were mainly seen after exposure to pulsatile shear stress in this model. ${ }^{102}$ Another study showed a correlation between shear stress profile and glycocalyx dimensions in the mouse carotid artery; the laminar flow profiles in the common carotid were found to coincide with a glycocalyx thickness of $399 \pm 174$ $\mathrm{nm}$, whereas disturbed laminar flow in the sinus region of the carotid bifurcation coincided with a thinner glycocalyx of $73 \pm 36 \mathrm{~nm} .{ }^{103}$ Moreover, the flow divider region of the carotid bifurcation, supposedly having an undisturbed high laminar flow profile, was covered with a glycocalyx of $308 \pm 185 \mathrm{~nm}$, comparable to the common carotid glycocalyx thickness.

From these data it seems likely that the glycocalyx plays an important role in mechanotransduction and that its composition is, in turn (at least partly), sheardependent. The different components of the glycocalyx probably operate together, which means that the glycocalyx, as a whole, is responsible for its role as mechanotransducer. This idea is confirmed by theoretical models based on a regular hexagonal distribution of core proteins over the endothelial cell membrane. ${ }^{90}$ Tarbell and Pahakis reviewed concepts on mechanotransduction by the (membranebound) glycocalyx. ${ }^{104}$ They conclude that the glycocalyx core proteins are responsible for transmission of shear stress signals into specific cell signaling processes, e.g., NO production and cytoskeletal reorganization. At the same time, shear stress is transmitted to other regions of the endothelial cell as well, such as intercellular junctions and basal adhesion plaques, which are responsible for additional shear sensing even in the absence of a glycocalyx.

\section{The endothelial glycocalyx as control center for the microenvironment}

The proteoglycans in the glycocalyx contribute greatly to its functional importance. The glycosaminoglycan chain variety arising from chain epimerization, elongation, and most notably, chain sulfation, gives rise to a heterogeneous surface to which a lot of plasma-derived molecules can dock. Table 2.3 lists a number of molecules, which depend on interaction with the glycocalyx for their functionality. Docking of plasma derived molecules can influence the local environment in several ways: (1) Binding of receptors or enzymes and their ligands to the endothelial glycocalyx causes a localized rise in concentration of these substances, which enables proper signaling or enzymatic modification. Fibroblast growth factor (FGF) signaling is mediated in this way and is known to depend completely on the interaction of both ligand and receptor with the glycoclyx. ${ }^{105,106}$ Similarly, the glycocalyx is involved in the lipolytic system, binding both lipoprotein lipase and its ligand low density lipo- 
Table 2.3 Molecules dependent on interaction with the endothelial glycocalyx for proper functioning

\begin{tabular}{llc}
\hline \hline Molecule & Primary function & Ref. \\
\hline \hline $\begin{array}{l}\text { Antithrombin } \\
\text { III }\end{array}$ & $\begin{array}{l}\text { Potent inactivator of procoagulant proteases such as thrombin, } \\
\text { factor Xa and factor IXa; activity enhanced by heparin or } \\
\text { heparan sulfate }\end{array}$ & 112 \\
Heparin & $\begin{array}{l}\text { Inactivator of the procoagulant protease thrombin; activated by } \\
\text { co-factor II }\end{array}$ & 114 \\
TFPI & Anticoagulant protein blocking activated factor VII and X & 116, \\
& & 117 \\
LPL & Enzyme involved in breakdown of LDL & 107 \\
LDL & Transports cholesterol and triglycerides through the circulation & 108 \\
VEGF & $\begin{array}{l}\text { Potent stimulator of angiogenesis, production of which is trig- } \\
\text { gered by hypoxia }\end{array}$ & 151 \\
TGFB1/2 & $\begin{array}{l}\text { Growth factor known to mediate in a lot of signaling pathways, } \\
\text { including SMC differentiation and vascular tone and reactivity }\end{array}$ & 152 \\
FGF(r) & $\begin{array}{l}\text { Growth factor (receptor) involved in endothelial cell prolifera- } \\
\text { tion and angiogenesis }\end{array}$ & 105, \\
ec-SOD & Extracellular quencher of ROS & 106 \\
IL 2 - 5, 7, 8, & $\begin{array}{l}\text { Chemotaxis of leukocytes to the subendothelium; involved in } \\
\text { arrest and diapedesis }\end{array}$ & 121 \\
\hline
\end{tabular}

TFPI tissue factor pathway inhibitor; $L P L$ lipoprotein lipase; $L D L$ low density lipoprotein; VEGF vascular endothelial growth factor; TGFB1/2 transforming growth factor $\beta 1$ or $\beta 2 ; F G F(r)$ fibroblast growth factor (receptor); ec-SOD extracellular superoxide dismutase; IL interleukin; RANTES Regulated on Activation, Normal T Expressed and Secreted - also known as chemokine CCL5; SMC smooth muscle cell; ROS reactive oxygen species

protein (LDL). ${ }^{107,} 108$ (2) Binding of plasma-derived molecules to the glycocalyx can lead to a local concentration gradient, which is often seen in growth factorregulated gene transcription and developmental processes. ${ }^{109,110}$ (3) Docking of enzymes and their agonists or inhibitors to the glycocalyx adds a vasculoprotective role to glycocalyx functionality. Several important anticoagulant mediators can bind to the glycocalyx, such as antithrombin III, heparin co-factor II, thrombomodulin, and tissue factor pathway inhibitor (TFPI). Antithrombin III is a strong inhibitor of procoagulant enzymes like thrombin and activated factors $X$ and IX (FXa and FIXa). ${ }^{111}$ It is known to bind to specific regions in heparan sulfate, which enhances its anticoagulant activity. ${ }^{112}$ Heparin co-factor $\mathrm{II}$ is a thrombin-specific protease inhibitor, ${ }^{113}$ which is activated by dermatan sulfate in the glycocalyx. ${ }^{114}$ Thrombo- 
modulin is a chondroitin sulfate containing protein expressed by endothelial cells and is able to convert thrombin from a procoagulant enzyme to an activator of the protein $C$ pathway, thus, becoming anticoagulant. ${ }^{115}$ TFPI is a potent inhibitor of FVIla and FXa. TFPI is believed to bind to the glycocalyx via heparan sulfates, but other proteins could be involved as well. ${ }^{116}$ Furthermore, uptake and degradation of TFPI-FXa complexes depends on heparan sulfates in the glycocalyx. ${ }^{117}$ All these anticoagulant molecules present in the glycocalyx contribute to the thromboresistant nature of healthy endothelium. ${ }^{118}$ The endothelial glycocalyx also modulates inflammatory responses by binding cytokines and attenuating binding of cytokines to cell surface receptors. Shedding of heparan sulfate from the glycocalyx results in increased endothelial cell sensitivity to activation by cytokines. ${ }^{119,}{ }^{120}$ Another aspect of the vasculoprotective role of the endothelial glycocalyx is its capacity to bind quenchers of oxygen radicals, such as extracellular superoxide dismutase (SOD). ${ }^{121}$ These enzymes help to reduce the oxidative stress and keep up NO bioavailability, thus, preventing the endothelium from becoming dysfunctional.

\section{The endothelial glycocalyx in pathophysiology}

In healthy vessels, the endothelial glycocalyx determines vascular permeability, attenuates blood cell-vessel wall interactions, mediates shear stress sensing, enables balanced signaling, and fulfills a vasculoprotective role. But when it is disrupted or modified, these properties are lost, as has been shown through direct targeting of the glycocalyx in experimental settings. In the last years, evidence is emerging that (damage to) the glycocalyx plays a pivotal role in several vascular pathologies. Here, we will discuss its suspected roles in diabetes, ische$\mathrm{mia} /$ reperfusion, and atherosclerosis.

\section{Diabetes}

Diabetes is a clinically well-known disease with far-reaching complications, such as retino- and nephropathy, and elevated risks for atherothrombotic cardiovascular events. One of the hallmarks of diabetes is insulin absence or resistance and subsequent hyperglycemia, impairing the protective capacity of the vessel wall, ${ }^{122}$ and resulting in enhanced endothelial permeability ${ }^{123}$ and impaired NO synthase function. ${ }^{124}$ However, a common pathway leading to these vascular dysfunctions has not been identified. Recently, it was shown that the systemic glycocalyx volume of healthy volunteers, as assessed by comparing the intravascular distribution volume of a glycocalyx-permeable and a glycocalyx-impermeable tracer, was halved within $6 \mathrm{~h}$ after induction of acute hyperglycemia. ${ }^{125}$ Using the same methodology, the systemic glycocalyx volume in type 1 diabetics was found to be about half of that of 
healthy controls; it was further reduced in diabetics with microalbuminuria. ${ }^{126}$ In the same study, plasma levels of hyaluronan and hyaluronidase were found to be elevated in patients with diabetes, reflecting increased synthesis and shedding of hyaluronan under hyperglycemic conditions. Both studies show that acute and long-standing hyperglycemia is associated with profound reduction of glycocalyx dimensions. Interestingly, treatment of diabetic subjects with sulodexide (a glycocalyx precursor which can be administered orally) attenuated both increased vascular permeability and decreased glycocalyx thickness. ${ }^{127}$ It is tempting to speculate that this damage to the glycocalyx contributes to endothelial dysfunction in hyperglycemic conditions, which can be measured in nondiabetic subjects as well. ${ }^{128}$ Further studies are needed to investigate whether glycocalyx perturbation is responsible for the (micro)vascular complications in diabetes, and whether prolonged treatment with substances like sulodexide is able to decrease long-term cardiovascular risk.

\section{Ischemia/reperfusion}

Damage to tissues during a period of absent or decreased flow (total or partial ischemia) can paradoxically be exaggerated by restoration of blood flow (reperfusion). Although the severity of damage resulting from ischemia/reperfusion varies between tissues, a common component of this pathologic process for all organs is microvascular dysfunction. ${ }^{129,130}$ Endothelial cells play a central role and exhibit swelling and detachment from the basement membrane after ischemia/reperfusion. ${ }^{131}$ Especially in postcapillary venules, endothelial cells suffer from increased oxidative stress, ${ }^{132}$ leukocytes adhere and transmigrate, ${ }^{133,134}$ and vascular permeability increases. ${ }^{135}$ These endothelial consequences of ischemia/reperfusion allude at involvement of the endothelial glycocalyx. Indeed, it was shown by Mulivor and Lipowsky ${ }^{21}$ that intestinal ischemia/reperfusion led to significant reduction of glycocalyx thickness in rat mesenteric venules, most likely due to shedding of glycosaminoglycan chains. The effects of ischemia/reperfusion on the glycocalyx could be attenuated by blockade of xanthine-oxidoreductase, which is an endogenous reactive oxygen species (ROS) producing enzyme bound to heparan sulfate domains in the glycocalyx. This way, a central role for ROS in disruption and shedding of the glycocalyx in ischemia/reperfusion was established. ${ }^{136}$ Infusion of exogenous hyaluronan to restore the glycocalyx or administration of pertussis toxin, which inhibits G-protein-mediated shedding of glycosaminoglycan chains, also reduced ischemia/reperfusion-induced damage. ${ }^{21}$ In a model of perfused isolated guinea pig hearts ischemia/reperfusion led to shedding of glycocalyx components and adhesion of neutrophils to the vascular endothelium. ${ }^{137}$ Protec- 
tion of the glycocalyx with hydrocortisone or antithrombin during the reperfusion period reduced glycocalyx shedding and neutrophil adhesion significantly. ${ }^{137}$ In humans, involvement of the glycocalyx in ischemia/reperfusion is suggested from observations in kidneys used for transplantation; kidneys exposed to prolonged ischemia showed reduced capillary blood flow and endothelial glycocalyx thickness after reperfusion. ${ }^{138}$ Together, these data hint at a role for the endothelial glycocalyx in the pathophysiology of ischemia/reperfusion-induced tissue damage. However, its relative contribution to this process and the impact of possible therapeutical interventions are yet to be established. ${ }^{139-141}$

\section{Atherosclerosis}

Atherosclerosis is a large artery disease and typically requires high plasma levels of LDL to develop at predilection sites ${ }^{142}$ that are marked by disturbed flow profiles. Subendothelial retention of atherogenic lipoproteins and subsequent inflammatory responses lead to the formation of subendothelial plaques. ${ }^{143,}{ }^{144}$ The role of the endothelial glycocalyx in atherogenesis is, as yet, not well established, but there are some interesting observations which point at its involvement.

In 2000, Vink and colleagues ${ }^{83}$ showed that administration of clinically relevant doses of ox-LDL leads to a disruption of the glycocalyx in hamster cremaster muscle microcirculation and evokes local platelet adhesion. Co-infusion with SOD and catalase, enzymes catalyzing the dismutation of superoxide anion and the decomposition of hydrogen peroxide, abolished this effect of ox-LDL, implicating a role for oxygen-derived free radicals. Indeed, loss of glycocalyx results in shedding of endogenous protective enzymes, such as extracellular SOD, and increases the oxidative stress on endothelial cells. This was further illustrated by a recent study by van den Berg et al., ${ }^{103}$ showing a reduction in glycocalyx dimensions of the mouse carotid artery due to a high-fat, high-cholesterol diet. Furthermore, an inverse relation between glycocalyx thickness and intima-media ratio was found, reflecting a reduction of vasculoprotective capacity of the endothelial glycocalyx at sites with higher atherogenic risk. In healthy mice, regional variations were found showing a thinner glycocalyx in the internal carotid sinus region compared to the common carotid artery. Moreover, this thinner glycocalyx was associated with increased leakage of LDL through the glycocalyx. ${ }^{63}$ Recently, Nagy et al. ${ }^{145}$ reported on the effect of prolonged inhibition of hyaluronan synthesis in atherogenic mice. These mice showed a marked increase in aortic plaque burden, increased numbers of plaque macrophages and a prothrombotic state, while the endothelial glycocalyx thickness was severely reduced. Together, these data suggest that the endothelial 
glycocalyx is involved in the initiation and progression of the atherosclerotic process. $^{3}$

In summary, the endothelial glycocalyx appears to be perturbed in several vascular diseases. It remains to be elucidated whether glycocalyx perturbation is causally involved in the pathophysiology of these diseases. If so, restoration of the glycocalyx may be a therapeutic target of interest. Furthermore, identification of specific glycosaminoglycan domains involved in these diseases, as a platform for other substances or signaling pathways, might also prove to be of therapeutic value. ${ }^{4}$

\section{Visualization techniques}

Because of the functional importance of the endothelial glycocalyx, development of direct visualization techniques is crucial to establish its exact role. The glycocalyx can be labeled by administration of specific markers that attach to one or more of its components, making them fluorescent or detectable. Preparation of (parts of) the vessel would then allow specific microscopic imaging of the endothelial glycocalyx. Regretfully, the glycocalyx is very vulnerable and easily disturbed or dehydrated during vessel handling and preparation protocols. As a result, glycocalyx dimensions are easily underestimated, which is illustrated by the first images of the glycocalyx, made by transmission electron microscopy (TEM) in 1966 with the use of the probe ruthenium red; glycocalyx thickness measured this way approximated $20 \mathrm{~nm}$ in capillaries. ${ }^{1}$ Since then, many other attempts were made to image the glycocalyx using TEM. On bovine aorta endothelial cells under shear stress condi-

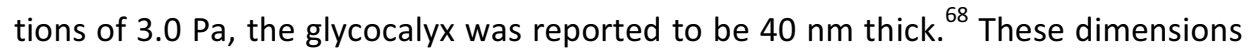
did not comply with theoretical estimates predicting the glycocalyx to be up to 1 $\mu \mathrm{m}$ thick. ${ }^{6}$ Using a new staining protocol with Alcian blue $8 \mathrm{GX}$, van den Berg et al. ${ }^{66}$ applied TEM to measure endothelial glycocalyx dimensions in rat myocardial capillaries, which revealed that endothelial cells are covered by a 200- to 500-nm thick glycocalyx (Figure 2.2a). Hyaluronidase treatment before fixation and staining resulted in significant reduction of this layer to $100-200 \mathrm{~nm}$. The groups of Haraldsson $^{146}$ and of Rostgaard and Qvortrup ${ }^{147,}{ }^{148}$ improved the TEM staining protocol using fluorocarbon-based oxygen carrying fixatives, revealing glycocalyces as thick as $60-200 \mathrm{~nm}$ in glomerular capillaries, and $50-100 \mathrm{~nm}$ in intestinal fenestrated capillaries. Apparently, the new staining and preparation protocols improved glycocalyx conservation in TEM experiments. However, TEM cannot be used in the in vivo situation.

Some 30 years after the first TEM images were made, Vink et al. ${ }^{11}$ used intravital microscopy to visualize the endothelial glycocalyx in hamster cremaster muscle 
capillaries in vivo using indirect approaches. The glycocalyx was recognized as a red blood cell 'exclusion zone' or 'gap' between the flowing red blood cells and the endothelium. In addition, the plasma was labeled by a fluorescent dextran, and the glycocalyx then appeared as a plasma exclusion zone (Figure 2.2b). Interestingly, no exclusion zone was found for rolling white blood cells, suggesting that they have the ability to compress the glycocalyx in these vessels, which complies with the estimated low stiffness of the glycocalyx. ${ }^{89-91}$ Substraction of the diameter of the plasma column from the anatomical internal diameter revealed the dimensions of the glycocalyx, which appeared to be $0.4-0.5 \mu \mathrm{m}$ thick. ${ }^{11}$ This method has been used in many studies since, primarily in the cremaster muscle microcirculation of hamsters ${ }^{64,65,85}$ or mice. ${ }^{84,136}$ This tissue is suited for intravital microscopy because it is thin and translucent, allowing clear visualization of microvascular endothelial cells and flowing blood cells, with low or absent vessel wall motion (Figure 2.2b). In addition, local flow velocities can be measured. However, the estimation of the glycocalyx thickness using intravital microscopy-based methods is indirect. Furthermore, intravital microscopy cannot be applied to image the endothelial glycocalyx in larger vessels.

Direct visualization of the glycocalyx has been performed via several approaches, mostly using lectins which are proteins that bind specific disaccharide moieties of glycosaminoglycan chains. ${ }^{21,99,149}$ Other labels include antibodies for heparan sulfate, syndecan-1 or hyaluronan. ${ }^{21,63,99}$ When attaching these markers to a fluorescent probe, advanced microscopic techniques can be applied to visualize the glycocalyx. Confocal laser scanning microscopy (CLSM) enables optical sectioning with good optical resolution, allowing three-dimensional reconstructions of the specimen. Lectin labeling of the glycocalyx of cultured human umbilical vein endothelial cells and subsequent CLSM imaging revealed a surface layer as thick as $2.5 \pm 0.5$ $\mu \mathrm{m} .{ }^{149} \mathrm{CLSM}$ has also been used to detect concentration changes of fluorescently labeled lectins in the glycocalyx of fixated rat mesentery postcapillary venules in case of ischemia/reperfusion and inflammation. ${ }^{21}$ Because larger vessels have thicker walls, which results in lower light penetration depths with significant loss of resolution at higher depths $(>40 \mu \mathrm{m})^{150}$ due to increased scattering of signal, CLSM is less suitable for imaging of the glycocalyx in arteries, unless they are cut open to access the glycocalyx. ${ }^{63}$

A promising technique to directly visualize the glycocalyx in larger vessels, both ex vivo and in vivo, is two-photon laser scanning microscopy (TPLSM). TPLSM depends 


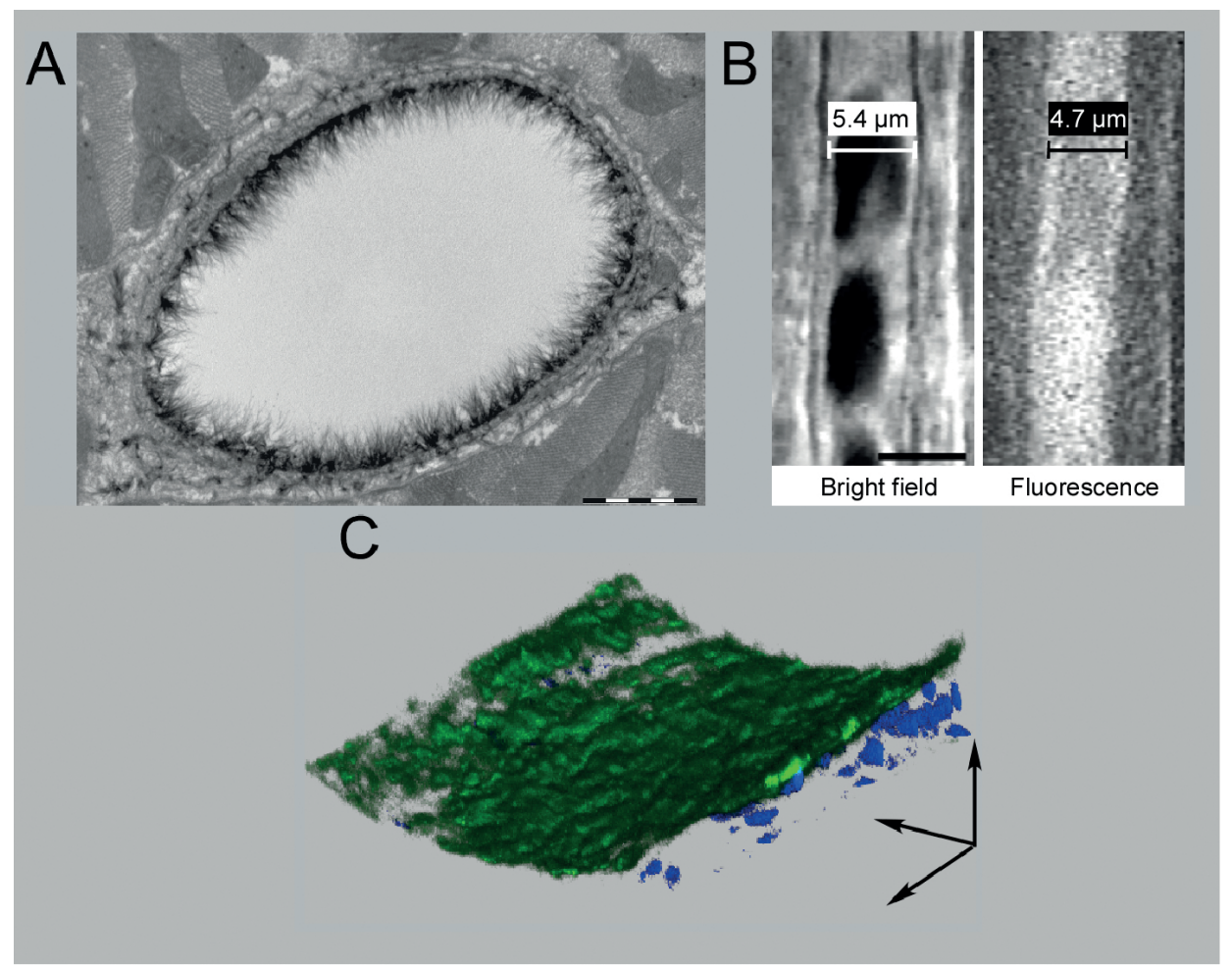

Figure 2.2 Visualization of the endothelial glycocalyx with different microscopic techniques.

$a$ Endothelial glycocalyx of a rat left ventricular myocardial capillary stained with Alcian blue 8GX and visualized using electron microscopy. Bar represents $1 \mu \mathrm{m}$. Reproduced with permission from reference number 66.

$b$ Intravital microscopic recording of the endothelial glycocalyx of a hamster cremaster muscle capillary. The anatomical diameter of $5.4 \mu \mathrm{m}$ is larger than the red blood cell column width (left pane) or the plasma column width (right pane) labeled with fluorescent dextran (70 kDa). This difference is caused by the presence of the endothelial glycocalyx. The bar in the left pane represents $5 \mu \mathrm{m}$. Reproduced with permission from reference number 11.

$c$ Endothelial glycocalyx of a mouse common carotid artery. Three-dimensional reconstruction of a series of optical slices obtained with two-photon laser scanning microscopy showing part of the vessel wall. The intact vessel was perfused with FITC-labeled lectin (WGA) to stain the endothelial glycocalyx (green) and SYTO 41 to label cell nuclei (blue). The arrows indicate the direction of the X, Y, and Z-axis. The scanned volume approximates $200 \times 200 \times 60 \mu \mathrm{m}^{3}$. For details on methodology see also reference number 13 .

on excitation of a fluorophore by simultaneous uptake (i.e., within $10^{-18} \mathrm{~s}$ ) of two red photons, instead of one blue photon as in conventional fluorescence excitation. The use of long wavelength red photons reduces scattering, and hence, increases penetration depth into tissue. Excitation of the fluorophore and consequent fluorescence only occurs at the focal point of the illumination cone, as the probability of two-photon excitation depends on the squared intensity of the excitatory pho- 
tons. Any light received by the photomultipliers has to originate from the focal position, so scattering of the emitted photons does not influence resolution and no pinholes are required. As a consequence, TPLSM offers good resolution and optical sectioning at reasonable acquisition speed, while bleaching and phototoxicity of the dyes is limited to the focal position. The combination of enhanced penetration depth, good resolution, optical sectioning, and low phototoxicity makes TPLSM a suitable technique to visualize the delicate endothelial glycocalyx in larger vessels. This idea was confirmed by a pilot study by Megens and colleagues ${ }^{13}$ in which the endothelial glycocalyx was imaged with TPLSM in intact mouse carotid arteries (Figure 2.2c); the glycocalyx thickness was found to be $4.5 \pm 1.0 \mu \mathrm{m}$. As TPLSM is also applicable in vivo in rodents, ${ }^{150}$ it might prove to be a good approach of in vivo visualization of the glycocalyx in the macrocirculation of these animals.

\section{Conclusions}

Overlying the vascular endothelium, the glycocalyx is a membrane-bound mesh in which plasma-derived molecules integrate. It exerts a variety of functions, important in normal vascular physiology and also in vascular disease. Although data from experiments in microcirculation, and more recently, in macrocirculation strongly suggests a vasculoprotective role for the glycocalyx, research on this subject is hampered by lack of a good visualization technique. Two-photon laser scanning microscopy may prove to be a successful tool in achieving direct visualization of the glycocalyx in larger arteries in rodents, both ex vivo and in vivo, with the possibility to analyze focal variations in the composition or integrity of this layer.

\section{Acknowledgements}

The authors thank Remco Megens for his support in the visualization of the glycocalyx with two-photon microscopy and Bernard van den Berg for providing his EMpicture. This research is supported by NWO grant \#902-16-276 and SenterNovem (BSIK 03033). 


\section{References}

1. Luft JH. Fine structures of capillary and endocapillary layer as revealed by ruthenium red. Fed Proc. 1966;25:1773-1783.

2. Pries AR, Secomb TW, Gaehtgens P. The endothelial surface layer. Pflugers Arch. 2000;440:653666.

3. Nieuwdorp M, Meuwese MC, Vink H, Hoekstra JB, Kastelein JJ, Stroes ES. The endothelial glycocalyx: a potential barrier between health and vascular disease. Curr Opin Lipidol. 2005;16:507-511.

4. Ballinger ML, Nigro J, Frontanilla KV, Dart AM, Little PJ. Regulation of glycosaminoglycan structure and atherogenesis. Cell Mol Life Sci. 2004;61:1296-1306.

5. Klitzman B, Johnson PC. Capillary network geometry and red cell distribution in hamster cremaster muscle. Am J Physiol. 1982;242:H211-219.

6. Klitzman B, Duling BR. Microvascular hematocrit and red cell flow in resting and contracting striated muscle. Am J Physiol. 1979;237:H481-490.

7. Sarelius IH, Duling BR. Direct measurement of microvessel hematocrit, red cell flux, velocity, and transit time. Am J Physiol. 1982;243:H1018-1026.

8. Lipowsky HH, Kovalcheck S, Zweifach BW. The distribution of blood rheological parameters in the microvasculature of cat mesentery. Circ Res. 1978;43:738-749.

9. Pries AR, Ley K, Gaehtgens P. Generalization of the Fahraeus principle for microvessel networks. Am J Physiol. 1986;251:H1324-1332.

10. Desjardins C, Duling BR. Heparinase treatment suggests a role for the endothelial cell glycocalyx in regulation of capillary hematocrit. Am J Physiol. 1990;258:H647-654.

11. Vink H, Duling BR. Identification of distinct luminal domains for macromolecules, erythrocytes, and leukocytes within mammalian capillaries. Circ Res. 1996;79:581-589.

12. van Haaren PM, VanBavel E, Vink H, Spaan JA. Localization of the permeability barrier to solutes in isolated arteries by confocal microscopy. Am J Physiol Heart Circ Physiol. 2003;285:H2848-2856.

13. Megens RT, Reitsma S, Schiffers PH, Hilgers RH, De Mey JG, Slaaf DW, oude Egbrink MG, van Zandvoort MA. Two-photon microscopy of vital murine elastic and muscular arteries. Combined structural and functional imaging with subcellular resolution. J Vasc Res. 2007;44:87-98.

14. Ghitescu L, Robert M. Diversity in unity: the biochemical composition of the endothelial cell surface varies between the vascular beds. Microsc Res Tech. 2002;57:381-389.

15. Lipowsky HH. Microvascular rheology and hemodynamics. Microcirculation. 2005;12:5-15.

16. Fransson LA, Belting M, Cheng F, Jonsson M, Mani K, Sandgren S. Novel aspects of glypican glycobiology. Cell Mol Life Sci. 2004;61:1016-1024.

17. Carey DJ. Syndecans: multifunctional cell-surface co-receptors. Biochem J. 1997;327 (Pt 1):1-16.

18. Iozzo RV. Perlecan: a gem of a proteoglycan. Matrix Biol. 1994;14:203-208.

19. Kinsella MG, Bressler SL, Wight TN. The regulated synthesis of versican, decorin, and biglycan: extracellular matrix proteoglycans that influence cellular phenotype. Crit Rev Eukaryot Gene Expr. 2004;14:203-234.

20. Rapraeger A. Transforming growth factor (type beta) promotes the addition of chondroitin sulfate chains to the cell surface proteoglycan (syndecan) of mouse mammary epithelia. I Cell Biol. 1989;109:2509-2518.

21. Mulivor AW, Lipowsky HH. Inflammation- and ischemia-induced shedding of venular glycocalyx. Am J Physiol Heart Circ Physiol. 2004;286:H1672-1680.

22. Esko JD, Selleck SB. Order out of chaos: assembly of ligand binding sites in heparan sulfate. Annu Rev Biochem. 2002;71:435-471.

23. Sugahara K, Mikami T, Uyama T, Mizuguchi S, Nomura K, Kitagawa H. Recent advances in the structural biology of chondroitin sulfate and dermatan sulfate. Curr Opin Struct Biol. 2003;13:612-620. 
24. Funderburgh JL. Keratan sulfate: structure, biosynthesis, and function. Glycobiology. 2000;10:951958.

25. Laurent TC, Fraser JR. Hyaluronan. Faseb J. 1992;6:2397-2404.

26. Lee JY, Spicer AP. Hyaluronan: a multifunctional, megaDalton, stealth molecule. Curr Opin Cell Biol. 2000;12:581-586.

27. Ihrcke NS, Wrenshall LE, Lindman BJ, Platt JL. Role of heparan sulfate in immune system-blood vessel interactions. Immunol Today. 1993;14:500-505.

28. Tkachenko E, Rhodes JM, Simons M. Syndecans: new kids on the signaling block. Circ Res. 2005;96:488-500.

29. Rapraeger A, Jalkanen $M$, Endo E, Koda J, Bernfield $M$. The cell surface proteoglycan from mouse mammary epithelial cells bears chondroitin sulfate and heparan sulfate glycosaminoglycans. J Biol Chem. 1985;260:11046-11052.

30. Nandi A, Estess P, Siegelman MH. Hyaluronan anchoring and regulation on the surface of vascular endothelial cells is mediated through the functionally active form of CD44. I Biol Chem. 2000;275:14939-14948.

31. Weigel PH, Hascall VC, Tammi M. Hyaluronan synthases. J Biol Chem. 1997;272:13997-14000.

32. Grammatikakis N, Grammatikakis A, Yoneda M, Yu Q, Banerjee SD, Toole BP. A novel glycosaminoglycan-binding protein is the vertebrate homologue of the cell cycle control protein, Cdc37. J Biol Chem. 1995;270:16198-16205.

33. Das S, Deb TB, Kumar R, Datta K. Multifunctional activities of human fibroblast 34-kDa hyaluronic acid-binding protein. Gene. 1997;190:223-225.

34. Evanko SP, Wight TN. Intracellular localization of hyaluronan in proliferating cells. J Histochem Cytochem. 1999;47:1331-1342.

35. Vogl-Willis CA, Edwards IJ. High-glucose-induced structural changes in the heparan sulfate proteoglycan, perlecan, of cultured human aortic endothelial cells. Biochim Biophys Acta. 2004;1672:36-45.

36. Kansas GS, Saunders KB, Ley K, Zakrzewicz A, Gibson RM, Furie BC, Furie B, Tedder TF. A role for the epidermal growth factor-like domain of $\mathrm{P}$-selectin in ligand recognition and cell adhesion. $J$ Cell Biol. 1994;124:609-618.

37. Gibson RM, Kansas GS, Tedder TF, Furie B, Furie BC. Lectin and epidermal growth factor domains of P-selectin at physiologic density are the recognition unit for leukocyte binding. Blood. 1995;85:151158.

38. Sperandio M. Selectins and glycosyltransferases in leukocyte rolling in vivo. Febs J. 2006;273:43774389.

39. Koedam JA, Cramer EM, Briend E, Furie B, Furie BC, Wagner DD. P-selectin, a granule membrane protein of platelets and endothelial cells, follows the regulated secretory pathway in AtT-20 cells. J Cell Biol. 1992;116:617-625.

40. Dole VS, Bergmeier W, Mitchell HA, Eichenberger SC, Wagner DD. Activated platelets induce Weibel-Palade-body secretion and leukocyte rolling in vivo: role of P-selectin. Blood. 2005;106:2334-2339.

41. Sperandio M, Ley K. The Physiology and Pathophysiology of P-selectin. Mod. Asp. Immunobiol. 2005;15:24-26.

42. Jung U, Ley K. Regulation of E-selectin, P-selectin, and intercellular adhesion molecule 1 expression in mouse cremaster muscle vasculature. Microcirculation. 1997;4:311-319.

43. oude Egbrink MG, Janssen GH, Ookawa K, Slaaf DW, Reneman RS, Wehrens XH, Maaijwee KJ, Ohshima N, Struijker Boudier HA, Tangelder GJ. Especially polymorphonuclear leukocytes, but also monomorphonuclear leukocytes, roll spontaneously in venules of intact rat skin: involvement of Eselectin. J Invest Dermatol. 2002;118:323-326. 
44. Weninger W, Ulfman LH, Cheng G, Souchkova N, Quackenbush EJ, Lowe JB, von Andrian UH. Specialized contributions by alpha(1,3)-fucosyltransferase-IV and FucT-VII during leukocyte rolling in dermal microvessels. Immunity. 2000;12:665-676.

45. Xiong JP, Stehle T, Goodman SL, Arnaout MA. Integrins, cations and ligands: making the connection. J Thromb Haemost. 2003;1:1642-1654.

46. Bombeli T, Schwartz BR, Harlan JM. Adhesion of activated platelets to endothelial cells: evidence for a GPIIbIlla-dependent bridging mechanism and novel roles for endothelial intercellular adhesion molecule 1 (ICAM-1), alphavbeta3 integrin, and GPIbalpha. J Exp Med. 1998;187:329-339.

47. Ruegg C, Mariotti A. Vascular integrins: pleiotropic adhesion and signaling molecules in vascular homeostasis and angiogenesis. Cell Mol Life Sci. 2003;60:1135-1157.

48. Muller AM, Hermanns MI, Cronen C, Kirkpatrick CJ. Comparative study of adhesion molecule expression in cultured human macro- and microvascular endothelial cells. Exp Mol Pathol. 2002;73:171-180.

49. Stewart RJ, Kashour TS, Marsden PA. Vascular endothelial platelet endothelial adhesion molecule-1 (PECAM-1) expression is decreased by TNF-alpha and IFN-gamma. Evidence for cytokine-induced destabilization of messenger ribonucleic acid transcripts in bovine endothelial cells. J Immunol. 1996;156:1221-1228.

50. Huang MT, Mason JC, Birdsey GM, Amsellem V, Gerwin N, Haskard DO, Ridley AJ, Randi AM. Endothelial intercellular adhesion molecule (ICAM)-2 regulates angiogenesis. Blood. 2005;106:16361643.

51. Lopez JA. The platelet glycoprotein Ib-IX complex. Blood Coagul Fibrinolysis. 1994;5:97-119.

52. Ruggeri ZM. Von Willebrand factor, platelets and endothelial cell interactions. J Thromb Haemost. 2003;1:1335-1342.

53. Berndt MC, Shen Y, Dopheide SM, Gardiner EE, Andrews RK. The vascular biology of the glycoprotein Ib-IX-V complex. Thromb Haemost. 2001;86:178-188.

54. Wu G, Essex DW, Meloni FJ, Takafuta T, Fujimura K, Konkle BA, Shapiro SS. Human endothelial cells in culture and in vivo express on their surface all four components of the glycoprotein $\mathrm{Ib} / \mathrm{IX} / \mathrm{V}$ complex. Blood. 1997;90:2660-2669.

55. Tan L, Kowalska MA, Romo GM, Lopez JA, Darzynkiewicz Z, Niewiarowski S. Identification and characterization of endothelial glycoprotein lb using viper venom proteins modulating cell adhesion. Blood. 1999;93:2605-2616.

56. Huxley VH, Curry FE. Differential actions of albumin and plasma on capillary solute permeability. Am J Physiol. 1991;260:H1645-1654.

57. Kresse $H$, Hausser $H$, Schonherr E, Bittner K. Biosynthesis and interactions of small chondroitin/dermatan sulphate proteoglycans. Eur J Clin Chem Clin Biochem. 1994;32:259-264.

58. Raman R, Sasisekharan V, Sasisekharan R. Structural insights into biological roles of proteinglycosaminoglycan interactions. Chem Biol. 2005;12:267-277.

59. Zhuo L, Hascall VC, Kimata K. Inter-alpha-trypsin inhibitor, a covalent protein-glycosaminoglycanprotein complex. J Biol Chem. 2004;279:38079-38082.

60. Scott JE, Thomlinson AM, Prehm P. Supramolecular organization in streptococcal pericellular capsules is based on hyaluronan tertiary structures. Exp Cell Res. 2003;285:1-8.

61. Scott JE, Heatley F. Hyaluronan forms specific stable tertiary structures in aqueous solution: a $13 \mathrm{C}$ NMR study. Proc Natl Acad Sci U S A. 1999;96:4850-4855.

62. Tarbell JM, Weinbaum S, Kamm RD. Cellular fluid mechanics and mechanotransduction. Ann Biomed Eng. 2005;33:1719-1723.

63. van den Berg BM, Spaan JA, Vink H. Impaired glycocalyx barrier properties contribute to enhanced intimal low-density lipoprotein accumulation at the carotid artery bifurcation in mice. Pflugers Arch. 2009;457:1199-1206. 
64. Vink H, Duling BR. Capillary endothelial surface layer selectively reduces plasma solute distribution volume. Am J Physiol Heart Circ Physiol. 2000;278:H285-289.

65. Henry $C B$, Duling BR. Permeation of the luminal capillary glycocalyx is determined by hyaluronan. Am J Physiol. 1999;277:H508-514.

66. van den Berg BM, Vink $\mathrm{H}$, Spaan JA. The endothelial glycocalyx protects against myocardial edema. Circ Res. 2003;92:592-594.

67. Henry CB, Duran WN, DeFouw DO. Permselectivity of angiogenic microvessels following alteration of the endothelial fiber matrix by oligosaccharides. Microvasc Res. 1997;53:150-155.

68. Ueda A, Shimomura M, Ikeda M, Yamaguchi R, Tanishita K. Effect of glycocalyx on shear-dependent albumin uptake in endothelial cells. Am J Physiol Heart Circ Physiol. 2004;287:H2287-2294.

69. van Haaren PM, VanBavel E, Vink H, Spaan JA. Charge modification of the endothelial surface layer modulates the permeability barrier of isolated rat mesenteric small arteries. Am J Physiol Heart Circ Physiol. 2005;289:H2503-2507.

70. Meuwese MC, Broekhuizen LN, Kuikhoven M, Heeneman S, Lutgens E, Gijbels MJ, Nieuwdorp M, Peutz CJ, Stroes ES, Vink H, van den Berg BM. Endothelial surface layer degradation by chronic hyaluronidase infusion induces proteinuria in apolipoprotein E-deficient mice. PLOS One. 2010;5:e14262.

71. Jeansson M, Bjorck K, Tenstad O, Haraldsson B. Adriamycin alters glomerular endothelium to induce proteinuria. J Am Soc Nephrol. 2009;20:114-122.

72. Friden V, Oveland E, Tenstad O, Ebefors K, Nystrom J, Nilsson UA, Haraldsson B. The glomerular endothelial cell coat is essential for glomerular filtration. Kidney Int. 2011;79:1322-1330.

73. Starling EH. On the Absorption of Fluids from the Connective Tissue Spaces. J Physiol. 1896;19:312326.

74. Weinbaum S. 1997 Whitaker Distinguished Lecture: Models to solve mysteries in biomechanics at the cellular level; a new view of fiber matrix layers. Ann Biomed Eng. 1998;26:627-643.

75. Michel CC. Starling: the formulation of his hypothesis of microvascular fluid exchange and its significance after 100 years. Exp Physiol. 1997;82:1-30.

76. Hu X, Weinbaum S. A new view of Starling's hypothesis at the microstructural level. Microvasc Res. 1999;58:281-304.

77. Zhang X, Adamson RH, Curry FR, Weinbaum S. A 1-D model to explore the effects of tissue loading and tissue concentration gradients in the revised Starling principle. Am J Physiol Heart Circ Physiol. 2006;291:H2950-2964.

78. Pappenheimer JR, Renkin EM, Borrero LM. Filtration, diffusion and molecular sieving through peripheral capillary membranes; a contribution to the pore theory of capillary permeability. Am J Physiol. 1951;167:13-46.

79. Curry FR. Microvascular solute and water transport. Microcirculation. 2005;12:17-31.

80. Rehm M, Zahler S, Lotsch M, Welsch U, Conzen P, Jacob M, Becker BF. Endothelial glycocalyx as an additional barrier determining extravasation of $6 \%$ hydroxyethyl starch or $5 \%$ albumin solutions in the coronary vascular bed. Anesthesiology. 2004;100:1211-1223.

81. Jacob M, Bruegger D, Rehm M, Welsch U, Conzen P, Becker BF. Contrasting effects of colloid and crystalloid resuscitation fluids on cardiac vascular permeability. Anesthesiology. 2006;104:12231231.

82. Levick JR, Michel CC. Microvascular fluid exchange and the revised Starling principle. Cardiovasc Res. 2010;87:198-210.

83. Vink $\mathrm{H}$, Constantinescu AA, Spaan JA. Oxidized lipoproteins degrade the endothelial surface layer: implications for platelet-endothelial cell adhesion. Circulation. 2000;101:1500-1502.

84. Constantinescu AA, Vink H, Spaan JA. Endothelial cell glycocalyx modulates immobilization of leukocytes at the endothelial surface. Arterioscler Thromb Vasc Biol. 2003;23:1541-1547. 
85. Henry CB, Duling BR. TNF-alpha increases entry of macromolecules into luminal endothelial cell glycocalyx. Am J Physiol Heart Circ Physiol. 2000;279:H2815-2823.

86. Springer TA. Adhesion receptors of the immune system. Nature. 1990;346:425-434.

87. Patel KD, Nollert MU, McEver RP. P-selectin must extend a sufficient length from the plasma membrane to mediate rolling of neutrophils. J Cell Biol. 1995;131:1893-1902.

88. Sasaki K, Okouchi Y, Rothkotter HJ, Pabst R. Three-dimensional distribution of intercellular adhesion molecule-1 on lymphocytes in the high endothelial venule analyzed by backscatter electron imaging. Acta Anat (Basel). 1998;162:33-39.

89. Secomb TW, Hsu R, Pries AR. A model for red blood cell motion in glycocalyx-lined capillaries. Am J Physiol. 1998;274:H1016-1022.

90. Weinbaum S, Zhang X, Han Y, Vink H, Cowin SC. Mechanotransduction and flow across the endothelial glycocalyx. Proc Natl Acad Sci U S A. 2003;100:7988-7995.

91. Han YF, Weinbaum S, Spaan JAE, Vink H. Large-deformation analysis of the elastic recoil of fibre layers in a Brinkman medium with application to the endothelial glycocalyx. Journal of Fluid Mechanics. 2006;554:217-235.

92. Klinke A, Nussbaum C, Kubala L, Friedrichs K, Rudolph TK, Rudolph V, Paust HJ, Schroder C, Benten D, Lau D, Szocs K, Furtmuller PG, Heeringa P, Sydow K, Duchstein HJ, Ehmke H, Schumacher U, Meinertz T, Sperandio M, Baldus S. Myeloperoxidase attracts neutrophils by physical forces. Blood. 2011;117:1350-1358.

93. Janssen GH, Tangelder GJ, Oude Egbrink MG, Reneman RS. Spontaneous leukocyte rolling in venules in untraumatized skin of conscious and anesthetized animals. Am J Physiol. 1994;267:H1199-1204.

94. Pries AR, Secomb TW. Rheology of the microcirculation. Clin Hemorheol Microcirc. 2003;29:143148.

95. Pries AR, Secomb TW. Microvascular blood viscosity in vivo and the endothelial surface layer. Am J Physiol Heart Circ Physiol. 2005;289:H2657-2664.

96. Dewey CF, Jr., Bussolari SR, Gimbrone MA, Jr., Davies PF. The dynamic response of vascular endothelial cells to fluid shear stress. J Biomech Eng. 1981;103:177-185.

97. Davies PF. Flow-mediated endothelial mechanotransduction. Physiol Rev. 1995;75:519-560.

98. Rubanyi GM, Romero JC, Vanhoutte PM. Flow-induced release of endothelium-derived relaxing factor. Am J Physiol. 1986;250:H1145-1149.

99. Florian JA, Kosky JR, Ainslie K, Pang Z, Dull RO, Tarbell JM. Heparan sulfate proteoglycan is a mechanosensor on endothelial cells. Circ Res. 2003;93:e136-142.

100. Mochizuki S, Vink H, Hiramatsu O, Kajita T, Shigeto F, Spaan JA, Kajiya F. Role of hyaluronic acid glycosaminoglycans in shear-induced endothelium-derived nitric oxide release. Am J Physiol Heart Circ Physiol. 2003;285:H722-726.

101. Gouverneur M, Spaan JA, Pannekoek H, Fontijn RD, Vink H. Fluid shear stress stimulates incorporation of hyaluronan into endothelial cell glycocalyx. Am J Physiol Heart Circ Physiol. 2006;290:H458452.

102. Maroski J, Vorderwuelbecke B, Fiedorowicz K, Da Silva-Azevedo L, Siegel G, Marki A, Pries A, Zakrzewicz $A$. Shear stress increases endothelial hyaluronan synthase 2 and hyaluronan synthesis especially in regard to an atheroprotective flow profile. Exp Physiol. 2011;96:977-986.

103. van den Berg BM, Spaan JA, Rolf TM, Vink H. Atherogenic region and diet diminish glycocalyx dimension and increase intima-to-media ratios at murine carotid artery bifurcation. Am J Physiol Heart Circ Physiol. 2006;290:H915-920.

104. Tarbell JM, Pahakis MY. Mechanotransduction and the glycocalyx. J Intern Med. 2006;259:339-350.

105. Allen BL, Filla MS, Rapraeger AC. Role of heparan sulfate as a tissue-specific regulator of FGF-4 and FGF receptor recognition. J Cell Biol. 2001;155:845-858. 
106. Fromm JR, Hileman RE, Weiler JM, Linhardt RJ. Interaction of fibroblast growth factor-1 and related peptides with heparan sulfate and its oligosaccharides. Arch Biochem Biophys. 1997;346:252-262.

107. Wang CS, Hartsuck J, McConathy WJ. Structure and functional properties of lipoprotein lipase. Biochim Biophys Acta. 1992;1123:1-17.

108. Wilsie LC, Orlando RA. The low density lipoprotein receptor-related protein complexes with cell surface heparan sulfate proteoglycans to regulate proteoglycan-mediated lipoprotein catabolism. $J$ Biol Chem. 2003;278:15758-15764.

109. Perrimon N, Bernfield M. Specificities of heparan sulphate proteoglycans in developmental processes. Nature. 2000;404:725-728.

110. Lin X. Functions of heparan sulfate proteoglycans in cell signaling during development. Development. 2004;131:6009-6021.

111. Quinsey NS, Greedy AL, Bottomley SP, Whisstock JC, Pike RN. Antithrombin: in control of coagulation. Int J Biochem Cell Biol. 2004;36:386-389.

112. Shimada K, Kobayashi M, Kimura S, Nishinaga M, Takeuchi K, Ozawa T. Anticoagulant heparin-like glycosaminoglycans on endothelial cell surface. Jpn Circ J. 1991;55:1016-1021.

113. Parker KA, Tollefsen DM. The protease specificity of heparin cofactor II. Inhibition of thrombin generated during coagulation. J Biol Chem. 1985;260:3501-3505.

114. Tovar AM, de Mattos DA, Stelling MP, Sarcinelli-Luz BS, Nazareth RA, Mourao PA. Dermatan sulfate is the predominant antithrombotic glycosaminoglycan in vessel walls: implications for a possible physiological function of heparin cofactor II. Biochim Biophys Acta. 2005;1740:45-53.

115. Weiler H, Isermann BH. Thrombomodulin. J Thromb Haemost. 2003;1:1515-1524.

116. Kato $\mathrm{H}$. Regulation of functions of vascular wall cells by tissue factor pathway inhibitor: basic and clinical aspects. Arterioscler Thromb Vasc Biol. 2002;22:539-548.

117. Ho G, Broze GJ, Jr., Schwartz AL. Role of heparan sulfate proteoglycans in the uptake and degradation of tissue factor pathway inhibitor-coagulation factor Xa complexes. J Biol Chem. 1997;272:16838-16844.

118. oude Egbrink MG, Van Gestel MA, Broeders MA, Tangelder GJ, Heemskerk JM, Reneman RS, Slaaf DW. Regulation of microvascular thromboembolism in vivo. Microcirculation. 2005;12:287-300.

119. Bode L, Murch S, Freeze HH. Heparan sulfate plays a central role in a dynamic in vitro model of protein-losing enteropathy. J Biol Chem. 2006;281:7809-7815.

120. Bode L, Eklund EA, Murch S, Freeze HH. Heparan sulfate depletion amplifies TNF-alpha-induced protein leakage in an in vitro model of protein-losing enteropathy. Am J Physiol Gastrointest Liver Physiol. 2005;288:G1015-1023.

121. Li Q, Bolli R, Qiu Y, Tang XL, Murphree SS, French BA. Gene therapy with extracellular superoxide dismutase attenuates myocardial stunning in conscious rabbits. Circulation. 1998;98:1438-1448.

122. Nathan DM, Lachin J, Cleary P, Orchard T, Brillon DJ, Backlund JY, O'Leary DH, Genuth S. Intensive diabetes therapy and carotid intima-media thickness in type 1 diabetes mellitus. $N$ Engl J Med. 2003;348:2294-2303.

123. Algenstaedt $P$, Schaefer C, Biermann T, Hamann A, Schwarzloh B, Greten H, Ruther W, HansenAlgenstaedt N. Microvascular alterations in diabetic mice correlate with level of hyperglycemia. Diabetes. 2003;52:542-549.

124. Du XL, Edelstein D, Dimmeler S, Ju Q, Sui C, Brownlee M. Hyperglycemia inhibits endothelial nitric oxide synthase activity by posttranslational modification at the Akt site. J Clin Invest. 2001;108:1341-1348.

125. Nieuwdorp M, van Haeften TW, Gouverneur MC, Mooij HL, van Lieshout MH, Levi M, Meijers JC, Holleman F, Hoekstra JB, Vink H, Kastelein JJ, Stroes ES. Loss of endothelial glycocalyx during acute hyperglycemia coincides with endothelial dysfunction and coagulation activation in vivo. Diabetes. 2006;55:480-486. 
126. Nieuwdorp M, Mooij HL, Kroon J, Atasever B, Spaan JA, Ince C, Holleman F, Diamant M, Heine RJ, Hoekstra JB, Kastelein JJ, Stroes ES, Vink H. Endothelial glycocalyx damage coincides with microalbuminuria in type 1 diabetes. Diabetes. 2006;55:1127-1132.

127. Broekhuizen LN, Lemkes BA, Mooij HL, Meuwese MC, Verberne H, Holleman F, Schlingemann RO, Nieuwdorp M, Stroes ES, Vink H. Effect of sulodexide on endothelial glycocalyx and vascular permeability in patients with type 2 diabetes mellitus. Diabetologia. 2010;53:2646-2655.

128. Title LM, Cummings PM, Giddens K, Nassar BA. Oral glucose loading acutely attenuates endothelium-dependent vasodilation in healthy adults without diabetes: an effect prevented by vitamins $C$ and E. J Am Coll Cardiol. 2000;36:2185-2191.

129. Granger DN. Ischemia-reperfusion: mechanisms of microvascular dysfunction and the influence of risk factors for cardiovascular disease. Microcirculation. 1999;6:167-178.

130. Seal JB, Gewertz BL. Vascular dysfunction in ischemia-reperfusion injury. Ann Vasc Surg. 2005;19:572-584.

131. Oliver MG, Specian RD, Perry MA, Granger DN. Morphologic assessment of leukocyte-endothelial cell interactions in mesenteric venules subjected to ischemia and reperfusion. Inflammation. 1991;15:331-346.

132. Kurose I, Argenbright LW, Wolf R, Lianxi L, Granger DN. Ischemia/reperfusion-induced microvascular dysfunction: role of oxidants and lipid mediators. Am J Physiol. 1997;272:H2976-2982.

133. Vollmar B, Glasz J, Menger MD, Messmer K. Leukocytes contribute to hepatic ischemia/reperfusion injury via intercellular adhesion molecule-1-mediated venular adherence. Surgery. 1995;117:195200.

134. Beuk RJ, Heineman E, Tangelder GJ, Quaedackers JS, Marks WH, Lieberman JM, oude Egbrink MG. Total warm ischemia and reperfusion impairs flow in all rat gut layers but increases leukocytevessel wall interactions in the submucosa only. Ann Surg. 2000;231:96-104.

135. Kupinski AM, Shah DM, Bell DR. Transvascular albumin flux in rabbit hindlimb after tourniquet ischemia. Am J Physiol. 1993;264:H901-908.

136. Rubio-Gayosso I, Platts SH, Duling BR. Reactive oxygen species mediate modification of glycocalyx during ischemia-reperfusion injury. Am J Physiol Heart Circ Physiol. 2006;290:H2247-2256.

137. Chappell D, Dorfler N, Jacob M, Rehm M, Welsch U, Conzen P, Becker BF. Glycocalyx protection reduces leukocyte adhesion after ischemia/reperfusion. Shock. 2010;34:133-139.

138. Snoeijs MG, Vink H, Voesten N, Christiaans MH, Daemen JW, Peppelenbosch AG, Tordoir JH, PeutzKootstra CJ, Buurman WA, Schurink GW, van Heurn LW. Acute ischemic injury to the renal microvasculature in human kidney transplantation. Am J Physiol Renal Physiol. 2010;299:F1134-1140.

139. Beresewicz A, Czarnowska E, Maczewski M. Ischemic preconditioning and superoxide dismutase protect against endothelial dysfunction and endothelium glycocalyx disruption in the postischemic guinea-pig hearts. Mol Cell Biochem. 1998;186:87-97.

140. Ward BJ, Donnelly JL. Hypoxia induced disruption of the cardiac endothelial glycocalyx: implications for capillary permeability. Cardiovasc Res. 1993;27:384-389.

141. van den Berg B, Vink H. Glycocalyx perturbation: cause or consequence of damage to the vasculature? Am J Physiol Heart Circ Physiol. 2006;290:H2174-2175.

142. Hansson GK. Inflammation, atherosclerosis, and coronary artery disease. N Engl J Med. 2005;352:1685-1695.

143. Ross R. Atherosclerosis--an inflammatory disease. N Engl J Med. 1999;340:115-126.

144. Libby P. Inflammation in atherosclerosis. Nature. 2002;420:868-874.

145. Nagy N, Freudenberger T, Melchior-Becker A, Rock K, Ter Braak M, Jastrow H, Kinzig M, Lucke S, Suvorava T, Kojda G, Weber AA, Sorgel F, Levkau B, Ergun S, Fischer JW. Inhibition of hyaluronan synthesis accelerates murine atherosclerosis: novel insights into the role of hyaluronan synthesis. Circulation. 2010;122:2313-2322. 
146. Hjalmarsson C, Johansson BR, Haraldsson B. Electron microscopic evaluation of the endothelial surface layer of glomerular capillaries. Microvasc Res. 2004;67:9-17.

147. Rostgaard J, Qvortrup K. Electron microscopic demonstrations of filamentous molecular sieve plugs in capillary fenestrae. Microvasc Res. 1997;53:1-13.

148. Rostgaard J, Qvortrup K. Sieve plugs in fenestrae of glomerular capillaries--site of the filtration barrier? Cells Tissues Organs. 2002;170:132-138.

149. Barker AL, Konopatskaya O, Neal CR, Macpherson JV, Whatmore JL, Winlove CP, Unwin PR, Shore AC. Observation and characterisation of the glycocalyx of viable human endothelial cells using confocal laser scanning microscopy. Phys Chem Chem Phys. 2004;6:1006-1011.

150. van Zandvoort M, Engels W, Douma K, Beckers L, Oude Egbrink M, Daemen M, Slaaf DW. Twophoton microscopy for imaging of the (atherosclerotic) vascular wall: a proof of concept study. $J$ Vasc Res. 2004;41:54-63.

151. Robinson CJ, Stringer SE. The splice variants of vascular endothelial growth factor (VEGF) and their receptors. J Cell Sci. 2001;114:853-865.

152. Lopez-Casillas F, Wrana JL, Massague J. Betaglycan presents ligand to the TGF beta signaling receptor. Cell. 1993;73:1435-1444.

153. Yamashita K, Fukushima K. The carbohydrate recognition by cytokines modulates their physiological activities. Glycoconj J. 2004;21:31-34.

154. Spillmann D, Witt D, LindahI U. Defining the interleukin-8-binding domain of heparan sulfate. J Biol Chem. 1998;273:15487-15493.

155. Salek-Ardakani S, Arrand JR, Shaw D, Mackett M. Heparin and heparan sulfate bind interleukin-10 and modulate its activity. Blood. 2000;96:1879-1888.

156. Ali S, Fritchley SJ, Chaffey BT, Kirby JA. Contribution of the putative heparan sulfate-binding motif BBXB of RANTES to transendothelial migration. Glycobiology. 2002;12:535-543.

157. Hoogewerf AJ, Kuschert GS, Proudfoot AE, Borlat F, Clark-Lewis I, Power CA, Wells TN. Glycosaminoglycans mediate cell surface oligomerization of chemokines. Biochemistry. 1997;36:1357013578. 



\section{Endothelial glycocalyx structure}

\section{in the intact carotid artery}

A two-photon laser scanning microscopy study

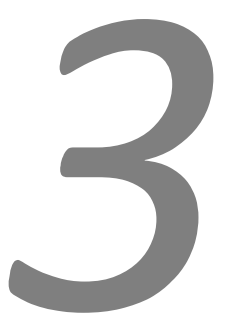

Published as

Sietze Reitsma, Mirjam G.A. oude Egbrink, Hans Vink, Bernard M. van den Berg, Valéria Lima Passos, Wim Engels, Dick W. Slaaf, Marc A.M.J. van Zandvoort 


\begin{abstract}
The endothelial glycocalyx (EG) is the carbohydrate-rich luminal lining of endothelial cells that mediates permeability and blood cell-vessel wall interactions. To establish an atheroprotective role of the EG, adequate imaging and quantification of its properties in intact, viable, atherogenesis-prone arteries is needed. Carotid arteries of C57BI6/J mice ( $n=22$ ) were isolated including the bifurcation, mounted in a perfusion chamber, and perfused with fluorescent lectin wheat germ agglutininfluorescein isothiocyanate. The EG was visualized through the vessel wall using two-photon laser scanning microscopy. An image quantification protocol was developed to assess EG thickness, which was sensitive to hyaluronidase-induced changes. In the lesion-protected common carotid artery, EG thickness was found to be $2.3 \pm 0.1 \mu \mathrm{m}$ (mean \pm SEM), while the surface area devoid of (wheat germ agglutinin-sensitive) EG was $8.9 \pm 4.2 \%$. Data from the external carotid artery were simi$\operatorname{lar}(2.5 \pm 0.1 \mu \mathrm{m} ; 9.1 \pm 5.0 \%)$. In the atherogenesis-prone internal carotid artery the EG-devoid surface area was significantly higher $(27.4 \pm 5.5 \%, p<0.05)$; thickness at the remaining areas was $2.5 \pm 0.1 \mu \mathrm{m}$. The EG can be adequately imaged and quantified using two-photon laser scanning microscopy in intact, viable mounted carotid arteries. Spatial EG differences could underlie atherogenesis.
\end{abstract}




\section{Introduction}

The endothelial glycocalyx (EG) is an intricate mesh of proteoglycans and glycoproteins located on the luminal side of vascular endothelial cells. ${ }^{1}$ Extending into the lumen, it forms the interface between flowing blood and the vessel wall. Its importance as such has been increasingly appreciated over the past decades. Recent studies imply the EG to be involved in control of permeability and blood cell-vessel wall interactions. $^{2-5}$ Therefore, damage to the EG is proposed to increase the risk for development of atherosclerotic lesions. ${ }^{1,6}$

To address a possible (athero-)protective role of the EG, adequate in vivo visualization and quantification of its properties are needed. This can be achieved by indirect imaging, where the EG is defined as an exclusion zone for red blood cells or certain plasma column markers, such as large dextrans. ${ }^{7}$ However, this method is most suited for smaller vessels, which do not develop atherosclerotic lesions. Imaging the EG in larger arteries of small rodents can be realized using electron microscopy. ${ }^{2,-11}$ However, this requires processing and slicing of the vessels, resulting in dehydration of the EG and loss of three-dimensional context. Another approach to visualize the EG in larger arteries is confocal laser scanning microscopy (CLSM), which has been applied to isolated carotid arteries, ${ }^{12}$ providing valuable data on EG composition. Since the penetration depth of CLSM does not suffice to image through the vessel wall, arteries need to be cut open longitudinally to allow imaging, which might compromise EG structure and cannot be applied in vivo. Therefore, we set out to develop a method to visualize the EG in intact, viable large arteries using two-photon laser scanning microscopy (TPLSM).

TPLSM depends on the excitation of a fluorophore by simultaneous arrival of two photons, instead of one in conventional (and confocal) fluorescence microscopy. The wavelength of the excitation light (about $800 \mathrm{~nm}$ ) is roughly double that of the light used in conventional fluorescence microscopy. This reduces scattering of excitation light and, as a result, increases penetration depth. Furthermore, two-photon excitation only occurs in a small volume in the focal plane; all emitted fluorescent signal originates from this focal point. Hence, scattering of emission light has no influence on the definition of the signal, and, therefore, no pinholes are needed. Taken together, TPLSM provides good penetration depth, optical sectioning and subcellular resolution, enabling imaging in intact, pressurized, viable arteries of rodents. $^{13}$ 
The resolution of TPLSM is, however, limited. Theoretically it is about $0.4 \times 0.4 \times$ $0.9 \mu \mathrm{m}$ in $\mathrm{x}, \mathrm{y}$, and $\mathrm{z}$ direction, respectively, given the objective and excitation wavelength used; also, the tissue under study is of influence. By applying subresolution-scaled fluorescent beads intraluminally, we have found that the xyz resolution of our system at the level of the endothelium is about $0.5 \times 0.5 \times 2.1 \mu \mathrm{m}$ when imaging through the vessel wall of carotid arteries. ${ }^{14}$ This approximates the thickness of the EG itself. ${ }^{12,13}$ Furthermore, experimental settings and resulting images are often not comparable between measurements as they depend on conditions affecting the yield of fluorescence and on vessel properties. These considerations need to be taken into account when quantifying three-dimensional image data.

Imaging of the EG can be achieved by using fluorescently labeled lectins. These (mostly) plant-derived proteins bind to specific sugar moieties. A wide variety of lectins is commercially available and can be used to investigate (endothelial) glycocalyx composition in different models. ${ }^{15-18}$ When applying lectins to the EG, one should take into account both the specificity of the lectin and the abundance of its target in the EG. Furthermore, these sugar-binding proteins need to be able to penetrate (permeate) the EG in order to provide labeling. Previous pilot TPLSM experiments indicated that in mouse carotid arteries the EG can be labeled and visualized using the lectin wheat germ agglutinin (WGA). ${ }^{13}$

In our search for a method to quantify EG properties in intact large arteries, the objectives of the present study were multifold. (1) To further explore the binding characteristics of WGA and its applicability as a general EG label. (2) To test whether EG thickness can be adequately quantified at the current axial resolution with image-derived thresholds when imaged through the intact vessel wall. (3) To test whether WGA-based EG staining and quantification is sensitive to structural changes, as induced by a glycocalyx-degrading enzyme. (4) To image the EG in the common, external and internal carotid artery. These locations are exposed to different shear stress patterns in vivo, resulting in different susceptibility for atherosclerosis, ${ }^{19}$ and differences in glycocalyx structure and function. ${ }^{12}$ 


\section{Materials and Methods}

\section{Data acquisition}

\section{Isolation and mounting of carotid arteries}

All experiments conducted were in line with institutional guidelines and approved by the local ethics committee on the use of laboratory animals. Male 8- to 25week-old C57BI6/J mice $(n=22)$ were euthanized using $\mathrm{CO}_{2}$. Their carotid arteries were processed as described elsewhere. ${ }^{13}$ Isolated carotid arteries including the bifurcation were mounted on two glass micropipettes in a custom-built perfusion chamber (IDEE, Maastricht, the Netherlands) filled with a modified Hanks' balanced salt solution ( $\mathrm{pH} 7.4$ ), containing in $\mathrm{mM}$ : $\mathrm{NaCl} 137, \mathrm{HEPES} 15$, glucose $5.5, \mathrm{KCl} 5.3$, $\mathrm{CaCl}_{2} 2.5, \mathrm{Na}_{2} \mathrm{HPO}_{4} 0.34, \mathrm{KH}_{2} \mathrm{PO}_{4} \mathrm{0.44}$, and $\mathrm{MgSO}_{4}$ 1.1. The internal or external carotid artery and smaller side branches were ligated, whilst the remaining large side branch of the carotid bifurcation and the common carotid artery were mounted on the micropipettes. Vessel processing, labeling, and imaging were performed at room temperature, which ensures vascular cell viability for at least $4 \mathrm{~h}^{13}$ After labeling procedures, a transmural pressure of $50-80 \mathrm{mmHg}$ was applied. At these pressures, vessel diameters are comparable to the in vivo situation, as we have deduced from the recorded image stacks using a diameter estimation algorithm. ${ }^{20}$

\section{Lectin labeling}

Mounted carotids were labeled luminally with $2.6 \mu \mathrm{M}$ WGA linked to fluorescein isothiocyanate (WGA-FITC; Sigma Aldrich, Zwijndrecht, the Netherlands). WGA-FITC labels glucosamine-based glycosaminoglycans in the EG, such as heparan sulfate and hyaluronan, and also has affinity for sialic acids. SYTO41 (5 $\mu \mathrm{M}$; Molecular Probes, Leiden, the Netherlands) was used as a counter stain for vascular cell nuclei. Probes were diluted in phosphate-buffered saline (PBS) and perfused at a rate of $1 \mathrm{ml} / \mathrm{h}$ for $30 \mathrm{~min}$ (low shear; approximately 0.02 Pa when assuming a diameter of $500 \mu \mathrm{m})$.

\section{Hyaluronidase challenge}

Five vessels were challenged with the enzyme hyaluronidase (type IV-S, Sigma Aldrich). After heating the perfusion chamber to $37{ }^{\circ} \mathrm{C}$, hyaluronidase $(100 \mathrm{U} / \mathrm{ml}$ in PBS) was perfused at a rate of $1 \mathrm{ml} / \mathrm{h}$ for either $30(\mathrm{n}=3)$ or $60 \mathrm{~min}(\mathrm{n}=2)$. Because of the dead volume in the perfusion chamber, effective hyaluronidase challenge times were approximately 15 and $45 \mathrm{~min}$. Dead volume is approximately $0.25 \mathrm{ml}$ and consists of fluid in the tubing proximal to the mounted vessel. Negative con- 
trols were performed using PBS perfusion only under the same conditions. Although literature suggests a more profound removal of WGA-FITC-stained structures using neuramindase, ${ }^{21}$ the choice for hyaluronidase was made based on its specificity for glucosamine-based glycosaminoglycans, towards which our attention is aimed.

\section{Two-photon laser scanning microscopy}

Imaging was performed on a Nikon E600FN microscope (Nikon, Tokyo, Japan), coupled to a standard Bio-Rad 2100 MP two-photon system (Bio-Rad, Hemel Hempstead, UK) with a $120 \pm 20$-fs-pulsed Ti:Sapphire laser as excitation source at 800 nm (Spectra Physics Tsunami, Mountain View, Calif., USA). A 60x water dipping objective (numerical aperture 1.0) was used. The field of view (FOV) was approximately $200 \times 200 \mu \mathrm{m}^{2}$, captured into $512 \times 512$ pixels at a frame rate of $1 \mathrm{~Hz}$. These settings (suboptimal with respect to the Nyquist criterion) are a result of balancing sampling rate with experimentation time and phototoxicity. ${ }^{13}$ Fluorescent signals from SYTO41 and FITC were detected by two photo multiplier tubes (PMTs), tuned to match the emission spectrum of the probes and to minimize spectral leakthrough: PMT1 (SYTO41) 420 - 470 nm; PMT2 (FITC) 510 - 550 nm. Single images were recorded in the $x y$ plane; series of $x y$-images were collected at successive depths (z stack; Figure 3.1).Step size (distance in z direction between xy planes) was either 0.45 or $0.60 \mu \mathrm{m}$. Photon counting was applied, which uses the factorydetermined noise levels of the PMTs to improve the signal-to-noise ratio (SNR).

\section{Image data quantification protocol}

TPLSM data were analyzed with Imagepro software package (version 6.1; Media Cybernetics, Silver Spring, Md., USA) including the three-dimensional reconstructor set (version 5.1). For image computations, tailored software was developed within a Matlab environment (version 7.01; The MathWorks Inc., Natick, Mass., USA). No z stack deconvolution or compression was applied, as this requires accurate definition of the point spread function (PSF). This is not feasible, as it varies over the vessel wall with changing local refractive indices.

\section{Noise}

For each PMT, a region of interest was selected in the lumen of the vessel (no WGA-FITC or SYTO41 labeling). Noise values were based on the pixel intensity histogram from this region of interest (noise histogram) and defined as the intensity value which covered $98 \%$ of the noise histogram. The background noise values in 


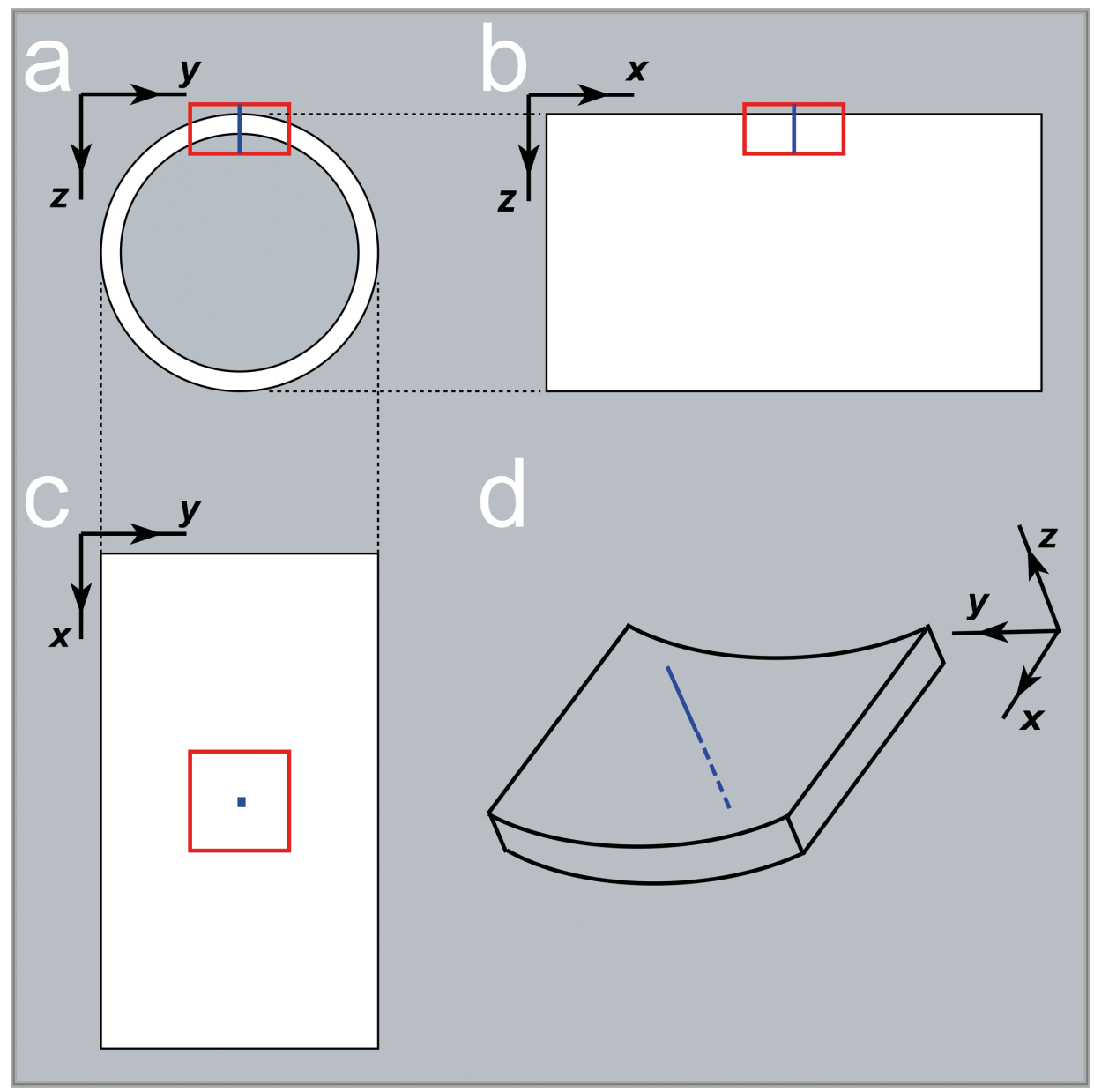

Figure 3.1 Scanning directions.

Schematic drawing representing a blood vessel drawn in three planes: $a$ transversal view, yz; $b$ side view, xz; $c$ top view, xy. The red box in all planes represents the scanned volume, as shown in other figures. Recordings were made with the $x$ axis parallel to flow (longitudinal direction) and the $y$ axis perpendicular to flow (transversal direction); the $z$ axis represents scanning depth. xy sections were obtained at successive depth (z stack).

$d$ From a z stack, a three-dimensional reconstruction can be made, which is presented inversely to show the endothelial side of the vessel segment.

From this figure it is clear that a line in z direction corresponds with a single position in the xy plane (blue line/dot).

these 8-bit images (maximum intensity value 255 ) were in the range of $6-12$. All information equal to or lower than background was ignored during further image analysis. 


\section{Position of the endothelial plane}

The position of the endothelial surface was calculated from the SYTO41 data (cell nuclei) in PMT1. The maximum of pixel intensities in PMT1 was determined per line in $z$ direction over a depth of $6 \mu \mathrm{m}$, starting from the first luminal pixel with an intensity value above threshold. The position of the endothelial surface was defined as the first pixel whose intensity was higher than half the line maximum. In empty lines (with no signal above threshold) the position of the endothelium was assumed to be equal to the neighboring lines in longitudinal direction, as vessel segment curvature is more pronounced in transversal direction (Figure 3.1). The obtained endothelial surface was smoothed using a 3-by-3 neighborhood median filter.

\section{EG thickness calculation}

Thickness of the EG was determined per line in z direction from the WGA-FITC data in PMT2. The maximum intensity value of the pixels located luminally from the endothelial surface was calculated. The half of the maximum intensity value (HM) served as threshold for EG detection. If the HM was equal to or lower than the assessed background noise, the line was considered empty. However, if the HM was above background, all luminal pixels with an intensity value equal to or higher than the HM were counted up to give the glycocalyx thickness for that line. The step size of the $z$ stack defined pixel dimension in $z$ direction and, hence, the lowest thickness that could be detected (either 0.45 or $0.60 \mu \mathrm{m}$ ).

Analysis of the complete vessel segment recording resulted in a two-dimensional matrix with $\mathrm{x}$ and $\mathrm{y}$ dimensions matching those from the recorded $\mathrm{z}$ stack. Each position in this matrix indicated the thickness of the EG for the corresponding line in the $z$ stack (Figure 3.1, blue line). The median glycocalyx thickness was derived from the thickness distribution of this matrix. It is mathematically defined as the point where the cumulated thickness histogram reaches $50 \%$. This value was estimated using a splined interpolation curve plotted through the cumulated thickness histogram (Figure 3.4b). The percentage of endothelial surface area that was lacking sufficient WGA-FITC signal to be picked up by the analysis protocol (surface area lacking signal) was defined as all empty lines divided by the total number of lines $x$ $100 \%$. Per position, the maximum intensity of the EG signal was also recorded, from which an overall SNR could be calculated. SNR was defined as the average HM divided by the background noise value. Images with a SNR below 2.5 overestimated EG thickness and were not used for further analysis. 


\section{Statistical analysis}

Recordings were made in both carotid arteries (common, internal, and external) of each mouse. If available, data from both arteries per mouse were averaged per location. Still, the data were not fully balanced, i.e., measurements could not be obtained for all vessel locations of all mice. Some recordings were discarded due to a SNR $<2.5$. In other cases, smaller side branches from the carotid bifurcation could not be identified and ligated successfully, thus impairing the application of a transmural pressure of $50-80 \mathrm{mmHg}$. To bypass the inconvenience of missing data as well as to account for the dependence of the measurements, a random intercept model analysis was conducted, ${ }^{22}$ in order to evaluate the effect of vessel location on EG thickness and surface area lacking signal. Mice and vessel location were taken as random and fixed effect factors, respectively. With this approach all available data were used for parameter estimation and not only of those mice with complete data. Model parameters were estimated via the restricted maximum likelihood and the significance level was set at 5\%. Statistical analyses were performed using the SPSS software package (version 15.0; SPSS Inc., Chicago, III.,USA).

\section{Results}

\section{WGA labeling characteristics}

Carefully dissected and mounted carotid arteries perfused with WGA-FITC showed very fine labeling along the membrane lining of the endothelial cells, with some labeling over the endothelial cells. Occasionally, brightly labeled spots could be detected (Figure 3.2a). Assuming that the EG is normally not permeable to WGA (43 kDa), the observed cell membrane pattern could be an effect of convective flow, leading to a local rise of WGA concentration to detectable levels. In other words, the pattern could reflect nonspecific labeling. To achieve complete labeling of the EG, its permeability was increased by flushing a small air bubble through the mounted vessel (standardized maneuver: volume $0.05-0.1 \mathrm{ml}$, exposure time 3 $5 \mathrm{~s})$. Indeed, WGA labeling changed acutely from the cell membrane pattern into a bright layer covering the endothelium (Figures 3.2b-d). In some carotid arteries, no deliberate increase of EG permeability was needed in order to achieve complete EG labeling by WGA. 


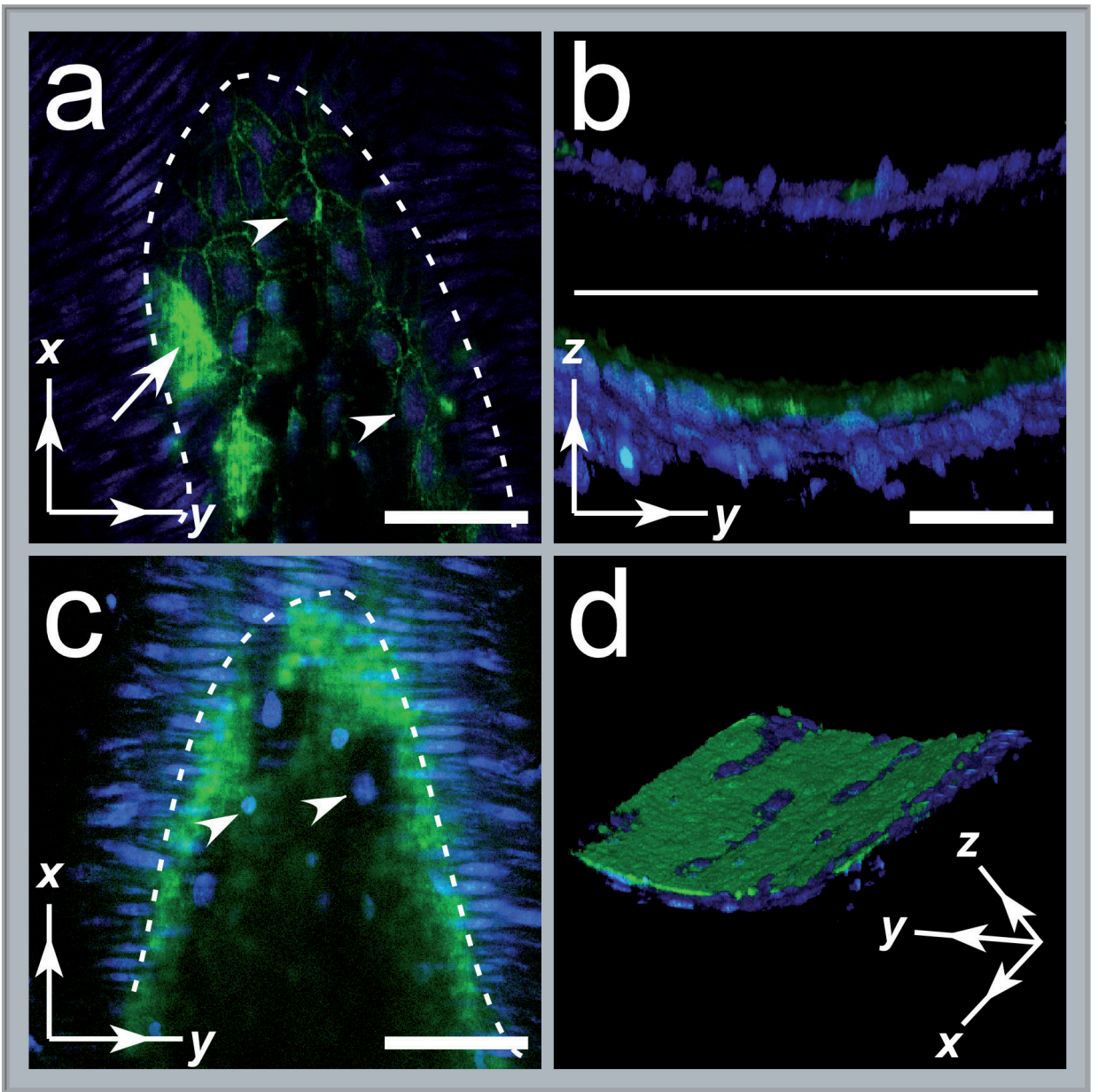

Figure 3.2 WGA labeling characteristics.

Optical sections from mounted isolated common carotid arteries, labeled intraluminally with WGA-FITC (2.6 $\mu \mathrm{M}$, green) and $5 \mu \mathrm{M}$ SYTO41 (blue, cell nuclei), before and after increase of glycocalyx permeability.

$a$ xy section showing an endothelial cell membrane pattern that can be identified at low intensity. To visualize this, gain is roughly doubled, leading to pixel saturation in some brightly labeled spots (arrow).

$b$ yz sections before (upper) and after (lower) exposure to a small air bubble. An acute increase of WGAFITC labeling is observed at the luminal side of the endothelium, indicating that the glycocalyx has become accessible to WGA.

$c$ xy section after exposure to air. Bright green labeling is now visible.

$d$ Three-dimensional reconstruction from (c) showing that the brightly labeled structure is a layer covering the endothelium. The surface area lacking signal in this recording is $15 \%$.

Dotted lines indicate the transition from endothelium to media (smooth muscle cells). Arrowheads indicate endothelial cell nuclei. Scale bars $=50 \mu \mathrm{m}$. Scanned volume $(d)$ is $200 \times 200 \times 40 \mu \mathrm{m}$. 


\section{Determination of thickness with limited axial resolution}

Our next goal was to quantify the glycocalyx layer imaged through the vessel wall. However, the axial resolution of our system at the depth of the EG is $2.1 \mu \mathrm{m}$, calculated as the full width at HM (FWHM) of the point spread function (PSF) in z direction. ${ }^{14}$ This rather poor resolution dictates elongation of objects in $z$ direction. Deconvolution algorithms may be employed to overcome this effect. However, deconvolution requires accurate determination of the PSF over the vessel wall, which is not feasible. Therefore, we simulated a 3- $\mu$ m-thick layer of 101 points, located $0.03 \mu \mathrm{m}$ from each other, and added a FWHM-PSF of $2.1 \mu \mathrm{m}$ to each point. This way we could determine whether this resolution is sufficient for quantification of EG thickness, despite object elongation. When assuming a homogeneous intensity distribution, the intensity functions for each point can be added up to give the theoretical intensity distribution of the simulated layer (Figure 3.3a). When the HM intensity value is used as cut-off (FWHM), the thickness of the layer could be determined accurately. Furthermore, the simulated intensity profile is comparable to the actual data (Figures 3.3a and b). Thus, the principle of FWHM-based signal quantification allows accurate measurements of objects in z direction, despite limited axial resolution. It was therefore used as basis for an image quantification protocol.

\section{Image quantification}

Image data analysis showed leak-through from the SYTO41 signal (mainly PMT1) into the glycocalyx data in PMT2 (Figure 3.3b). Moreover, WGA-FITC could occasionally be found in the adventitia, when the probe had leaked through incompletely ligated smaller side branches. In order to extract the EG signal from the data in PMT2, information in PMT1 (cell nuclei) was used to pinpoint the location of the endothelium per line in $z$ direction. However, simply determining the maximum intensity of the $z$ line is not valid, as SYTO41 signal is distributed heterogeneously over the vessel wall (Figure 3.3c). Taking the first pixel above the noise threshold from the direction of the lumen is not valid either, as objects are elongated in $z$ direction (as described by the PSF; Figure 3.3a) and the position of the endothelium determined this way would be too far into the lumen. Thus, to circumvent these limitations, the local maximum from the endothelial cell nuclei was used (Materials and Methods; Figure 3.3c, black arrow), which was not influenced by adventitial SYTO41 signal (Figure 3.3c, grey arrow). In lines without an endothelial cell nucleus, the information of the smooth muscle cell nuclei was used. Thus, the position of the endothelial surface could be determined despite a heterogeneous SYTO41 signal distribution (Figure 3.4a). 

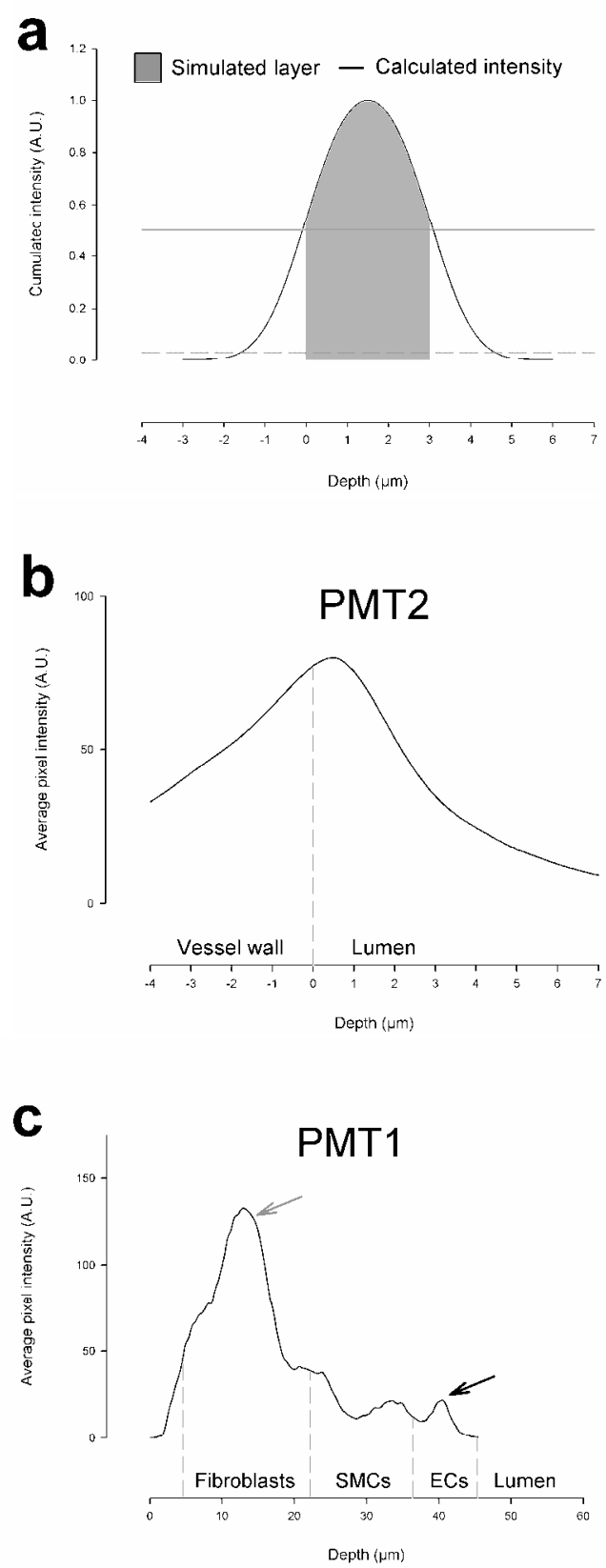

Figure 3.3 Signal analysis at limited z resolution.

a Cumulated intensity profile of a simulated 3- $\mu \mathrm{m}$-thick layer consisting of 101 points each with a point spread function of $2.1 \mu \mathrm{m}$ in $\mathrm{z}$ direction. Using the background noise as a threshold to calculate the thickness of the layer would result in huge overestimation (dotted line), while using the FWHM approximates true layer thickness (solid line). Based on this finding, the latter is used as basis for the image quantification protocol.

$b$ Line intensity profile of PMT2 (WGA-FITC, and SYTO41 leak-through) over the endothelium. The luminal PMT2 signal resembles the simulated profile from $a$, with its maximum close to the location of the endothelium (dotted line). The abluminal signal shows evidence of SYTO41 leakthrough.

c Line intensity profile of PMT1 (SYTO41, vascular cell nuclei) over the vessel wall. SYTO41 fluorescence is heterogeneously distributed, with highest intensities in the adventitia (fibroblasts, grey arrow). A local maximum is needed (black arrow) to locate the endothelial cells. SMCs: smooth muscle cells; $E C s$ : endothelial cells. 


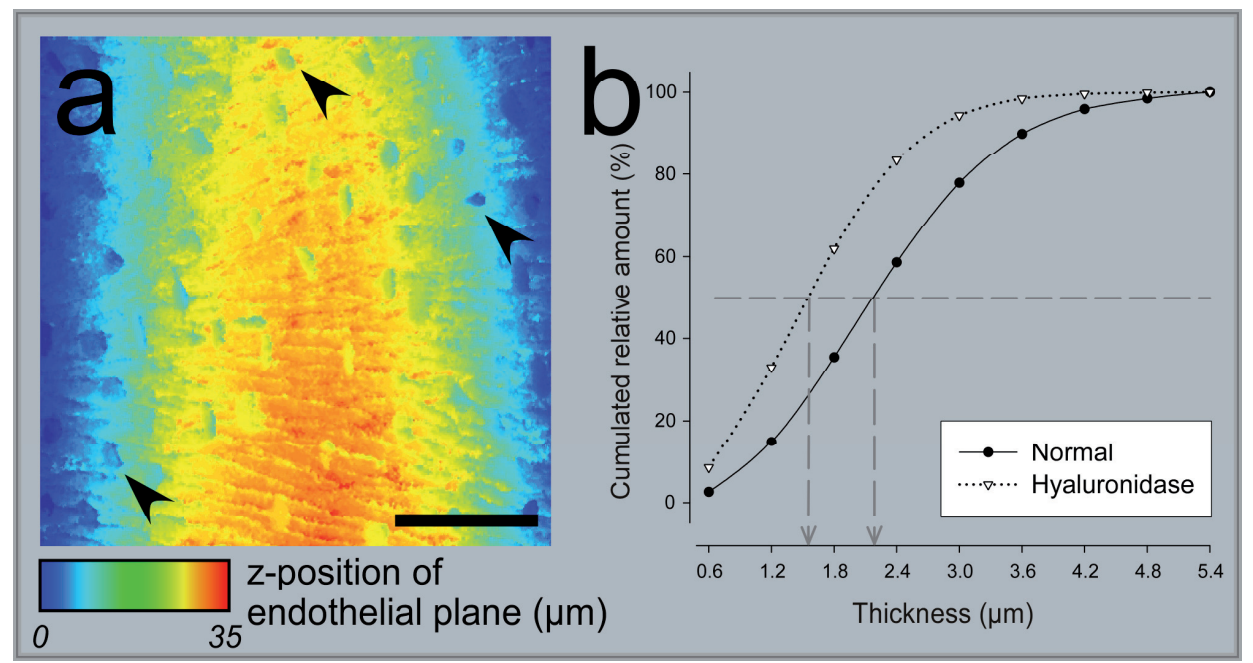

Figure 3.4 Image analysis protocol.

a An xy projection of the location of the endothelial surface, calculated from the data in PMT1 (SYTO41 fluorescence). Colors indicate relative depth (z position). Endothelial cells can be recognized as structures extending from the general surface (arrowheads). Note the slight curvature in the vessel. Bar represents $50 \mu \mathrm{m}$.

$b$ Distribution of glycocalyx thickness in control conditions (solid) and after 45 min of hyaluronidase treatment $(100 \mathrm{U} / \mathrm{ml}$; dotted). Median glycocalyx thickness is defined as the thickness where the cumulated number is $50 \%$ (arrows). Hyaluronidase treatment shifted the entire thickness distribution to the left and thus reduced median thickness (from 2.2 to $1.6 \mu \mathrm{m}$ ).

All pixels located abluminally from the endothelial surface were regarded intima, media or adventitia. The PMT2 data (green; either WGA-FITC or SYTO41 leakthrough) from these pixels were ignored during further analysis. Through this procedure, an accurate EG signal was retrieved from the data in PMT2. This could then be quantified per line in $z$ direction to give the thickness distribution of the EG over the scanned vessel segment. This distribution was found to be skewed to the right (i.e., there are relatively more areas with thinner glycocalyx present). Because of this skewed distribution, the median thickness (and not the average) is a more appropriate representative of the central location of the EG thickness distribution. By fitting a splined curve through the cumulative plot, the median value was defined as the thickness where the cumulative number is $50 \%$ (Figure $3.4 \mathrm{~b}$ ). Furthermore, the surface area lacking signal was calculated. 
Table 3.1 Estimated mean values from random intercept model

\begin{tabular}{lllll}
\hline \hline $\begin{array}{l}\text { Location in } \\
\text { carotid artery }\end{array}$ & $\mathrm{n}$ & $\begin{array}{l}\text { Thickness } \\
(\mu \mathrm{m})\end{array}$ & $\begin{array}{l}\text { Surface area lacking } \\
\text { signal }(\%)\end{array}$ & $\begin{array}{l}\text { Surface area devoid of WGA- } \\
\text { sensitive EG }(\%)\end{array}$ \\
\hline \hline Common & 16 & $2.3 \pm 0.1$ & $20.1 \pm 3.9$ & $8.9 \pm 4.2$ \\
Internal & 9 & $2.5 \pm 0.1$ & $43.5 \pm 5.2^{*}$ & $27.4 \pm 5.5^{* *}$ \\
External & 10 & $2.5 \pm 0.1$ & $17.6 \pm 4.7$ & $9.1 \pm 5.0$ \\
\hline
\end{tabular}

Data are presented as mean \pm SEM. Pair-wise comparisons were made using Bonferroni correction. Numbers differ between vessel locations due to technical issues, see Materials and Methods.* $p<0.01$ vs. common and external; ${ }^{* *} p<0.05$ vs. common and external.

Table 3.1 lists the data on EG thickness and surface area lacking signal per vessel location. Mean EG thickness in the common carotid artery was found to be $2.3 \pm$ $0.1 \mu \mathrm{m}$, which did not differ significantly from the internal and external carotid artery. However, the amount of surface area lacking signal was significantly higher in the internal carotid artery (43.5\%) as compared to the common and external carotid artery $(20.1$ and $17.6 \%$, respectively; $p<0.01)$.

To test the sensitivity of the developed image quantification protocol, hyaluronidase was used to degrade the EG. In the common carotid artery, a hyaluronidase challenge of 15 min did not alter WGA-labeled EG thickness, while the surface area lacking signal increased threefold on average $(n=3)$. After 45 min of enzyme treatment, thickness was decreased by $25 \%$ (Figure $3.4 \mathrm{~b}$ ), while the surface area lacking signal increased threefold $(n=2)$. No changes in EG composition were found in negative controls (data not shown).More detailed studies of the surface areas lacking signal were performed. In some of these areas, increasing the gain during offline analysis revealed the previously observed cell membrane pattern, its intensity too low to be picked up in the described analysis protocol. In these cases, lack of signal was based on (retained) impermeability to WGA. On the other hand, some areas lacking signal showed no evidence of cell membrane pattern staining after increasing the gain. These areas were regarded as devoid of (WGA-sensitive) EG. After correction of the surface area lacking signal for this distinction (impermeable vs. devoid of EG), the internal carotid artery was found to have a significantly higher amount of EG-devoid surface area as compared to both the common and the external carotid artery (Table 3.1). No significant differences were found in the amount of cell membrane pattern staining. Furthermore, the changes in surface area lacking signal as induced by hyaluronidase were found to be completely due to an increase in EG-devoid area. 


\section{Discussion}

In isolated, intact, viable and pressurized mouse carotid arteries the EG was visualized using TPLSM. Perfusing vessels with the fluorescent lectin WGA-FITC resulted in a distinctive cell membrane pattern at low intensity (Figure 3.2a).

After perfusion of the vessel with an air bubble, WGA-FITC perfusion resulted in a complete labeling of the EG layer (Figures 3.2b - d), which covered approximately $80 \%$ of the endothelial surface in common carotid arteries. This indicates that WGA-FITC is indeed suitable as a general label for the EG. However, its applicability in vivo is limited, as WGA does not label the EG in control conditions. Similar findings have been described previously in a study by Baldwin and Winlove ${ }^{23}$ comparing binding characteristics of WGA in rabbit aortas flushed with saline or with serum. These vessels were prepared for electron microscopy, resulting in a dehydrated and collapsed EG. ${ }^{1}$ No WGA permeation was found in the presence of serum. Perfusion with saline enabled permeation of WGA-gold particles to the EG. This finding is comparable to our data as shown in Figure 3.2a, where perfusion with WGA-containing PBS gives rise to minimal labeling at the level of the EG. Moreover, Baldwin and Winlove ${ }^{23}$ describe an approximately threefold increase of WGA density in the cannulated regions of the aorta. There, endothelial cells appeared damaged, but still had an intact plasmalemma. This is comparable to the greatly increased labeling of WGA-FITC after air bubble perfusion (Figures $3.2 b-d$ ). We conclude that the EG in freshly excised and mounted arteries is not labeled by WGA-FITC despite its low molecular weight. Other probes of similar weight have been shown to be excluded from the endothelium as well in our setup. ${ }^{24}$ It is not known whether air bubble perfusion alters the depth, as well as the labeling, of the EG.

Through tailored programming, WGA-FITC-labeled EG dimensions could be quantified adequately despite limited resolution in z direction when imaged through the vessel wall. Furthermore, the analysis protocol allows investigator-independent three-dimensional analysis of EG properties. Instead of using overall set thresholds, ${ }^{13}$ it allows the use of image-derived thresholds, and, thus, it no longer ignores areas with lower pixel intensities. Moreover, the influence of signal leak-through from SYTO41 into PMT2 is greatly reduced in the new analysis. Compared to the previously reported pilot data on WGA-based EG staining, ${ }^{13}$ this is a major improvement.

The determined endothelial surface ensures that only luminal WGA-FITC signal is used for EG measurements. In parts of the scanned volume with no endothelial cell 
nucleus overlying the tunica media, the endothelial surface boundary is determined by signal from smooth muscle cell nuclei (also labeled with SYTO41). This way, WGA-FITC labeling inside endothelial cells could not be separated from external labeling. However, this will hardly influence thickness measurements, as the dimensions of the endothelial cell plasma in z direction are very limited compared to EG properties. ${ }^{2}$ Also, the EG as a whole is imaged, and no differentiation between membrane-bound and soluble components of the EG can be made.

Some limitations apply to the analysis protocol. (1) The scanned vessel segments are regarded as being flat, while in fact a slight curvature is present, mostly in the yz plane (Figures 3.1 and 3.4a). Ideally, analysis of EG thickness would be performed in radial direction, instead of $z$ direction. However, this requires extensive programming. The angle between the radial direction and $z$ direction is largest at the edges of the FOV. Hence, the largest deviation of EG thickness can be expected there. However, no clear difference in thickness between the borders and the center of the FOV was found in our data. This suggests that the deviation induced by the analysis protocol at the current settings (diameter $>400 \mu \mathrm{m}$ and FOV $(200 \times 200$ $\times 40-60 \mu \mathrm{m}$ ) is smaller than the deviation of EG thickness itself. However, when imaging smaller vessels or using a larger FOV, the assumption that the vessel is flat may be violated. Also, data retrieved from vessel areas with large geometric variations (such as bifurcations) should be interpreted with care. (2) The vessels are assumed to be aligned horizontally; in other words, the $z$ axis should be perpendicular to the endothelial surface. Violation of this assumption would result in an overestimation of the thickness in $z$ direction. But, as angles between the $z$ axis and the longitudinal and transversal directions of the endothelial plane could be measured, we determined that the overestimation of EG thickness was always $<1 \%$. (3) When using FHWM-based calculations, pixel saturation should be avoided, as the true maximum and HM values will be obscured. In the presented data, the number of lines with more than one pixel with an intensity value of 255 was always $<3 \%$.

The described quantification protocol is sensitive to changes in EG thickness and endothelial coverage, as induced by a hyaluronidase challenge (Figure 3.4b). The measured effects are very similar to those reported in literature in various models. $^{2,4,21}$ Furthermore, EG properties were compared between vascular sites exposed to different shear stress patterns in vivo. No differences were found between the atherogenesis-protected common carotid and the external carotid artery. In the atherogenesis-prone internal carotid artery, EG thickness was comparable, but the EG-devoid surface area was significantly higher. In a previous study, based on CLSM in longitudinally cut open, unpressurized arteries, EG thickness was 
found to be $4.3 \mu \mathrm{m}$ in the common carotid artery and $2.3 \mu \mathrm{m}$ in the carotid artery sinus (i.e., part of the internal carotid artery). ${ }^{12}$ Our data show two major differences, which are discussed below.

(1) EG thickness in the common carotid artery is almost half of that obtained using CLSM. In the CLSM study, the demarcation of the endothelial cells was determined at peak fluorescence intensity. We have used FWHM instead, based on our model as depicted in Figure 3.3a. This will result in relatively lower EG thickness values, as the FHWM-determined endothelial surface is located more luminally than the one determined at peak intensity (Figure 3.3c). We estimate that this difference is about 1 or 2 z steps (i.e., up to $1.2 \mu \mathrm{m}$ ). Furthermore, it is not unlikely that the application of pressure in our model has led to compression of the EG and, thus, in a reduced thickness.

(2) No difference in EG thickness was found between the common and internal carotid artery in our study. The CLSM data were obtained using a smaller FOV $(20 \times$ 20 vs. $200 \times 200 \mu \mathrm{m}$ in our setup). Therefore, CLSM recordings could be made well within the carotid sinus. This location is known to be especially vulnerable to atherosclerosis. Although the larger FOV of the TPLSM setup has the advantage to scan and analyze large endothelial surfaces at once, such small anatomical locations cannot be identified separately in the current recordings. In future experiments, the FOV of the TPLSM system could be adjusted if a more detailed EG analysis is required. Interestingly, both CLSM and TPLSM studies showed a lower amount of labeling of the EG in the internal carotid artery as compared to the common.

Despite the differences between the results of the two methods, the TPLSM-based approach holds more potential for future studies as it enables imaging of EG properties in intact, viable arteries. ${ }^{13}$ There is no need to cut open the vessel of interest, and a physiological transmural pressure (and temperature) can be applied. The finding that TPLSM allows adequate imaging and quantification of small structures through the vessel wall is also promising for in vivo studies. The current use of WGA-FITC, however, seems less feasible for in vivo imaging of the EG. To this end, other lectins should be explored.

Our data indicate that EG permeability needs to be increased to enable specific WGA labeling. This raises the question whether really no EG is present at parts of the endothelial surface area lacking sufficient signal to be picked up by the analysis protocol. As some of these areas were found to be covered with the cell membrane pattern at low intensity, absence of labeling can in part be explained by a locally retained permeability barrier for WGA, even after perfusion with air. On the other hand, lack of WGA-FITC signal can also be caused by a loss of (WGA-sensitive) EG, 
such as was found after hyaluronidase treatment. Whether the (spontaneously) higher amount of EG-devoid surface area in the internal carotid artery reflects real absence of EG, a difference in EG composition, or a combination of both, remains to be elucidated.

\section{Conclusion}

In isolated carotid arteries of mice the EG can be labeled and imaged using fluorescently labeled WGA and TPLSM. Adequate quantification of EG thickness is possible despite a limited resolution in z direction. The described method allows quantification of changes in EG properties as induced by hyaluronidase treatment. The EG in the atherogenesis-prone internal carotid was determined to be as thick as in the common carotid, while the surface area that was devoid of (WGA-sensitive) EG was found to be significantly higher. As the latter indicates either local absence of EG or a difference in its composition, our data further solidify the hypothesis that differences in the EG could underlie the development of atherogenic plaques.

\section{Acknowledgements}

This research is supported by an NWO grant No. 902-16-276 and SenterNovem (BSIK 03033). 


\section{References}

1. Reitsma S, Slaaf DW, Vink H, van Zandvoort MA, Oude Egbrink MG. The endothelial glycocalyx: composition, functions, and visualization. Pflugers Arch. 2007;454:345-359.

2. van den Berg BM, Vink $H$, Spaan JA. The endothelial glycocalyx protects against myocardial edema. Circ Res. 2003;92:592-594.

3. Constantinescu AA, Vink H, Spaan JA. Endothelial cell glycocalyx modulates immobilization of leukocytes at the endothelial surface. Arterioscler Thromb Vasc Biol. 2003;23:1541-1547.

4. Henry $C B$, Duling BR. Permeation of the luminal capillary glycocalyx is determined by hyaluronan. Am J Physiol. 1999;277:H508-514.

5. Vink H, Constantinescu AA, Spaan JA. Oxidized lipoproteins degrade the endothelial surface layer: implications for platelet-endothelial cell adhesion. Circulation. 2000;101:1500-1502.

6. Nieuwdorp M, Meuwese MC, Vink H, Hoekstra JB, Kastelein JJ, Stroes ES. The endothelial glycocalyx: a potential barrier between health and vascular disease. Curr Opin Lipidol. 2005;16:507-511.

7. Vink H, Duling BR. Identification of distinct luminal domains for macromolecules, erythrocytes, and leukocytes within mammalian capillaries. Circ Res. 1996;79:581-589.

8. van den Berg BM, Spaan JA, Rolf TM, Vink H. Atherogenic region and diet diminish glycocalyx dimension and increase intima-to-media ratios at murine carotid artery bifurcation. Am J Physiol Heart Circ Physiol. 2006;290:H915-920.

9. Rostgaard J, Qvortrup K. Sieve plugs in fenestrae of glomerular capillaries--site of the filtration barrier? Cells Tissues Organs. 2002;170:132-138.

10. Hjalmarsson C, Johansson BR, Haraldsson B. Electron microscopic evaluation of the endothelial surface layer of glomerular capillaries. Microvasc Res. 2004;67:9-17.

11. Rehm M, Zahler S, Lotsch M, Welsch U, Conzen P, Jacob M, Becker BF. Endothelial glycocalyx as an additional barrier determining extravasation of $6 \%$ hydroxyethyl starch or $5 \%$ albumin solutions in the coronary vascular bed. Anesthesiology. 2004;100:1211-1223.

12. van den Berg BM, Spaan JA, Vink H. Impaired glycocalyx barrier properties contribute to enhanced intimal low-density lipoprotein accumulation at the carotid artery bifurcation in mice. Pflugers Arch. 2009;457:1199-1206.

13. Megens RT, Reitsma S, Schiffers PH, Hilgers RH, De Mey JG, Slaaf DW, oude Egbrink MG, van Zandvoort MA. Two-photon microscopy of vital murine elastic and muscular arteries. Combined structural and functional imaging with subcellular resolution. J Vasc Res. 2007;44:87-98.

14. Megens RT, oude Egbrink MG, Merkx M, Slaaf DW, van Zandvoort MA. Two-photon microscopy on vital carotid arteries: imaging the relationship between collagen and inflammatory cells in atherosclerotic plaques. J Biomed Opt. 2008;13:044022.

15. Thurston G, Baluk P, Hirata A, McDonald DM. Permeability-related changes revealed at endothelial cell borders in inflamed venules by lectin binding. Am J Physiol. 1996;271:H2547-2562.

16. Henry CB, DeFouw DO. Differential lectin binding to microvascular endothelial glycoconjugates during normal angiogenesis in the chick chorioallantoic membrane. Microvasc Res. 1995;49:201211.

17. Meyer W, Godynicki S, Tsukise A. Lectin histochemistry of the endothelium of blood vessels in the mammalian integument, with remarks on the endothelial glycocalyx and blood vessel system nomenclature. Ann Anat. 2008;190:264-276.

18. Accili D, Menghi G, Gabrielli MG. Lectin histochemistry for in situ profiling of rat colon sialoglycoconjugates. Histol Histopathol. 2008;23:863-875. 
19. Zarins CK, Giddens DP, Bharadvaj BK, Sottiurai VS, Mabon RF, Glagov S. Carotid bifurcation atherosclerosis. Quantitative correlation of plaque localization with flow velocity profiles and wall shear stress. Circ Res. 1983;53:502-514.

20. van Triest HJ, Megens RT, van Assen HC, ter Haar Romeny BM, van Zandvoort MA. Arterial radius estimation from microscopic data using a new algorithm for circle parameter estimation. $J$ Biomed Opt;15:026012.

21. Barker AL, Konopatskaya O, Neal CR, Macpherson JV, Whatmore JL, Winlove CP, Unwin PR, Shore AC. Observation and characterisation of the glycocalyx of viable human endothelial cells using confocal laser scanning microscopy. Phys Chem Chem Phys. 2004;6:1006-1011.

22. Twisk JWR. Applied multilevel analysis: a practical guide. Cambridge, UK; New York: Cambridge University Press; 2006.

23. Baldwin AL, Winlove CP. Effects of perfusate composition on binding of ruthenium red and gold colloid to glycocalyx of rabbit aortic endothelium. J Histochem Cytochem. 1984;32:259-266.

24. Megens RT, Oude Egbrink MG, Cleutjens JP, Kuijpers MJ, Schiffers PH, Merkx M, Slaaf DW, van Zandvoort MA. Imaging collagen in intact viable healthy and atherosclerotic arteries using fluorescently labeled CNA35 and two-photon laser scanning microscopy. Mol Imaging. 2007;6:247-260. 


\section{Endothelial glycocalyx thickness}

\section{and platelet-vessel wall}

interactions during

atherogenesis

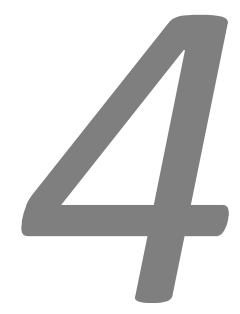

Published as

Sietze Reitsma, Mirjam G.A. oude Egbrink, Viviane V.Th. Heijnen, Remco T.A. Megens, Wim Engels, Hans Vink, Dick W. Slaaf, Marc A.M.J. van Zandvoort 


\section{Abstract}

The endothelial glycocalyx (EG), the luminal cover of endothelial cells, is considered to be atheroprotective. During atherogenesis, platelets adhere to the vessel wall, possibly triggered by simultaneous EG modulation. It was the objective of this study to investigate both EG thickness and platelet-vessel wall interactions during atherogenesis in the same experimental model. Intravital fluorescence microscopy was used to study platelet-vessel wall interactions in vivo in common carotid arteries and bifurcations of $\mathrm{C} 57 \mathrm{bl6} / \mathrm{J}$ (B6) and apolipoprotein $\mathrm{E}$ knock-out (ApoE ${ }^{-/}$) mice (age 7 - 31 weeks). At the same locations, EG thickness was determined ex vivo using two-photon laser scanning microscopy. In $\mathrm{ApoE}^{-/-}$bifurcations the overall median level of adhesion was 48 platelets $/ \mathrm{mm}^{2}$ (interquartile range: $16-80$ ), which was significantly higher than in $\mathrm{B} 6$ bifurcations $(0(0-16), p=0.001)$. This difference appeared to result from a significant age-dependent increase in $\mathrm{ApoE}^{-/}$ mice, while no such change was observed in B6 mice. At the same time, the EG in $\mathrm{ApoE}^{-/-}$bifurcations was significantly thinner than in B6 bifurcations (2.2 vs. $2.5 \mu \mathrm{m}$, respectively; $p<0.05)$. This resulted from the fact that in $B 6$ bifurcations $E G$ thickness increased with age (from $2.4 \mu \mathrm{m}$ in young mice to $3.0 \mu \mathrm{m}$ in aged ones), while in bifurcations of $\mathrm{ApoE}^{-/-}$mice this growth appeared to be absent $(2.2 \mu \mathrm{m}$ at all ages). During atherogenesis, platelet adhesion to the wall of the carotid artery bifurcation increases significantly. At the same location, EG growth with age is hampered. Therefore, glycocalyx-reinforcing strategies could possibly ameliorate atherosclerosis. 


\section{Introduction}

The endothelial glycocalyx (EG) is the carbohydrate-rich luminal lining of endothelial cells, that functions as barrier between blood and endothelium. ${ }^{1}$ The EG is proposed to have vasculoprotective properties, based on its capacity to prevent blood cell-vessel wall interactions, to reduce oxidative stress via incorporated enzymes, and to contribute to the vascular permeability barrier. ${ }^{1-4}$ Furthermore, EG disruption has been associated with vascular disease or endothelial dysfunction, such as occurs in diabetes ${ }^{5}$ or ischemia/reperfusion. ${ }^{6}$ The role of the EG in atherogenesis (i.e., the development of atherosclerotic lesions in the vessel wall) is not well understood. In mouse carotid arteries, atherogenic regions are associated with a reduction of EG thickness ${ }^{7}$ and loss of vasculoprotective properties of the endothelium, as intimal low density lipoprotein (LDL) accumulation is increased in areas with a thinner EG. ${ }^{8}$

Atherosclerosis is an inflammatory process, marked by the formation of lesions in the vessel wall at predilection sites under conditions of high LDL concentrations and disturbed flow patterns. ${ }^{9,} 10$ Platelets play a pivotal role during early stages of atherogenesis. In apolipoprotein $\mathrm{E}$ knock-out $\left(\mathrm{ApoE}^{-/-}\right)$mice fed a high fat diet, an increasing number of platelets was observed to interact with the vessel wall of the carotid artery bifurcation, prior to the occurrence of leukocyte adhesion and lesion formation. Chronic inhibition of platelet glycoprotein Ib $\alpha$ prevented the formation of atherosclerotic plaques in this model. ${ }^{11}$ In addition, activated platelets have been shown to interact with the endothelium at lesion-prone sites in $\mathrm{ApoE}^{-/-}$mice, exacerbating atherogenesis via delivery of various chemokines, such as CCL5 (RANTES) and CXCL4 (platelet factor 4). ${ }^{12-17}$

The initial trigger for platelet adhesion and subsequent chemokine release in the course of atherogenesis has not yet been identified. From literature, a number of findings indicate that the EG might contribute to this process: (1) Chronic inhibition of hyaluronan synthesis (an important component of the EG) accelerated atherosclerotic plaque formation in $\mathrm{ApoE}^{-/-}$mice, and led to a prothrombotic state not caused by increased platelet activation. EG dimensions were reduced in these mice. $^{18}$ (2) Oxidized LDL-mediated degradation of the EG has been shown to result in an increase of platelet-endothelium interactions in hamster cremaster capillaries. $^{19}$ (3) In hyperlipidemic mice the dimensions of the EG are reduced, especially at atherogenesis-prone vascular sites. ${ }^{7,8}$ However, no study has yet determined plate- 
let adhesion as well as EG dimensions at an atherogenic arterial bifurcation in the same experimental model.

The aim of the present study was to assess platelet-vessel wall interactions and EG dimensions during atherogenesis in the carotid bifurcation (lesion-prone site) as compared to the common carotid artery (lesion-protected site) in $\mathrm{ApoE}^{-/-}$mice; C57bl6/J mice were used as wild-type controls. To this purpose, we combined intravital fluorescence microscopy (IFM) to quantify platelet-vessel wall interactions, ${ }^{20,21}$ with our recently developed ex vivo method of EG visualization and quantification in carotid arteries of mice using two-photon laser scanning microscopy (TPLSM). ${ }^{22}$ We hypothesized that a reduction of EG thickness and an increase in platelet-vessel wall interactions during atherogenesis at lesion-prone sites occur simultaneously, which would suggest a causal relation between both.

\section{Materials and Methods}

All experiments conducted were in line with institutional guidelines and approved by the local ethics committee on the use of laboratory animals. Studies were performed on male C57bl6/J (B6; $n=56)$ and C57bl6/J ApoE $E^{-/-}$mice $\left(\mathrm{ApoE}^{-/-} ; n=53\right.$; Charles River, Maastricht, the Netherlands) aged $7-31$ weeks. Mice were fed a normal chow to prevent accelerated atherogenesis, enabling detailed studies of changes in platelet behavior and EG structure as a function of time and vessel location.

\section{Surgery}

To anesthetize the mice, a mixture of $75 \mathrm{mg} / \mathrm{kg}$ ketamin (Nimatek, Eurovet, Cuijk, the Netherlands) and $15 \mathrm{mg} / \mathrm{kg}$ xylazin (Xylalin, CEVA SANTE Animale BV, Naaldwijk, the Netherlands) was prepared in saline, and injected subcutaneously in a volume of $3 \mathrm{ml} / \mathrm{kg}$. To maintain anesthesia smaller doses of $0.88 \mathrm{ml} / \mathrm{kg}$ were administered every $30 \mathrm{~min}$. Body temperature was monitored and maintained at $37^{\circ} \mathrm{C}$ using a heating stage (TH60-SMZ, Linkam Scientific Instruments, Tadworth, UK). The left jugular vein was cannulated (PE 10, Sims Portex, London, UK) for administration of fluorescent dyes. The right carotid artery was exposed up to the bifurcation and a small piece of black plastic was placed underneath the distal part of the common carotid artery and the bifurcation to reduce background noise during fluorescence microscopy. 


\section{Intravital fluorescence microscopy (IFM)}

IFM was used to visualize spontaneous platelet-vessel wall interactions in the right common carotid artery as well as in the carotid bifurcation. The left carotid was used in case the right carotid bifurcation could not be visualized due to anatomical variation. Circulating blood platelets were labeled fluorescently by intravenous administration of $0.03 \mathrm{ml}$ acridin red solution $(A R, 2.0 \mathrm{mg} / \mathrm{ml}$ in saline with $5 \%$ ethanol, Chroma-Gesellschaft Schmid GmbH, Germany). The intravital microscope (Leitz, Wetzlar, Germany) was equipped with an objective for overview recordings (UO 6.5×, numerical aperture 0.18), a water immersion objective (SW 25×, numerical aperture 0.60$)$, a $1.25 \times$ ploemopak for interchangeable filter sets, and a $100 \mathrm{~W}$ mercury lamp. To ensure similar light exposure in each experiment, the yield of the mercury lamp was measured every experiment.

To assess the diameter of the common carotid artery, incident illumination brightfield microscopy recordings were made with the UO 6.5× lens and a POL-cube (polarizer, 50\% mirror, and a crossed analyzer in the imaging pathway) to cancel direct reflections. ${ }^{23}$ To visualize spontaneously interacting fluorescently labeled platelets, a N2-1 filter set (excitation 515 - $560 \mathrm{~nm}$; dichroic $580 \mathrm{~nm}$; barrier $580 \mathrm{~nm}$ longpass) was applied in combination with the SW $25 \times$ water dipping objective. Fluorescence microscopy was applied on the common carotid artery and subsequently on the bifurcation just upstream of the flow divider region, with a distance of at least $500 \mu \mathrm{m}$ between the two sites (Figure 4.1a). Due to movement of the artery and scattering of light, stable images could only be obtained from the upper segment of the exposed vessel (Figure 4.1b). The imaged area (approximately $250 \times$ $250 \mu^{2}$ ) was captured at 30 frames/s into $1000 \times 1000$ pixels for 2.5 min per site using an EM-CCD camera (9100-2, Hamamatsu Photonics, Herrsching am Ammersee, Germany).

\section{Plasma analysis}

After intravital recordings, mice were sacrificed. Blood was collected in $0.1 \mathrm{ml}$ $0.129 \mathrm{M}$ citrate through puncture of the right ventricle. Plasma was isolated $\left(13,000 \mathrm{rpm}\right.$ for $5 \mathrm{~min}$ at $\left.4{ }^{\circ} \mathrm{C}\right)$ and stored at $-80^{\circ} \mathrm{C}$ for later analysis. Plasma levels of high density lipoprotein (HDL), triglycerides, and total cholesterol were assessed using a Beckman/Coulter Synchron LX20 plasma analyzer. LDL concentration was calculated using the Friedewald formula. 


\section{Isolation and mounting of carotid arteries}

For TPLSM imaging of the EG, carotid arteries were processed as previously described. $^{22,24,25}$ In short, both carotid arteries including the bifurcation were carefully dissected, excised, and mounted on two glass micropipettes in a homebuilt perfusion chamber (IDEE, Maastricht, the Netherlands). ${ }^{24}$ Fluorescent labeling of vascular cell nuclei and the EG was achieved by perfusing the artery with $500 \mu \mathrm{l}$ phosphate buffered saline (PBS, pH 7.4) containing $5 \mu \mathrm{M}$ SYTO41 (Molecular Probes, Leiden, the Netherlands) and $2.6 \mu \mathrm{M}$ fluorescein isothiocyanate-labeled wheat germ agglutinin (WGA-FITC; Sigma Aldrich, Zwijndrecht, the Netherlands) at a rate of $1 \mathrm{ml} / \mathrm{h}$ for $30 \mathrm{~min}$, which results in an estimated low wall shear stress of $0.02 \mathrm{~Pa}$. WGA-FITC binds sialic acids and also glucosamine-based glycosaminoglycans such as heparan sulfate and hyaluronan, providing general labeling of the EG. $^{22}$ In carotids from six ApoE ${ }^{-/-}$and two B6 mice, phycoerythrin-conjugated rat anti-mouse CD11b antibody (anti-CD11b-PE; final concentration $8 \mu \mathrm{g} / \mathrm{ml}$; BD Biosciences, Alphen aan den Rijn, the Netherlands) was added to the labeling mixture as a marker for inflammatory cell migration to the subendothelium. After labeling, a transmural pressure of $40-80 \mathrm{mmHg}$ was applied using a modified sphygmomanometer (Big Ben, Riester, Jungingen, Germany). Ex vivo vessel processing and labeling was performed at room temperature.

\section{Two-photon laser scanning microscopy (TPLSM)}

The perfusion chamber was positioned on a Nikon E600FN microscope (Nikon, Tokyo, Japan), coupled to a standard BioRad 2100 MP multiphoton system (Bio-Rad, Hemel Hempstead, UK). A $120 \pm 20$-fs-pulsed Ti:Sapphire laser (Spectra Physics Tsunami, Mountain View, Calif., USA) tuned and mode-locked at $800 \mathrm{~nm}$ was used as excitation source. A 60× water dipping objective (Nikon; numerical aperture 1.0, working distance $2 \mathrm{~mm}$ ) was used and the resulting maximum field of view was approximately $200 \times 200 \mu^{2}$. If necessary, optical zoom could be applied to enhance cellular details (e.g., presence of CD11b-positive cells, Figure 4.1c). The fluorescent signals from SYTO41, WGA-FITC, and anti-CD11b-PE were detected by three photo multiplier tubes (PMTs); PMT1 (pseudo-color blue) received the SYTO41 signal at $420-470 \mathrm{~nm}$; PMT2 (pseudo-color green) received the WGA-FITC signal at $510-550 \mathrm{~nm}$; PMT3 (pseudo-color red) received the anti-CD11b-PE signal at $570-$ $640 \mathrm{~nm}$. Single images ( $512 \times 512$ pixels) were recorded in the xy plane and subsequently collected at successive depths ( $z$ stack; distance between the xy planes was 0.45 or $0.6 \mu \mathrm{m})$. TPLSM and IFM recordings were made at approximately the same locations (common carotid artery and bifurcation; Figures $4.1 \mathrm{a}$ and b). 
a COMMON BIFURCATION
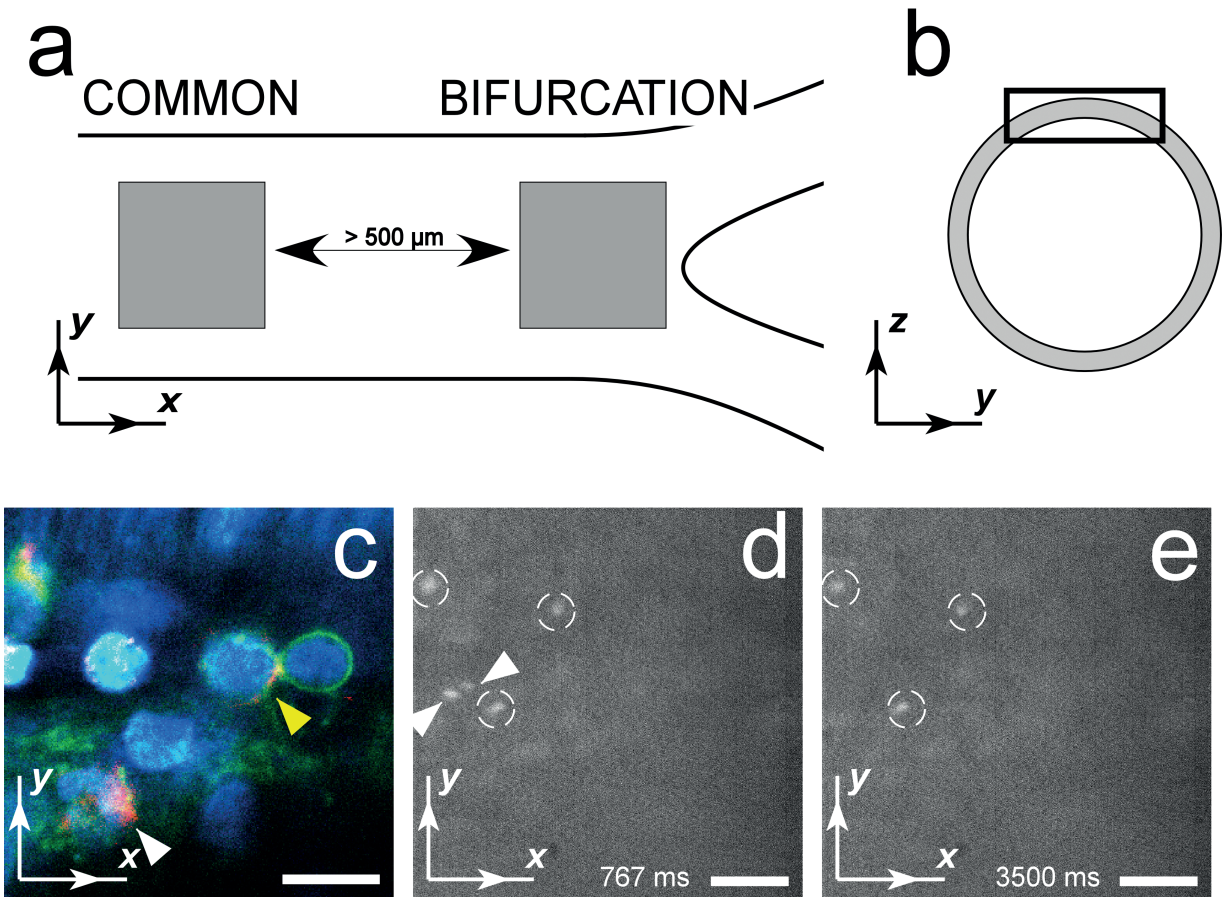

Figure 4.1 Imaging locations.

Schematic field of view and scanning locations in longitudinal $(a)$ and transversal $(b)$ direction in the surgically exposed (IFM) or mounted (TPLSM) carotid artery. Recordings were made in the common carotid artery and in the carotid bifurcation. The distance between these sites was at least $500 \mu \mathrm{m}$. IFM was performed with a field of view of about $250 \times 250 \mu \mathrm{m}^{2}$, while that of TPLSM images was about $200 \times$ $200 \mathrm{\mu m}^{2}$. Both imaging modalities were applied to the top of the vessel, as depicted by the black rectangle in $b$.

c Optical section obtained using two-photon laser scanning microscopy ex vivo in the right carotid artery bifurcation of an 18-week-old ApoE ${ }^{-/}$mouse labeled with SYTO41 (blue, cell nuclei), WGA-FITC (green, endothelial glycocalyx) and anti-CD11b-PE (red, CD11b-positive cells). CD11b-positive cells (arrowheads) were found under the endothelium. The nuclear shape hints at their differentiation; white arrowhead polymorphonuclear granulocyte; yellow arrowhead monocyte. Note the WGA labeling (green) of the membrane of these cells as well. Bar represents $10 \mu \mathrm{m}$.

$d$ Using IFM, both adhering (circles) and saltating (arrowheads) platelets could be observed in the carotid bifurcation of a 25 -week-old $\mathrm{ApoE}^{-/-}$mouse. Saltating platelets only interacted for a short period of time, while the adhering platelets stayed at one site for more than $20 \mathrm{~s}$. $e$ The same vessel $2.7 \mathrm{~s}$ later. Both saltating platelets (arrowheads in $d$ ) are now gone, whereas the adhering ones are still present. Bars represent $50 \mu \mathrm{m}$. Recordings were made at 30 frames/s. Numbers indicate the time (in ms) from the start of the recording.

\section{Data processing}

IFM video analysis was performed offline with the Wasabi software package (version 1.5, Hamamatsu Photonics), allowing quantification of vessel diameter and frame-by-frame monitoring of platelet-vessel wall interactions. Two categories of 
the latter were identified: saltating and adhering platelets. Platelets that attached to the vessel wall at a specific site for at least $90 \mathrm{~ms}$ (4 successive images) and subsequently detached were defined as saltating and expressed as number of saltating platelets $/ \mathrm{mm}^{2} / \mathrm{min}$. Platelets that adhered to the same spot of the vessel wall for longer than $20 \mathrm{~s}$ were defined as adhering. Numbers are expressed as number of adhering platelets $/ \mathrm{mm}^{2}$. For examples of each category, see Figures $4.1 \mathrm{~d}$ and e.

Saltating platelets were found throughout each recording, while most adhering platelets were usually seen from the beginning onwards. Therefore, numbers of adhering platelets are expressed without time scale.Heart rates were estimated from repetitive vessel wall movements in the video stream. During IFM recording, almost no leukocytes were found interacting with the vessel wall in either strain or age group. In those few occurrences, leukocytes could be discriminated from platelets by their size and fluorescence intensity. Data from IFM experiments were discarded when arterial movement impaired successful imaging of platelet-vessel wall interactions, when signal-to-noise ratio (SNR) was too low, or when animals died preliminarily. The final number of IFM experiments (common / bifurcation) was for B6: 33 / 24, and for ApoE $^{-/-}: 29$ / 26.

TPLSM data were analyzed with Imagepro software package (version 6.1, Media Cybernetics, Silver Spring, Md., USA) including the three-dimensional reconstructor set (version 5.1). For image computations, Matlab software (version 7.01, The MathWorks Inc., Natick, Mass., USA) was used. Using a recently described image analysis protocol, EG thickness was calculated per scanned vessel segment. ${ }^{22}$ TPLSM data were discarded when adequate mounting of the carotid artery including the bifurcation was not feasible, when WGA-labeling was incomplete, and/or when SNR was too low. ${ }^{22}$ The final number of TPLSM experiments (common / bifurcation) was for B6: $21 / 13$, and for $\mathrm{ApoE}^{-/-}: 25$ / 12. The surface area labeled with WGA-FITC per vessel segment was comparable between these groups (typically $>90 \%)$.

\section{Statistical analysis}

Statistical analyses were performed using the SPSS software package (version 15.0, SPSS Inc., Chicago, III., USA). Data are presented as median (interquartile range) unless otherwise specified. Age and/or strain groups were compared using the nonparametric Mann-Whitney test. Nonparametric testing was used either because of group sizes (TPLSM data) or for reasons of skewed data distribution (IFM data). The level of significance was set at 0.05 . 


\section{Results}

\section{Influence of strain and vessel site on platelet adhesion and EG thickness}

The average number of adhering platelets in the carotid bifurcation of all $\mathrm{ApoE}^{-/-}$ mice together was significantly higher than in all B6 bifurcations (median: 48 (interquartile range: $16-80$ ) platelets $/ \mathrm{mm}^{2}$ vs. $0(0-16), p=0.001$; Figure 4.2, upper left panel). At the same time, $E G$ thickness in the carotid bifurcation of $A p o E^{-/-}$mice was significantly lower $\left(2.2(2.1-2.4) \mu \mathrm{m}\right.$ in $\mathrm{ApoE}^{-/-}$vs. $2.5(2.3-3.0)$ in B6; $\mathrm{p}<$ 0.05 ; Figure 4.2, upper right panel).

No strain difference was observed in the number of adhering platelets in the common carotid artery $\left(\mathrm{ApoE}^{-/-} 0(0-32)\right.$ platelets $/ \mathrm{mm}^{2}$ vs. B6 $0(0-16)$; Figure 4.2, lower left panel). In parallel, EG thickness in the common carotid was comparable between both strains $\left(1.9(1.7-2.3) \mu \mathrm{m}\right.$ in $\mathrm{ApoE}^{-/-}$vs. $2.2(1.8-2.5)$ in B6; Figure 4.2 , lower right panel). In a subgroup of experiments, both IFM and TPLSM data were available from the same subjects. In these few cases $\left(A p o E^{-1-}: n=5 ; B 6: n=4\right)$, an inverse relation between EG thickness and the number of adhering platelets was observed (not shown). The number of saltating platelets was comparable for both strains (common: B6: $45(10-147)$ platelets $/ \mathrm{mm}^{2} / \mathrm{min}, \mathrm{ApoE}^{-/-}$: 96 (29 - 176); bifurcation: B6: 115 (32-186), $\mathrm{ApoE}^{-/-}: 83(0-320)$; not shown).

\section{Influence of age}

As presented in the previous section, a significantly higher number of adhering platelets coincides with lower EG thickness in the bifurcation of $\mathrm{ApoE}^{-/-}$mice as compared to wild-type controls. However, both IFM and TPLSM data show large variation (Figure 4.2). In order to explain (part of) this variation, the effect of age was considered (Figure 4.3). In $\mathrm{ApoE}^{-/-}$mice, the number of adhering platelets in the carotid bifurcation increased with age (solid line in the top right panel of Figure 4.3; Spearman's rho 0.497, $\mathrm{p}=0.01$ ). This resulted in a significant difference between young (7 - 18 weeks) and aged (19- 31 weeks) ApoE ${ }^{-/-}$mice (24 (0 - 52) platelets $/ \mathrm{mm}^{2}$ vs. $\left.64(48-104) ; \mathrm{p}<0.01\right)$.

No age-related changes in the number of adhering platelets were found in B6 bifurcations (Figure 4.3, upper left panel). Conversely, no relation between age and EG thickness was found in the bifurcation of $\mathrm{ApoE}^{-/-}$mice (young: $2.2(2.0-2.7) \mu \mathrm{m}$; aged: $2.2(2.1-2.5) \mu \mathrm{m})$, while EG thickness significantly increased with age in B6 bifurcations (young: $2.4(2.2-2.6) \mu \mathrm{m}$; aged $3.0(2.6-3.1) \mu \mathrm{m} ; \mathrm{p}<0.05$; Figure 4.3, lower panels). 

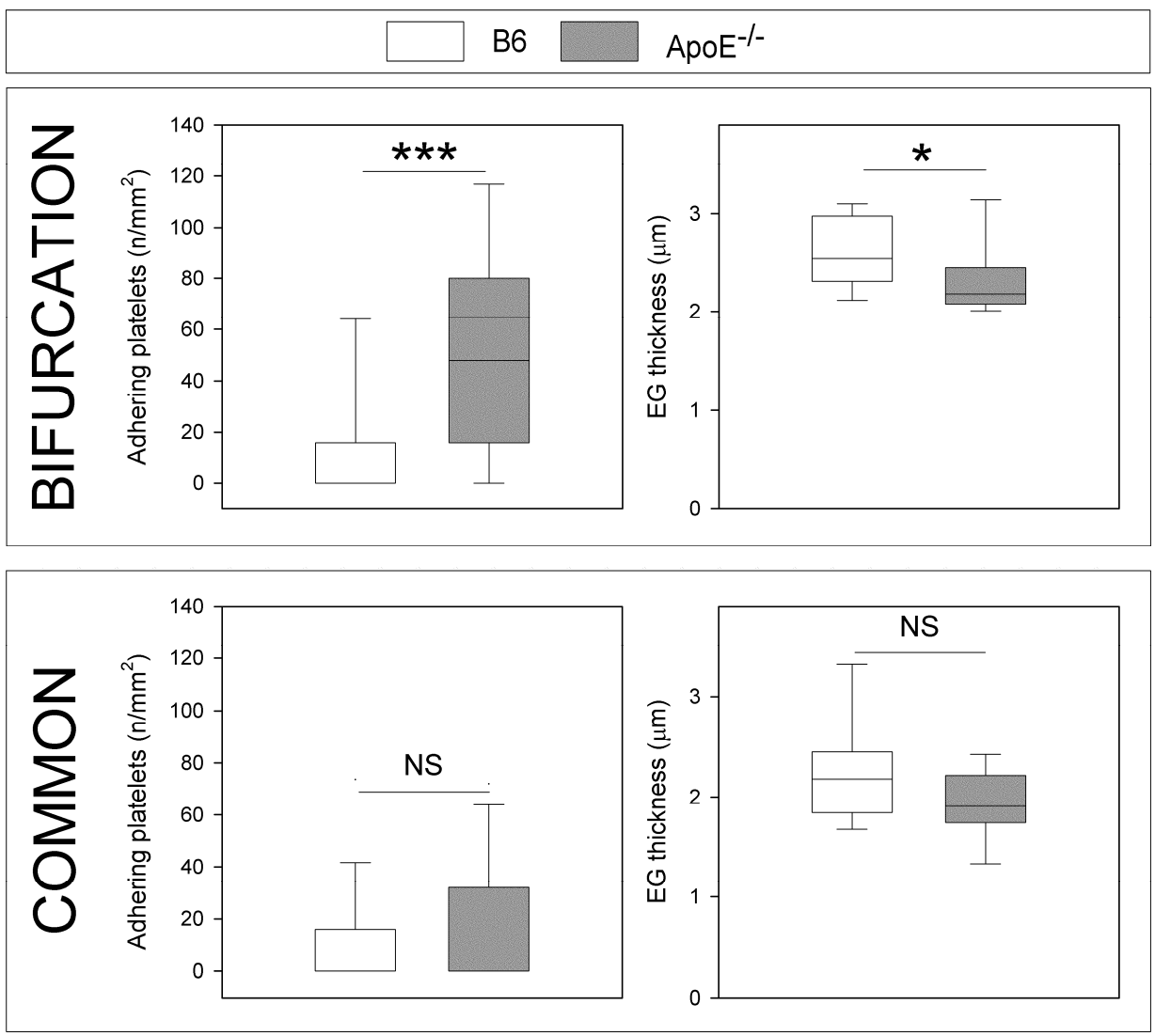

Figure 4.2 Platelet adhesion and EG thickness in the carotid artery of B6 and ApoE ${ }^{-/-}$mice.

Top panels Number of adhering platelets (left) and EG thickness (right) in the carotid bifurcation of B6 and $\mathrm{ApoE}^{-/-}$mice.

Lower panels Same parameters in the common carotid artery of both strains.

Data are presented as box and whisker plots showing the $10^{\text {th }}, 25^{\text {th }}, 50^{\text {th }}, 75^{\text {th }}$ and $90^{\text {th }}$ percentiles. Outliers are not depicted. ${ }^{*} \mathrm{p} \leq 0.05 ; * * \mathrm{p} \leq 0.001 ;$ NS not significant. 


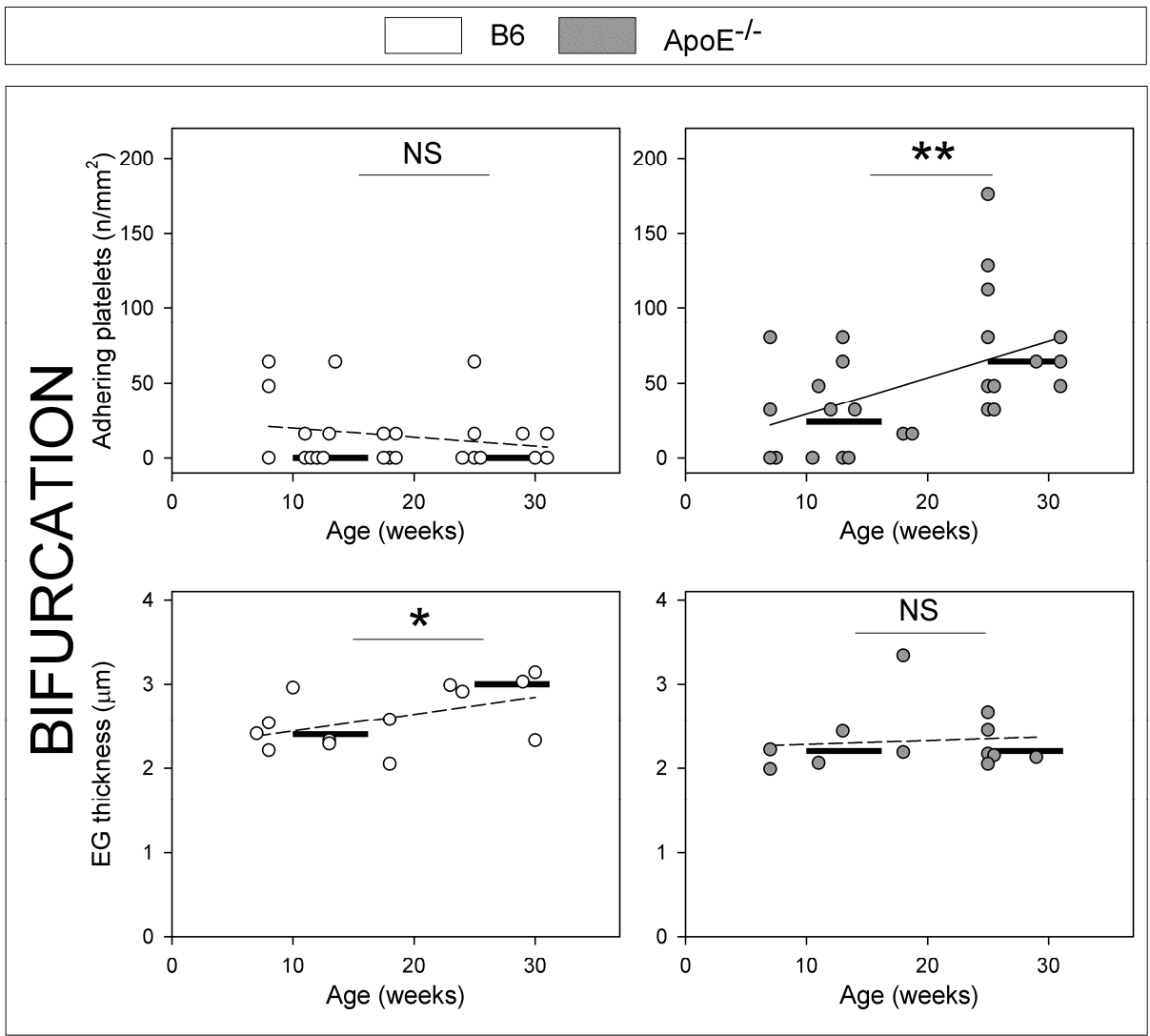

Figure 4.3 Influence of age on platelet adhesion and EG thickness in carotid bifurcations of B6 and $\mathrm{ApoE}^{-/}$mice.

Top panels Relation between age and the number of adhering platelets in the carotid bifurcation of B6 (left) and $\mathrm{ApoE}^{-/-}$mice (right).

Lower panels Relation between age and EG thickness in the carotid bifurcation of B6 (left) and ApoE ${ }^{-/-}$ mice (right).

Data are presented as actual data points. Medians of age groups (young: 7 - 18, and aged: 19 - 31 weeks) are depicted as black, horizontal bars. Lines indicate correlation between age and adhering platelets or EG thickness. Dotted lines no correlation; solid line significant correlation. * $p \leq 0.05$; ${ }^{* *} p \leq$ 0.01 ; NS not significant 
Table 4.1 General characteristics of the mouse groups used

\begin{tabular}{|c|c|c|c|c|}
\hline & Young & & Aged & \\
\hline & B6 & $\mathrm{ApoE}^{-/-}$ & B6 & $\mathrm{ApoE}^{-/-}$ \\
\hline Weight (g) & $26 \pm 2.1$ & $27 \pm 3.5$ & $28 \pm 1.3^{\dagger}$ & $32 \pm 2.4^{*+\S}$ \\
\hline $\begin{array}{l}\text { Plasma } \\
\text { cholesterol (mM) }\end{array}$ & $2.0 \pm 0.91$ & $9.4 \pm 1.61^{*+}$ & $1.8 \pm 0.35$ & $10.7 \pm 2.67^{*+}$ \\
\hline Plasma LDL (mM) & $0.2 \pm 0.18$ & $6.3 \pm 1.12^{*+}$ & $0.1 \pm 0.08$ & $7.6 \pm 1.89^{*+\S}$ \\
\hline Heart rate (bpm) & $255 \pm 28$ & $282 \pm 28$ & $253 \pm 42$ & $272 \pm 49$ \\
\hline CCA diameter $(\mu \mathrm{m})$ & $454 \pm 41$ & $460 \pm 52$ & $445 \pm 48$ & $452 \pm 38$ \\
\hline
\end{tabular}

B6 wild-type C57bl6/J mice; $A p o E^{-1-}$ Apolipoprotein E knock-out mice; young 7-18 weeks; aged 19-31 weeks; $L D L$ low density lipoprotein; CCA common carotid artery. Data are presented as mean \pm standard deviation. $\mathrm{N}$-values differ per cell as not all measurements were performed in all animals. Groups were compared using one-way ANOVA with Bonferroni correction. ${ }^{*} p \leq 0.05$ compared to aged $\mathrm{B} 6 ;{ }^{\dagger} \mathrm{p} \leq 0.05$ compared to young $\mathrm{B} 6 ;^{\S} \mathrm{p} \leq 0.05$ compared to young $\mathrm{ApoE}^{-/-}$

\section{Atherogenesis}

General characteristics of $\mathrm{B} 6$ and $\mathrm{ApoE}^{-/-}$mice used are listed in Table 4.1. Already at seven weeks, $\mathrm{ApoE}^{-/-}$mice showed hypercholesterolemia and increased levels of $\mathrm{LDL}$ compared to $\mathrm{B} 6$. In the carotid artery bifurcation of $\mathrm{ApoE}^{-/-}$mice, subendothelial CD11b-positive inflammatory cells were found from the age of 18 weeks onward (Figure 4.1c), while plaques were macroscopically visible at the age of 25 weeks and older. In carotid arteries of B6 mice, neither plaques nor CD11b-positive cells were found, as in the common carotids of $\mathrm{ApoE}^{-/-}$mice (not shown).

\section{Discussion}

The current study was employed to investigate platelet-vessel wall interactions and EG dimensions during atherogenesis in the same experimental model. To this end, two different microscopy techniques were combined. Data obtained in this way show that the level of platelet adhesion in the carotid bifurcation of $\mathrm{ApoE}^{-/-}$mice is significantly higher, while at the same time the EG at this location is thinner as compared to wild-type controls. This difference in EG dimension between ApoE ${ }^{-/-}$ and control bifurcations appears to be due to an absence of growth of the EG in the hyperlipidemic animals, while EG thickness increases with age in the controls.

During early atherogenesis, a combination of factors initiates platelet adhesion to the vessel wall, which in turn contributes to local inflammation and subsequent formation of plaques. ${ }^{11,13-15}$ The findings of this study indicate that: (a) adhering 
platelets are mainly found in $\mathrm{ApoE}^{-/-}$mice (effect of mouse strain; Figure 4.2); (b) in the carotid bifurcation, significantly more platelets adhere to the wall than in the common carotid artery (effect of vascular location; Figure 4.2); and (c) the level of platelet adhesion in the carotid bifurcation of $\mathrm{ApoE}^{-/-}$mice increases significantly with age (effect of age; Figure 4.3 upper right panel).

In B6 mice, no age-dependent increase in platelet adhesion is found. At the same time, B6 mice show an age-dependent growth of EG, that is not observed in $\mathrm{ApoE}^{-/-}$ mice (Figure 4.3). These findings suggest that a lack of EG growth with age in $\mathrm{ApoE}^{-/-}$mice may have contributed to the increase in platelet adhesion at the carotid bifurcation (effect of glycocalyx, Figure 4.3). However, the absolute dimension of the $\mathrm{EG}$ in the bifurcation of $\mathrm{ApoE}^{-/-}$mice is comparable to that of the common carotid artery in the same animals (Figure 4.2), while the level of platelet adhesion differs significantly between both sites. This contradicts a simple and straightforward inverse relation between EG thickness and level of platelet adhesion. We suggest that smaller EG dimensions are not the sole determinant, but that they combine with other site-specific factors, such as flow profiles, to explain the agedependent increase in platelet adhesion at the carotid bifurcation of $\mathrm{ApoE}^{-/-}$mice. In line with this hypothesis, EG reinforcing strategies ${ }^{26}$ could help in the prevention of atherosclerosis.

Our data are in line with other publications showing that platelets adhere to atherogenesis-prone sites prior to lesion formation and that damage to the EG further stimulates the formation of atherosclerotic plaques. ${ }^{11,13,18}$ In support of this view, we report a thinner $E G$ in the carotid bifurcation of $\mathrm{ApoE}^{-/-}$mice than of wild-type controls. In the carotid bifurcation of $A p o E^{-/-}$mice, the overall median EG thickness was found to be $2.2 \mu \mathrm{m}$. Although significantly lower than in control bifurcations, this EG thickness still largely exceeds the size of adhesion molecules expressed on the endothelium, such as P-selectin (about $0.05 \mu \mathrm{m}$ ). ${ }^{27,28}$ The question arises how platelets can interact with the endothelium despite such a large barrier (even if thinner than in control mice). One explanation may be that platelets have the ability to form very thin membrane extrusions known as tethers, which can reach a length of more than $1 \mu \mathrm{m} .{ }^{29}$ Because the EG is estimated to have a low stiffness, $^{30}$ platelet tethers may easily protrude into the EG to overcome this large obstacle. Other studies describe an increase of EG permeability in the carotid bifurcation of $\mathrm{ApoE}^{-/-}$mice, ${ }^{8}$ which might further facilitate platelet tether protrusion into the EG. On the other hand, EG thicknesses as presented in this study represent a central measure derived from a heterogeneous thickness distribution per vessel segment. ${ }^{22}$ This measure is relatively insensitive to minor changes in local EG com- 
position. In other words, the scanned vessel segments were found to be covered by an EG layer of rather variable thicknesses. Therefore, platelets may not have to overcome the entire reported EG thickness of several micrometers, but may preferentially adhere to endothelial sites covered with thinner EG. Due to the processing of the vessels for ex vivo TPLSM, adhering platelets were not retrieved in the TPLSM images. As a consequence, the relation between the site of platelet adhesion and local EG dimensions is not known. The use of other lectins to label the EG for microscopic imaging might contribute to the identification of thin or more permeable sites in the EG.

We were particularly interested in the relation between EG structure and platelet behavior during atherogenesis. To enable detailed investigation of changes in both factors, all mice were fed a normal diet. As a consequence, elevated plasma levels of cholesterol and LDL as well as atherosclerotic plaque formation were found in $\mathrm{ApoE}^{-/-}$mice only. The fact that platelet-vessel wall interactions occurred more often and at younger ages, and that leukocyte-vessel wall interactions were found commonly in studies with Western diet-fed $\mathrm{ApoE}^{-/-}$mice ${ }^{11,13}$ can be ascribed to the accelerated and significantly greater increase in plasma cholesterol levels in these animals. $^{7,31,32}$

\section{Conclusions}

Our data show that in the carotid bifurcation of hyperlipidemic mice platelet-vessel wall interactions increase significantly in the course of atherogenesis. At the same location, EG growth with age is hampered. Glycocalyx-reinforcing strategies could possibly ameliorate atherosclerosis.

\section{Acknowledgements}

This research is supported by NWO grant \#902-16-276 and SenterNovem (BSIK 03033). 


\section{References}

1. Reitsma S, Slaaf DW, Vink H, van Zandvoort MA, Oude Egbrink MG. The endothelial glycocalyx: composition, functions, and visualization. Pflugers Arch. 2007;454:345-359.

2. Gouverneur M, Berg B, Nieuwdorp M, Stroes E, Vink H. Vasculoprotective properties of the endothelial glycocalyx: effects of fluid shear stress. J Intern Med. 2006;259:393-400.

3. Nieuwdorp M, Meuwese MC, Vink H, Hoekstra JB, Kastelein JJ, Stroes ES. The endothelial glycocalyx: a potential barrier between health and vascular disease. Curr Opin Lipidol. 2005;16:507-511.

4. Constantinescu A, Spaan JA, Arkenbout EK, Vink H, Vanteeffelen JW. Degradation of the endothelial glycocalyx is associated with chylomicron leakage in mouse cremaster muscle microcirculation. Thromb Haemost. 2011;105:790-801.

5. Nieuwdorp M, Mooij HL, Kroon J, Atasever B, Spaan JA, Ince C, Holleman F, Diamant M, Heine RJ, Hoekstra JB, Kastelein JJ, Stroes ES, Vink H. Endothelial glycocalyx damage coincides with microalbuminuria in type 1 diabetes. Diabetes. 2006;55:1127-1132.

6. Mulivor AW, Lipowsky HH. Inflammation- and ischemia-induced shedding of venular glycocalyx. Am J Physiol Heart Circ Physiol. 2004;286:H1672-1680.

7. van den Berg BM, Spaan JA, Rolf TM, Vink H. Atherogenic region and diet diminish glycocalyx dimension and increase intima-to-media ratios at murine carotid artery bifurcation. Am J Physiol Heart Circ Physiol. 2006;290:H915-920.

8. van den Berg BM, Spaan JA, Vink H. Impaired glycocalyx barrier properties contribute to enhanced intimal low-density lipoprotein accumulation at the carotid artery bifurcation in mice. Pflugers Arch. 2009;457:1199-1206.

9. Hansson GK. Inflammation, atherosclerosis, and coronary artery disease. $N$ Engl J Med. 2005;352:1685-1695.

10. Libby P. Inflammation in atherosclerosis. Nature. 2002;420:868-874.

11. Massberg S, Brand K, Gruner S, Page S, Muller E, Muller I, Bergmeier W, Richter T, Lorenz M, Konrad I, Nieswandt B, Gawaz M. A critical role of platelet adhesion in the initiation of atherosclerotic lesion formation. J Exp Med. 2002;196:887-896.

12. Sachais BS, Turrentine T, Dawicki McKenna JM, Rux AH, Rader D, Kowalska MA. Elimination of platelet factor 4 (PF4) from platelets reduces atherosclerosis in C57BI/6 and apoE-/- mice. Thromb Haemost. 2007;98:1108-1113.

13. Huo Y, Schober A, Forlow SB, Smith DF, Hyman MC, Jung S, Littman DR, Weber C, Ley K. Circulating activated platelets exacerbate atherosclerosis in mice deficient in apolipoprotein E. Nat Med. 2003;9:61-67.

14. Koenen RR, von Hundelshausen P, Nesmelova IV, Zernecke A, Liehn EA, Sarabi A, Kramp BK, Piccinini AM, Paludan SR, Kowalska MA, Kungl AJ, Hackeng TM, Mayo KH, Weber C. Disrupting functional interactions between platelet chemokines inhibits atherosclerosis in hyperlipidemic mice. Nat Med. 2009;15:97-103.

15. Weber C. Platelets and chemokines in atherosclerosis: partners in crime. Circ Res. 2005;96:612616.

16. Sandberg WJ, Otterdal K, Gullestad L, Halvorsen B, Ragnarsson A, Froland SS, Damas JK, Oie E, Aukrust P, Hansson GK, Yndestad A. The tumour necrosis factor superfamily ligand APRIL (TNFSF13) is released upon platelet activation and expressed in atherosclerosis. Thromb Haemost. 2009;102:704-710.

17. Aidoudi S, Bikfalvi A. Interaction of PF4 (CXCL4) with the vasculature: a role in atherosclerosis and angiogenesis. Thromb Haemost. 2010;104:941-948. 
18. Nagy N, Freudenberger T, Melchior-Becker A, Rock K, Ter Braak M, Jastrow H, Kinzig M, Lucke S, Suvorava T, Kojda G, Weber AA, Sorgel F, Levkau B, Ergun S, Fischer JW. Inhibition of hyaluronan synthesis accelerates murine atherosclerosis: novel insights into the role of hyaluronan synthesis. Circulation. 2011;122:2313-2322.

19. Vink H, Constantinescu AA, Spaan JA. Oxidized lipoproteins degrade the endothelial surface layer: implications for platelet-endothelial cell adhesion. Circulation. 2000;101:1500-1502.

20. Kuijpers MJ, Gilio K, Reitsma S, Nergiz-Unal R, Prinzen L, Heeneman S, Lutgens E, van Zandvoort MA, Nieswandt B, oude Egbrink MG, Heemskerk JW. Complementary roles of platelets and coagulation in thrombus formation on plaques acutely ruptured by targeted ultrasound treatment: a novel intravital model. J Thromb Haemost. 2009;7:152-161.

21. van Gestel MA, Heemskerk JW, Slaaf DW, Heijnen VV, Sage SO, Reneman RS, oude Egbrink MG. Real-time detection of activation patterns in individual platelets during thromboembolism in vivo: differences between thrombus growth and embolus formation. J Vasc Res. 2002;39:534-543.

22. Reitsma S, oude Egbrink MGA, Vink H, van den Berg B, Lima Passos V, Engels W, Slaaf DW, van Zandvoort MAMJ. Endothelial glycocalyx structure in the intact carotid artery: a two-photon laser scanning microscopy study. J Vasc Res. 2011;48:297-306.

23. Slaaf DW, Tangelder GJ, Reneman RS, Jager K, Bollinger A. A versatile incident illuminator for intravital microscopy. Int J Microcirc Clin Exp. 1987;6:391-397.

24. Megens RT, Reitsma S, Schiffers PH, Hilgers RH, De Mey JG, Slaaf DW, oude Egbrink MG, van Zandvoort MA. Two-photon microscopy of vital murine elastic and muscular arteries. Combined structural and functional imaging with subcellular resolution. J Vasc Res. 2007;44:87-98.

25. Megens RT, Oude Egbrink MG, Cleutjens JP, Kuijpers MJ, Schiffers PH, Merkx M, Slaaf DW, van Zandvoort MA. Imaging collagen in intact viable healthy and atherosclerotic arteries using fluorescently labeled CNA35 and two-photon laser scanning microscopy. Mol Imaging. 2007;6:247-260.

26. Becker BF, Chappell D, Bruegger D, Annecke T, Jacob M. Therapeutic strategies targeting the endothelial glycocalyx: acute deficits, but great potential. Cardiovasc Res. 2010;87:300-310.

27. Springer TA. Adhesion receptors of the immune system. Nature. 1990;346:425-434.

28. Becker BF, Chappell D, Jacob M. Endothelial glycocalyx and coronary vascular permeability: the fringe benefit. Basic Res Cardiol. 2010;105:687-701.

29. Maxwell MJ, Westein E, Nesbitt WS, Giuliano S, Dopheide SM, Jackson SP. Identification of a 2stage platelet aggregation process mediating shear-dependent thrombus formation. Blood. 2007;109:566-576.

30. Han YF, Weinbaum S, Spaan JAE, Vink H. Large-deformation analysis of the elastic recoil of fibre layers in a Brinkman medium with application to the endothelial glycocalyx. Journal of Fluid Mechanics. 2006;554:217-235.

31. Meir KS, Leitersdorf E. Atherosclerosis in the apolipoprotein-E-deficient mouse: a decade of progress. Arterioscler Thromb Vasc Biol. 2004;24:1006-1014.

32. Ramos CL, Huo Y, Jung U, Ghosh S, Manka DR, Sarembock IJ, Ley K. Direct demonstration of Pselectin- and VCAM-1-dependent mononuclear cell rolling in early atherosclerotic lesions of apolipoprotein E-deficient mice. Circ Res. 1999;84:1237-1244. 


\title{
Platelet-vessel wall interactions
}

\author{
in the isolated mouse
}

\section{carotid artery}

Roles for flow profile entrance length and endothelial glycocalyx

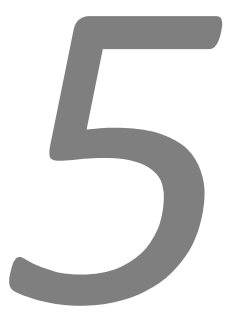

Sietze Reitsma, Marc A.M.J. van Zandvoort, Hans Vink, Dick W. Slaaf, Mirjam G.A. oude Egbrink 


\begin{abstract}
Platelets are involved in atherogenesis. We aimed to assess platelet-vessel wall interactions in isolated large arteries and to study the role of the endothelial glycocalyx (EG) therein. Carotid arteries from 11 mice were isolated, mounted in a vessel flow chamber, and flushed with fluorescently labeled platelets. Platelet-vessel wall interactions were imaged in the common carotid artery and carotid bifurcation using wide-field fluorescence microscopy. Platelet flow pattern was used to determine the entrance length of the developing flow profile. Long entrance lengths induced high levels of platelet pre-activation, which resulted in aggregation and many adhesions (median (interquartile range): common: > 160 (64 - > 160) platelets/mm²; bifurcation: > 160 (44->160)). Vessels with low platelet pre-activation had adhesion levels comparable to in vivo (common: $8(0-36)$; bifurcation 0 ( 0 32)). Enzymatic EG breakdown with hyaluronidase increased adhesion levels to 32 $(24-112 ; p<0.05)$ in the bifurcation, while no effect was seen in the common carotid artery. Conclusively, platelet-vessel wall interactions can be adequately studied in isolated mouse carotid arteries. Vessels with small entrance lengths yield results comparable to in vivo studies. In the carotid bifurcation, EG disruption facilitates platelet adhesion.
\end{abstract}




\section{Introduction}

Platelets are the smallest blood cells and are mainly known for their capacity to form aggregates at sites of vascular damage, and their role in the process of coagulation. Over the past decades, however, it has become increasingly clear that platelets have numerous roles in other (patho)physiological processes as well. ${ }^{1}$ One of the diseases in which platelets play a pivotal role is atherosclerosis, which is an inflammatory disease of the vessel wall of large arteries. ${ }^{2}$ Under conditions of hyperlipidemia, atherosclerotic plaques slowly develop at predilection sites. Rupture of these plaques causes a thromboembolic reaction, ultimately leading to conditions such as myocardial infarction, stroke or peripheral arterial occlusion. As such, atherosclerosis is one of the leading causes of death and morbidity in Western societies. $^{3}$

Platelets are also involved in the early stages of atherogenesis (i.e., the development of atherosclerotic plaques); single platelets adhere to the vessel wall, contributing to the initiation of an inflammatory response. ${ }^{4-7}$ Inhibition of these platelet-vessel wall interactions through long-term adhesion molecule blockade dramatically reduces the development of plaques. ${ }^{7}$ However, chronic inhibition of platelet adhesion with acetylsalicylic acid and/or clopidogrel (both widely used anti-platelet drugs) does not reduce plaque formation, ${ }^{8}$ probably due to other vascular effects (acetylsalicylic acid) or to other preserved platelet adhesion pathways (clopidogrel). Therefore, more insight is needed in the role of platelet adhesion in atherogenesis. To this end, an imaging method is required to investigate platelet behavior at the level of the endothelium of large arteries at adequate temporal and spatial resolution.

Traditionally, in vivo platelet behavior is studied using intravital (fluorescence) microscopy in the microcirculation of thin tissue, such as the mesentery or cremaster muscle. ${ }^{9,} 10$ These tissues are easy to access and relatively motionless, leading to stable images with good optical resolution. Unfortunately, these tissues give a poor impression of disease processes in large arteries. In vivo imaging of platelet-vessel wall interactions in large arteries is challenging. First of all, recordings in these vessels are distorted due to motion artifacts. Secondly, the vessel wall is much thicker, leading to scattering of light and, hence, limited resolution at larger depths. At these depths, the presence of red blood cells further deteriorates image resolution, as they absorb (excitation) light. In addition, platelet-vessel wall interactions can be rapid processes, requiring stable images at short time intervals (tens of milliseconds) for adequate visualization. There are solutions to circumvent (most of) these challenges in large arteries, but they require advanced microscopic techniques 
which are not readily available and lack the needed time resolution. ${ }^{11}$ Several reports describe the use of intravital fluorescence microscopy to assess platelet adhesion and aggregation in the carotid artery of mice. ${ }^{4,5,7,8}$ In our own experience, however, these experiments are challenging and time-consuming, often leading to loss of data due to poor image quality. ${ }^{4}$

As an alternative, platelet studies can be performed in vitro, in specially designed flow chambers, enabling stable imaging of platelet interactions with coated media (e.g., collagen). ${ }^{12,13}$ However, the role of the vessel wall and especially that of the endothelial glycocalyx ${ }^{14}$ cannot be studied accurately in this in vitro setup, even when flow chambers are coated with cultured endothelial cells, as their glycocalyx is not comparable to that of intact vessels in vivo. ${ }^{15}$

Alternatively, mounted arteries can be used. ${ }^{4,16,17}$ This method respects the role of the entire vessel wall in platelet-vessel wall interactions. Several reports describe the use of isolated intact carotid arteries for various purposes, for example to study flow-induced dilation, ${ }^{18}$ or monocyte-vessel wall interactions. ${ }^{19}$ However, no papers were found describing platelet-vessel wall interactions in these arteries.

Therefore, we set up a feasibility study. The aim of this study was threefold: (1) To create a model in which platelet-vessel wall interactions can be studied at adequate temporal and spatial resolution in the intact mounted mouse carotid artery without motion artifacts. (2) To reach platelet adhesion levels that are comparable to the in vivo situation. (3) To study the role of the endothelial glycocalyx in platelet adhesion in these vessels.

During pilot experiments, we noted a large variability in levels of platelet-vessel wall interactions. In some vessels, numbers were low, while in others many interactions could be observed. Furthermore, the flow lines of fluorescent platelets gave an impression of the flow pattern in the mounted vessel, which indicated that the flow profile developed into an axial form with parallel flow lines over a certain part of the proximal common carotid artery (entrance length). ${ }^{20-22}$ Entrance length appeared to vary considerably between experiments and was observed to influence the level of platelet-vessel wall interactions. Therefore, special attention was paid to the developing flow profile and its influence on platelet-vessel wall interactions as it might bias findings regarding the endothelial glycocalyx. 


\section{Methods}

All experiments conducted were in line with institutional guidelines and approved by the local ethics committee on the use of laboratory animals. 22 carotid arteries were sampled from male $\mathrm{C} 57 \mathrm{bl6} / \mathrm{J}$ mice $(\mathrm{B} 6 ; \mathrm{n}=8)$ and male $\mathrm{C} 57 \mathrm{bl6} / \mathrm{J}$ apolipoprotein $\mathrm{E}$ knock-out mice (ApoE $\left.{ }^{-/-} ; n=3\right)$, aged $9-31$ weeks (Charles River, Maastricht, the Netherlands).

\section{Processing of carotid arteries and blood platelets}

Mice were anesthetized using a mixture in saline of $75 \mathrm{mg} / \mathrm{kg}$ ketamin (Nimatek, Eurovet, Cuijk, the Netherlands) and $15 \mathrm{mg} / \mathrm{kg}$ xylazin (Xylalin, CEVA SANTE Animale BV, Naaldwijk, the Netherlands), which was injected subcutaneously in a volume of $3 \mathrm{ml} / \mathrm{kg}$. Via an abdominal approach, the diaphragm was slit and blood was collected in $0.15 \mathrm{ml} 0.129 \mathrm{M}$ citrate through puncture of the right ventricle.

Through centrifugation, a platelet pellet was obtained which was dissolved in 1.5 $\mathrm{ml} 60 \mu \mathrm{M}$ carboxyfluorescein diacetate succinimidyl ester (CFSE; Molecular Probes, Leiden, the Netherlands) in warm phosphate buffered saline (PBS; $35-37^{\circ} \mathrm{C}$ ) containing 0.67 vol\% dimethyl sulfoxide. After $5 \mathrm{~min}$ of incubation at room temperature, platelets were again centrifuged and dissolved in warm PBS. Platelet count was assessed using a Coulter counter (Coulter Electronics). The platelet stock solution was kept in the dark at $35-37^{\circ} \mathrm{C}$ until further use.

The carotid arteries including the bifurcation were isolated and mounted in a vessel perfusion chamber as reported previously. ${ }^{16,17}$ In short, the common and external carotid artery were mounted on a glass micropipette, while the internal carotid artery, as well as some smaller ones, were ligated (Figure 5.1a). A small hair was positioned in the adventitial connective tissue of the common carotid artery and carotid artery bifurcation to mark these locations for repeated measurements.

Arteries were kept in a modified Hank's balanced salt solution (HBSS) containing in $\mathrm{mM}: \mathrm{NaCl} 137, \mathrm{HEPES} 15$, glucose 5.5, $\mathrm{KCl} 5.3, \mathrm{CaCl}_{2} 2.5, \mathrm{Na}_{2} \mathrm{HPO}_{4} 0.34, \mathrm{KH}_{2} \mathrm{PO}_{4}$ 0.44 , and $\mathrm{MgSO}_{4} 1.1\left(\mathrm{pH}=7.4\right.$ at $\left.37^{\circ} \mathrm{C}\right)$. This buffer solution was also used to flush arteries (see below). Some arteries were flushed with an albumin-containing buffer (BSA-SS; $1 \mathrm{mg} / \mathrm{ml}$ bovine serum albumin, and in $\mathrm{mM}$ : $\mathrm{NaCl} 114$, HEPES 5, glucose 5.55, $\mathrm{KCl} 10, \mathrm{CaCl}_{2}$ 0.5, $\mathrm{NaHCO}_{3} 25, \mathrm{KH}_{2} \mathrm{PO}_{4}$ 1.18, EDTA 0.025 and $\mathrm{MgSO}_{4}$ 1.17; $\mathrm{pH}=$ 7.4 at $\left.37^{\circ} \mathrm{C}\right)$. 


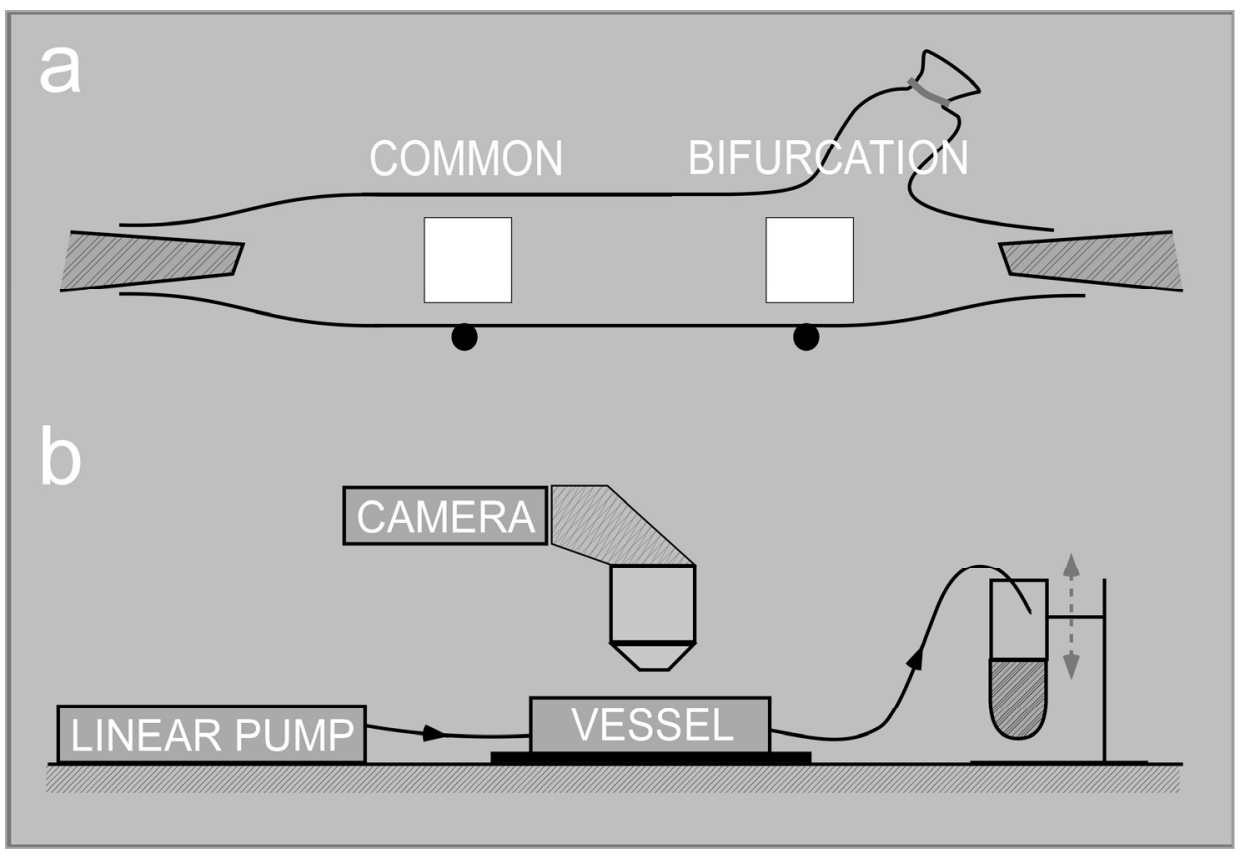

Figure 5.1 Experimental setup.

$a$ Schematic representation of a mounted carotid artery including bifurcation. Note that one side branch is ligated. White boxes represent the microscopy recording areas; images are recorded in the common carotid artery and the bifurcation just upstream of the flow divider region, with the focal plane at the level of the endothelium. Black circles represent small hairs that are positioned in the tunica adventitia as a marker of vessel location. Note that the area of recording does not cover the entire vessel diameter.

$b$ Schematic representation of the microscopy setup. A linear pump is used to flow (platelet-containing) buffer through the mounted vessel. The outflow tract height and, hence, the transmural pressure can be adjusted. The vessel chamber is heated to $35-37{ }^{\circ} \mathrm{C}$ using a heating stage (black bar beneath vessel chamber). The microscope is equipped with an EM-CCD camera for recordings at 32 frames/s.

\section{Microscopy setup}

The vessel chamber was placed under an intravital microscope (Leitz, Wetzlar, Germany) as depicted in Figure 5.1b. The microscope was equipped with an objective for overview recordings (UO 11x, numerical aperture 0.25 ), a water immersion objective (SW 25x, numerical aperture 0.60), a 1.25x Ploemopak for interchangeable filter sets, and a $100 \mathrm{~W}$ mercury lamp. Images were recorded with an EM-CCD camera (9100-2, Hamamatsu Photonics, Herrsching am Ammersee, Germany) at 32 frames per second into $500 \times 500$ pixels. The recording area measured approximately $635 \times 635 \mu \mathrm{m}^{2}$ for overview recordings and $250 \times 250 \mu \mathrm{m}^{2}$ for fluorescence 
recordings, respectively. ${ }^{4}$ The temperature of the vessel chamber was kept at 35 $37^{\circ} \mathrm{C}$ by a heating stage (TH60-SMZ, Linkam Scientific Instruments, England).

\section{Flow and diameter regulation}

Overview recordings were made to measure the vessel outer diameter, while the arteries were flushed using a linear pump. In vivo flow rates in the mouse carotid artery range between $10-50 \mu \mathrm{l} / \mathrm{s} .^{18,23-25}$ Part of the variation in this number can be explained by the anesthetics used; ketamin/xylazin regiments largely influence systemic hemodynamics, leading to lower flow rates. ${ }^{26}$ In order to compare the data from the current model with our in vivo data obtained in mice anesthetized with ketamin/xylazin, ${ }^{4}$ a volumetric flow rate of $0.54 \mathrm{ml} / \mathrm{min}(9 \mu \mathrm{l} / \mathrm{s})$ was used. Flow was always in physiological direction, i.e., the common carotid artery micropipette was connected to the linear pump, while the bifurcation micropipette was connected to the outflow tract. By increasing or decreasing the height of the outflow tract (Figure 5.1b), and, hence, the transmural pressure, the outer diameter was set at approximately $570 \mu \mathrm{m}$, resulting in an inner (lumen) diameter of about $500 \mu \mathrm{m}$ (carotid artery wall thickness is approximately $35 \mu \mathrm{m})^{17}$. As a consequence, the average flow velocity was approximately $45 \mathrm{~mm} / \mathrm{s}$, with corresponding centerline velocities of $90 \mathrm{~mm} / \mathrm{s}^{21}$

\section{Temporal resolution}

Temporal resolution was governed by the maximal repetition rate of the camera (32 frames per seconds; frame time: $31 \mathrm{~ms}$ ). To assess whether this resolution allowed adequate imaging of platelet-vessel wall interactions, the flow velocity near the vessel wall was determined from the length of platelet streaks using short exposure times ( $5 \mathrm{~ms}$ ). It was found to be approximately $1.5-4 \mathrm{~mm} / \mathrm{s}$ (not shown), indicating that platelets traveled between 45 and $125 \mu \mathrm{m}$ per frame, which fits well into the recording area width of $250 \mu \mathrm{m}$.

To assess flow pattern, longer exposure times $(20 \mathrm{~ms})$ were used, as this resulted in longer platelet streaks, giving a good indication of the (axially oriented) flow lines (Figure 5.2).

\section{Data acquisition}

Arteries were flushed with $1.5 \mathrm{ml}$ preheated buffer (either HBSS or BSA-SS; 35 - 37 ${ }^{\circ} \mathrm{C}$ ) containing a median of $1.6 \times 10^{7}$ platelets $/ \mathrm{ml}$ (interquartile range: $0.3-3.3$; normal concentration in mouse blood: approximately $1 \times 10^{9}$ platelets $\left./ \mathrm{ml}\right)$. Platelets and arteries were always from the same animal. Fluorescence microscopy was performed in the bifurcation just upstream of the flow divider region and in the 


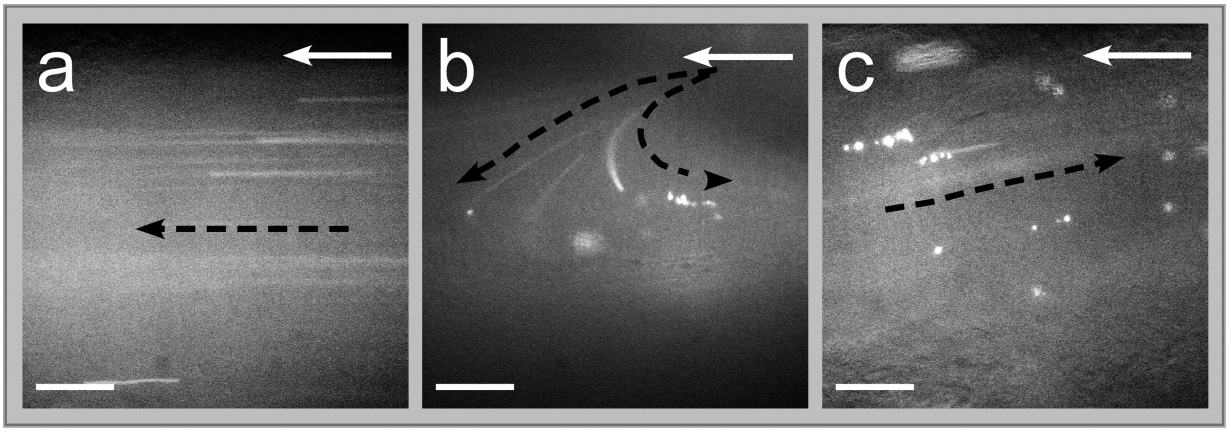

Figure 5.2 Developing flow pattern and platelet-vessel wall interactions.

Examples of wide-field fluorescence microscopic images of various local flow patterns in common carotid arteries. White arrow general flow direction (from common carotid artery (proximal) to carotid artery bifurcation (distal)); black dashed arrow observed local flow lines.

$a$ Situation where flow has developed into an axial pattern without any platelet-vessel wall interactions.

$b$ In the proximal common carotid artery, the flow profile has not yet fully developed. Separation of flow lines is visible (dashed arrows), creating flow reversal in the right part of the image with platelet string formation.

$c$ Area proximal to flow separation in $b$ resulting in flow reversal attended by platelet-vessel wall interactions (both adhesion and string formation).

Bars indicate $50 \mu \mathrm{m}$. Note that the area of recording does not cover the entire vessel diameter.

common carotid artery (Figure 5.1a). This way, recordings of 2 min were obtained per site for offline analysis.

Eight vessels were treated with hyaluronidase (type IV-S, $200 \mathrm{U} / \mathrm{ml}, 1 \mathrm{ml} / \mathrm{h}$ for 30 min; Sigma Aldrich, Zwijndrecht, The Netherlands); this enzymatic challenge is known to significantly reduce endothelial glycocalyx thickness in mounted carotid arteries. ${ }^{16}$ Immediately thereafter, the flushing/imaging sequence was carried out a second time at both vascular sites if possible (see below). In three vessels this enzymatic challenge was repeated.

Three vessels could not be used as the mounting procedure was compromised due to unavoidable leakage at the bifurcation. In three other instances, a low platelet count precluded imaging in both carotid arteries, as the first artery was treated with hyaluronidase, requiring a flushing/imaging sequence pre- and post-challenge, after which the platelet stock was depleted. For the same reason, imaging could not always be performed at both vascular sites. As a result, the final number of adequate recordings ( $\mathrm{B} 6 / \mathrm{ApoE}^{-/}$) was $15(11 / 4)$ in the common carotid artery and $13(8 / 5)$ in the bifurcation from a total of 16 vessels. 


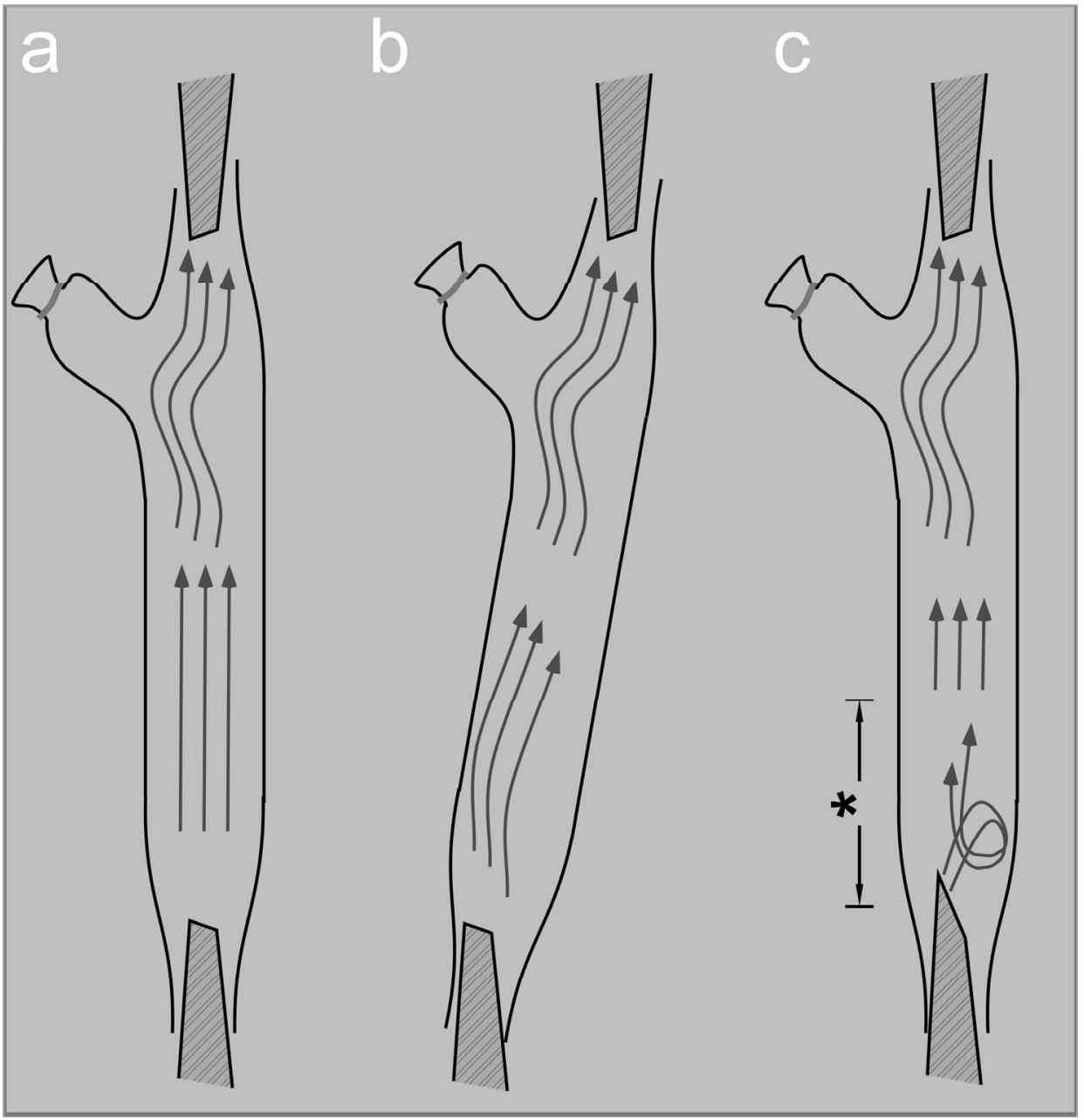

Figure 5.3 Flow patterns.

Schematic representation of flow patterns through mounted carotid arteries including the bifurcation.

$a$ Ideal situation in which the common carotid artery (proximal part) shows flow lines parallel to the longitudinal axis of the vessel (axial flow). In practice, the flow profile needs to develop over a certain part of the proximal common carotid artery (entrance length).

$b$ Situation in which the micropipettes are not optimally aligned, leading to an increased entrance length.

c Situation when the tip of the proximal micropipette is beveled too sharply, leading to flow separation in the common carotid artery, thus hampering normal development of the flow profile. The asterisk labels the entrance length. 


\section{Flow pattern entrance length}

Flow patterns developed into a (flattened) parabola ${ }^{22}$ over a certain length of the mounted common carotid artery (Figure 5.3). This entrance length could be estimated from overview recordings in most arteries (11 out of 16) and was expressed as length of the arterial segment subjected to undeveloped flow (asterisk in Figure 5.3c) divided by the lumen diameter. It was observed that the entrance length was (partly) governed by properties of the vessel chamber micropipettes, which could have been aligned poorly, or had a relatively sharp tip (Figure 5.3). In an effort to control entrance lengths, micropipettes were better aligned and their tips were blunted. Still, local geometry of the mounted vessel and pipette (tip) properties influenced the entrance length (in an unpredictable manner), resulting in variable entrance lengths between experiments. As undeveloped flow patterns were attended by extensive platelet-vessel wall interactions, recording was performed in parts of the vessel with axial flow lines (i.e. distal from the entrance length; Figure 5.2).

\section{Data analysis}

The recordings $(2 \mathrm{~min}$ ) were analyzed offline using the Wasabi software package (version 1.5, Hamamatsu Photonics). Two main categories of platelet-vessel wall interactions were identified: adhesion of single platelets and attachment of platelet strings or aggregates. As short-lasting single platelet interactions (saltating platelets) were scarce, only long-lasting single platelet interactions (platelet adhesion; $>20 \mathrm{~s}$ at the same location) were quantified and expressed as $\mathrm{n} / \mathrm{mm}^{2}$ per recording. In case there were more than 160 adhering platelets $/ \mathrm{mm}^{2}$ in one recording, this was classified as an adhesion score of $>160$ platelets $/ \mathrm{mm}^{2}$. Platelet aggregation and string formation was assessed in a nominal scale (0: none; 1 : single-sited; 2 : multisited). A platelet string was defined as 3 or more nonflowing platelets connected linearly by a string (invisible) in the direction of flow. ${ }^{27}$

\section{Statistical analysis}

Statistical analyses were performed using the SPSS software package (version 15.0, SPSS Inc., Chicago, USA). Data are presented as medians with interquartile ranges. The nonparametric Mann-Whitney $U$ test was used for comparison of groups. For comparison of coupled data, the nonparametric Wilcoxon's rank test was used. Spearman's $\rho$ was calculated as a measure for correlation. Statistical significance was accepted at $\alpha=0.05$. No coupled analysis was performed to respect the coupling of arteries originating from the same animal as variability between vessels 


\section{COMMON}

a
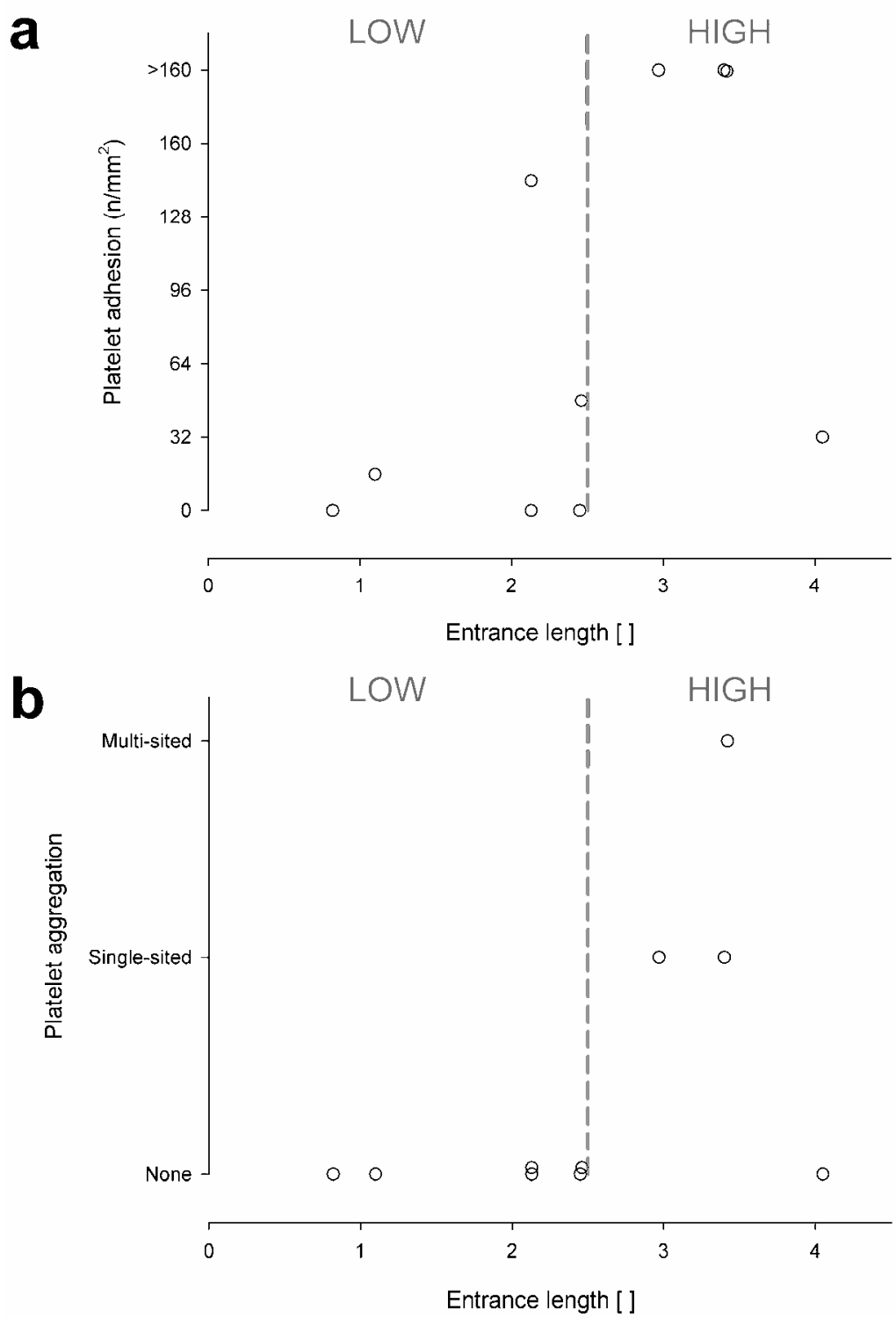

Figure 5.4 Influence of entrance length on platelet-vessel wall interactions.

a Number of adhering platelets to the vessel wall in the common carotid artery as a function of the entrance length. Note the increasing levels of platelet adhesion with increasing entrance lengths.

$b$ Occurrence of platelet aggregation at one (single-sited) or more (multi-sited) locations of the vessel wall in the common carotid artery as a function of the entrance length.

Gray dotted line indicates the cut-off point used to identify two separate platelet pre-activation groups based on the entrance length (Low and High, respectively). 
from the same animals was comparable to that of vessels from different animals (not shown).

\section{Results}

No differences in platelet-vessel wall interactions were found when stratifying groups for strain, age, used buffer (with or without albumin), or exact concentration of the platelet solution. Therefore, data were pooled.

\section{Influence of entrance length}

Strikingly, the entrance length affected platelet behavior downstream in the common carotid artery where flow was axial. As shown in Figure 5.4, a significant positive correlation was found between the entrance length and the number of platelet adhesions in the downstream common carotid artery (Spearman's $\rho=0.665, p<$ $0.05, \mathrm{n}=10$; Figure 5.4a). Furthermore, long entrance lengths were accompanied by the downstream attachment of platelet strings and aggregates (Figure 5.4b). In contrast, platelet adhesion was low in vessels with short entrance lengths, and no aggregation was seen. Apparently, the development of the flow profile led to a certain degree of platelet activation prior to interaction with the vessel wall downstream (platelet pre-activation). Therefore, vessels were divided into two platelet

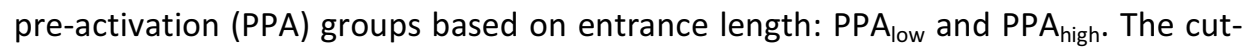
off for entrance length was set at 2.50 , resulting in two distinct groups of plateletvessel wall interactions (Figure 5.4, dashed line).

In 5 common carotid arteries (and 4 corresponding bifurcations), entrance lengths were not determined. One of these vessels showed aggregation and high platelet adhesion, and was therefore added to the PPA $A_{\text {high }}$ group. The other four vessels

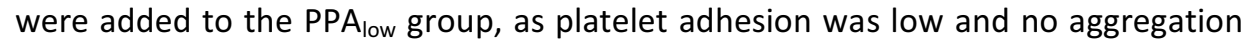
occurred. This way, the data fitted well into the groups with known entrance lengths; no essential statistical differences were induced (not shown).

As shown in Figure 5.5 (control data), the median level of platelet adhesion in the $\mathrm{PPA}_{\text {low }}$ group was found to be relatively low and not different between the common carotid artery and bifurcation (median (interquartile range): $8(0-36)$ and 0 (0 - 32) platelets $/ \mathrm{mm}^{2} ; \mathrm{n}=10$ and $\mathrm{n}=9$, respectively). In the PPA $A_{\text {high }}$ group, median numbers were $>160$ in both the common carotid artery $(64->160 ; n=5 ; p<0.01$ vs. $\left.\mathrm{PPA}_{\text {low }}\right)$ and the bifurcation $\left(44->160 ; n=4 ; p=0.06\right.$ vs. $\mathrm{PPA}_{\text {low }}$ ). 


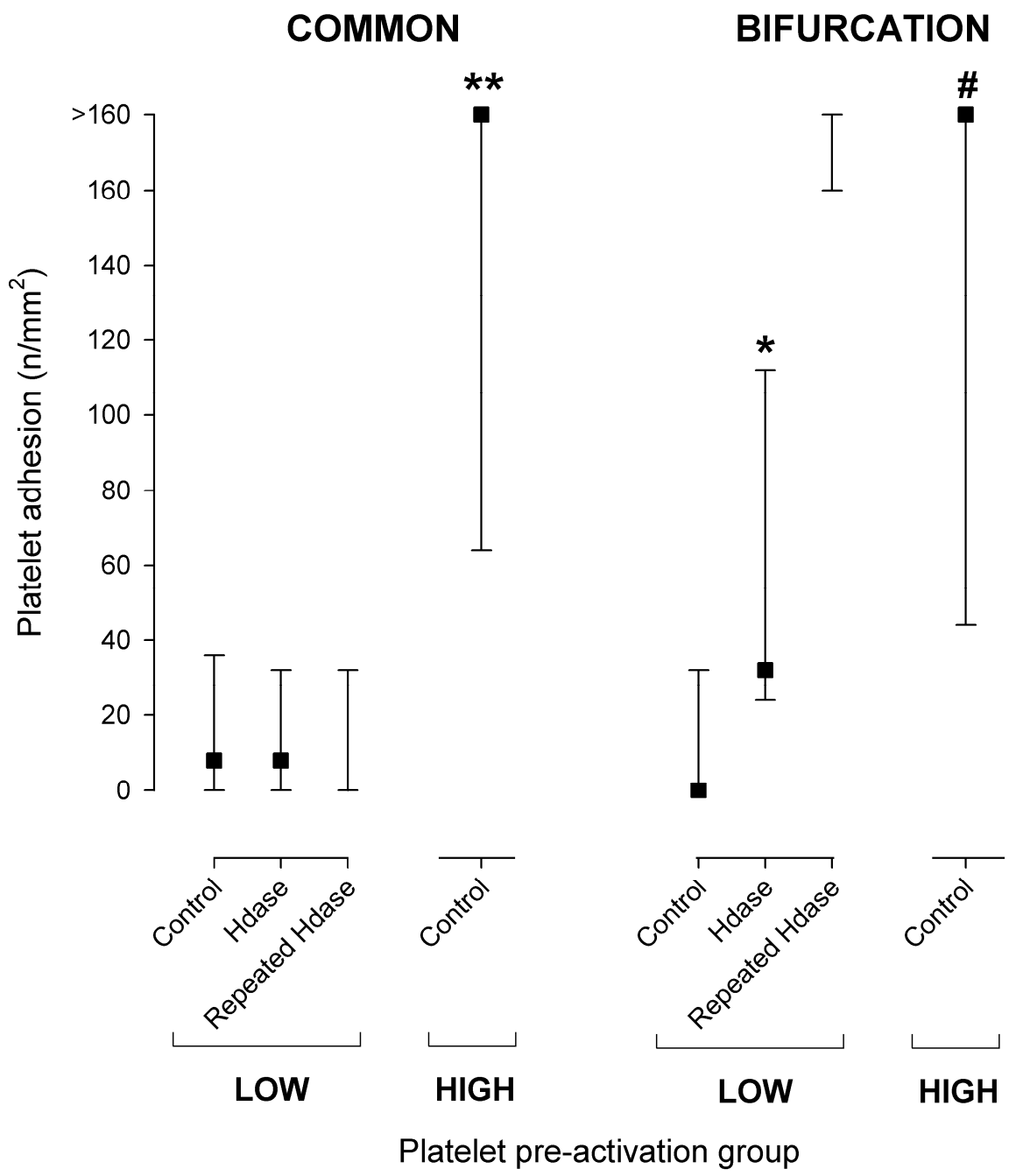

Figure 5.5 Platelet adhesion and the endothelial glycocalyx.

Platelet adhesion numbers in the common carotid artery (left) and carotid artery bifurcation (right). Vessels are divided into two platelet pre-activation groups based on entrance length (Low and High, respectively). Vessels from the Low group were enzymatically challenged with hyaluronidase (Hdase) to break down the endothelial glycocalyx. In some vessels this treatment was repeated. Data are presented as median \pm interquartile range. For the repeated Hdase groups the actual data range is given because of low $\mathrm{n}$ (common: 3 ; bifurcation: 2 ). ${ }^{*} \mathrm{p}<0.05, * * \mathrm{p}<0.01$, and $\# \mathrm{p}=0.06$ vs. Low control group. 


\section{Effect of removal of the endothelial glycocalyx}

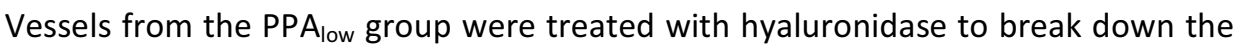
endothelial glycocalyx. In the bifurcation, platelet adhesion significantly increased to $32(24-112)$ platelets $/ \mathrm{mm}^{2}(p<0.05 ; n=5)$, while no change was observed in the common carotid artery $(8(0-32) ; n=6$; Figure 5.5$)$. Repeating the hyaluronidase challenge confirmed this finding. Again, no effect on platelet adhesion was observed in the common carotid artery (range $0-32 ; n=3$ ), while this additional breakdown of endothelial glycocalyx increased platelet adhesion in the bifurcation even more (range $160->160 ; n=2$; Figure 5.5).

\section{Discussion}

The present study indicates that it is possible to assess platelet-vessel wall interactions in isolated mouse carotid arteries at adequate temporal and spatial resolution. The level of platelet-vessel wall interactions appeared to be largely influenced by the flow pattern entrance lengths. In vessels with a short entrance length only low numbers of adhering platelets were observed in both the common carotid artery and the bifurcation. In these vessels, breakdown of the endothelial glycocalyx induced an increase in platelet adhesion in the carotid bifurcation only. This indicates that the shielding quality of the endothelial glycocalyx is relatively poor at this lesion-prone vascular site.

\section{Platelet pre-activation}

In the proximal common carotid artery, in areas where the undeveloped flow pattern had a direction that was nonaxial (Figures $5.2 b$ and $5.2 c$ ), many platelet-vessel wall interactions occurred. Furthermore, the longer this entrance length, the more platelet-vessel wall interactions were observed downstream. Probably, platelets are propelled into the glycocalyx at sites with nonaxial flow, facilitating their binding to receptors that cannot so easily be reached in case of axial flow. Once platelets bind to the endothelium, they can become activated, leading to attraction of other platelets (aggregation) and activation of endothelial cells, which in turn provides a positive feedback loop for platelet adhesion. Otherwise, in the absence of further stimulation, adhering platelets detach from the vessel wall and return to the circulation. $^{28}$

It is unlikely that platelets were pre-activated by shear effects, as the developing flow profile was still laminar. The estimated Reynolds number at a lumen diameter of $500 \mu \mathrm{m}$ and a volumetric flow rate of $45 \mathrm{~mm} / \mathrm{s}$ is approximately 34, when - for the sake of simplicity - assuming that the platelet buffer has the viscosity and den- 
sity of water at $40{ }^{\circ} \mathrm{C}\left(0.6531 \mathrm{mPa} \cdot \mathrm{s}\right.$ and $992.2 \mathrm{~kg} / \mathrm{m}^{3}$, respectively). ${ }^{21}$ This number is far too low for turbulence, and as such the effect of shear on platelet activation state can be neglected. From a Reynolds number of 34 follows a theoretical entrance length of $2.23 .^{21}$ Thus, the currently used cut-off value of 2.50 (Figure 5.4 ) is in line with theoretical models.

The entrance length-induced platelet pre-activation in our current setup may be used as a model for (patho)physiological platelet pre-activation in vivo such as occurs under conditions of hyperlipidemia and inflammation. ${ }^{28}$

In vessels with long entrance lengths, platelet adhesion and aggregation at sites with an axial flow pattern were significantly increased as compared to vessels with short entrance lengths (Figures 5.4 and 5.5). The exact mechanism through which platelet pre-activation leads to increased platelet-vessel wall interactions at downstream sites exposed to axial flow remains to be elucidated. We hypothesize that activated platelets by themselves are better able to reach the endothelium, e.g., by tether formation (platelet-specific effect), ${ }^{4}$ and that in the area of the entrance length various molecules are released from the pre-activated platelets and/or endothelium into the perfusate that may activate the endothelium and/or passing platelets downstream (model-generic effect). Both effects would increase the likelihood of platelet-vessel wall interactions downstream.

\section{Comparability to in vivo data - role of the glycocalyx}

In vessels with a short entrance length, the low number of adhering platelets (8 platelets $/ \mathrm{mm}^{2}(0-36)$ in the common carotid artery and $0(0-32)$ in the bifurcation) was comparable to the in vivo situation of wild-type controls $(0(0-16)$ for both locations). ${ }^{4}$ This is consistent with previous reports that freshly excised and mounted carotid arteries have an intact, viable vessel wall endowed with an endothelial glycocalyx of about $2-3 \mu \mathrm{m}$ thick. ${ }^{4,16,17}$ Breakdown of the glycocalyx in these vessels through hyaluronidase perfusion consistently increased platelet adhesion in the bifurcation. Interestingly, no changes were observed in the common carotid artery. This indicates a spatial difference in shielding capacity of the endothelial glycocalyx, which might be (partly) explained by a difference in the local flow profile. In the common carotid artery, which is exposed to axial flow, a reduction in glycocalyx thickness did not increase platelet adhesion. In the bifurcation, however, where flow is not fully axial, ${ }^{29}$ glycocalyx breakdown resulted in an increased likelihood of platelet adhesion. As such, the importance of the glycocalyx in modulating platelet-vessel wall interactions seems larger in the bifurcation. This also corre- 
sponds to our in vivo data, showing a reduced glycocalyx thickness in the bifurcation of atherogenic mice, coinciding with increased levels of platelet adhesion. ${ }^{4}$

In the current study, no strain difference in the control level of platelet adhesion in the bifurcation was found, which might be attributed to a low group size induced by entrance length variability (e.g., $\mathrm{ApoE}^{-/-}$bifurcations in the PPA $\mathrm{low}_{\text {ow }}$ group: $n=3$ ). The same applies to the influence of age on the number of platelet adhesions.

\section{Model benefits and limitations}

The presented model has several advantages over in vivo assessment of plateletvessel wall interactions: (1) It offers the opportunity to perform stable imaging of platelet adhesion and aggregation at the level of the arterial wall without vessel movement and at adequate spatial and temporal resolution. (2) Extended experiments can be performed without the limitation of fluid overload such as occurs rather easily during in vivo experiments on mice. From previous studies it is known that isolated carotid arteries remain viable for at least $4 \mathrm{~h}^{17}{ }^{17}$ (3) Experiments are comparable with respect to shear rate in those parts where flow patterns are (close to) fully developed; when assuming an equal viscosity of the platelet buffer at the current platelet concentrations, shear stresses are comparable as well. (4) Many possibilities for modulating vessel wall and/or platelet properties to study their role in platelet behavior in large arteries are offered (some of which are also available in the in vivo situation). Examples of the latter are the use of enzymes to modify the vessel wall, agents and/or dyes to trigger and study endothelial cell activation, specific antibodies against endothelial cell or platelet ligands/receptors, vessels and/or platelets from genetically modified mouse strains, and platelet populations from a certain genetic background in the vessel of another. Furthermore, platelet preactivation by long entrance lengths can be used to deliberately induce plateletvessel wall interactions.

Unfortunately, the current approach also comes with several disadvantages: (1) Despite our effort to minimize the entrance length of the developing flow pattern, it can only be controlled partially, leading to loss of data and experimental resources. (2) The choice for motionless imaging by inducing continuous flow means a simplification of the in vivo situation where flow is pulsatile. Pulsatile flow can be mimicked in our model, but would lead to motion artifacts, out-of-focus frames and thereby to a reduction of the temporal resolution. (3) Both platelets and arteries are isolated, and one side branch of the carotid artery bifurcation is ligated during the mounting process. This will influence platelet and vessel wall behavior, as 
well as local flow profiles. Therefore, validation of any data acquired using this model with data from corresponding in vivo experiments is needed. (4) The model does not take into account the rheological role of red blood cells, that influences platelet distribution in vivo and, hence, local platelet concentration. ${ }^{22}$ Adding red blood cells to the solution is a possibility, but this will most likely affect the yield of fluorescence, as they are known to absorb excitation light, ${ }^{30}$ whereas the use of platelet solutions provides excellent penetration of light and, hence, a good signalto-noise ratio. (5) At the current diameter and flow rate settings, very short exposure times are needed to study individual platelet behavior in detail, leading to a deterioration of the signal-to-noise ratio. The temporal resolution is mainly governed by the repetition rate of the camera. Decreasing the flow rate improves the balance between temporal resolution, signal-to-noise ratio and flow velocity, but at the cost of comparability to the in vivo situation. Alternatively, a larger area of recording can be achieved by using a different objective, but at the cost of spatial resolution. In practice, the exact research issue at hand will determine the needed area of recording, flow velocity, exposure time and magnification. (6) In the current setup, the amount of platelet stock is a limiting factor for the number of recordings per vessel. Several solutions can be applied, such as pooling platelets from multiple animals to enable more recordings per vessel (e.g., after various interventions). In this study, no effect of increasing platelet concentration (range $1.4-51.5 \times 10^{7}$ platelets $/ \mathrm{ml}$ ) on the number of platelet adhesions was observed, suggesting that the amount of flushed platelets is not a limiting factor for the number of plateletvessel wall interactions. As an alternative setup, inactive fluorescent particles could be used to first image and optimize the flow pattern and entrance length, at the same time avoiding any vessel or platelet activation during this process.

\section{Conclusions}

We present a straightforward model to study platelet behavior in isolated mouse carotid arteries at adequate optical and temporal resolution. The entrance length of the developing flow pattern was found to be a major determinant of plateletvessel wall interactions. Taking this into account, a protective role for the endothelial glycocalyx against platelet adhesion at the carotid bifurcation was found. This model can be easily adapted to study a variety of platelet- or vessel wall-dependent factors in platelet-vessel wall interactions.

\section{Acknowledgements}

This research is supported by NWO grant \#902-16-276 and SenterNovem (BSIK 03033). 


\section{References}

1. Leslie M. Cell biology. Beyond clotting: the powers of platelets. Science. 2010;328:562-564.

2. Libby P. Inflammation in atherosclerosis. Nature. 2002;420:868-874.

3. Hansson GK. Inflammation, atherosclerosis, and coronary artery disease. $N$ Engl J Med. 2005;352:1685-1695.

4. Reitsma S, oude Egbrink MGA, Heijnen VVT, Megens RT, Engels W, Vink H, Slaaf DW, van Zandvoort MAMJ. Endothelial glycocalyx thickness and platelet-vessel wall interactions during atherogenesis. Thromb Haemost. 2011;106:939-946.

5. Huo Y, Schober A, Forlow SB, Smith DF, Hyman MC, Jung S, Littman DR, Weber C, Ley K. Circulating activated platelets exacerbate atherosclerosis in mice deficient in apolipoprotein E. Nat Med. 2003;9:61-67.

6. Weber C. Platelets and chemokines in atherosclerosis: partners in crime. Circ Res. 2005;96:612616.

7. Massberg S, Brand K, Gruner S, Page S, Muller E, Muller I, Bergmeier W, Richter T, Lorenz M, Konrad I, Nieswandt B, Gawaz M. A critical role of platelet adhesion in the initiation of atherosclerotic lesion formation. J Exp Med. 2002;196:887-896.

8. Schulz C, Konrad I, Sauer S, Orschiedt L, Koellnberger M, Lorenz R, Walter U, Massberg S. Effect of chronic treatment with acetylsalicylic acid and clopidogrel on atheroprogression and atherothrombosis in ApoE-deficient mice in vivo. Thromb Haemost. 2008;99:190-195.

9. Vink $\mathrm{H}$, Constantinescu AA, Spaan JA. Oxidized lipoproteins degrade the endothelial surface layer: implications for platelet-endothelial cell adhesion. Circulation. 2000;101:1500-1502.

10. van Gestel MA, Heemskerk JW, Slaaf DW, Heijnen VV, Sage SO, Reneman RS, oude Egbrink MG. Real-time detection of activation patterns in individual platelets during thromboembolism in vivo: differences between thrombus growth and embolus formation. J Vasc Res. 2002;39:534-543.

11. Megens RT, Reitsma S, Prinzen L, oude Egbrink MG, Engels W, Leenders PJ, Brunenberg EJ, Reesink $K D$, Janssen BJ, ter Haar Romeny BM, Slaaf DW, van Zandvoort MA. In vivo high-resolution structural imaging of large arteries in small rodents using two-photon laser scanning microscopy. $J$ Biomed Opt. 2010;15:011108.

12. Kuijpers MJ, Schulte V, Bergmeier W, Lindhout T, Brakebusch C, Offermanns S, Fassler R, Heemskerk JW, Nieswandt B. Complementary roles of glycoprotein VI and alpha2beta1 integrin in collagen-induced thrombus formation in flowing whole blood ex vivo. Faseb J. 2003;17:685-687.

13. Hafezi-Moghadam A, Thomas KL, Cornelssen C. A novel mouse-driven ex vivo flow chamber for the study of leukocyte and platelet function. Am J Physiol Cell Physiol. 2004;286:C876-892.

14. Reitsma S, Slaaf DW, Vink H, van Zandvoort MA, Oude Egbrink MG. The endothelial glycocalyx: composition, functions, and visualization. Pflugers Arch. 2007;454:345-359.

15. Potter DR, Damiano ER. The hydrodynamically relevant endothelial cell glycocalyx observed in vivo is absent in vitro. Circ Res. 2008;102:770-776.

16. Reitsma S, oude Egbrink MGA, Vink H, van den Berg B, Lima Passos V, Engels W, Slaaf DW, van Zandvoort MAMJ. Endothelial glycocalyx structure in the intact carotid artery: a two-photon laser scanning microscopy study. J Vasc Res. 2011;48:297-306.

17. Megens RT, Reitsma S, Schiffers PH, Hilgers RH, De Mey JG, Slaaf DW, oude Egbrink MG, van Zandvoort MA. Two-photon microscopy of vital murine elastic and muscular arteries. Combined structural and functional imaging with subcellular resolution. J Vasc Res. 2007;44:87-98.

18. Bergaya S, Meneton P, Bloch-Faure M, Mathieu E, Alhenc-Gelas F, Levy BI, Boulanger CM. Decreased flow-dependent dilation in carotid arteries of tissue kallikrein-knockout mice. Circ Res. 2001;88:593-599. 
19. Ramos CL, Huo Y, Jung U, Ghosh S, Manka DR, Sarembock IJ, Ley K. Direct demonstration of Pselectin- and VCAM-1-dependent mononuclear cell rolling in early atherosclerotic lesions of apolipoprotein E-deficient mice. Circ Res. 1999;84:1237-1244.

20. Caro CG, Nerem RM. Transport of 14 C-4-cholesterol between serum and wall in the perfused dog common carotid artery. Circ Res. 1973;32:187-205.

21. Wood NB. Aspects of fluid dynamics applied to the larger arteries. J Theor Biol. 1999;199:137-161.

22. Tangelder GJ, Slaaf DW, Muijtjens AM, Arts T, oude Egbrink MG, Reneman RS. Velocity profiles of blood platelets and red blood cells flowing in arterioles of the rabbit mesentery. Circ Res. 1986;59:505-514.

23. Cheng C, van Haperen R, de Waard M, van Damme LC, Tempel D, Hanemaaijer L, van Cappellen GW, Bos J, Slager CJ, Duncker DJ, van der Steen AF, de Crom R, Krams R. Shear stress affects the intracellular distribution of eNOS: direct demonstration by a novel in vivo technique. Blood. 2005;106:3691-3698.

24. van Bochove GS, Straathof R, Krams R, Nicolay K, Strijkers GJ. MRI-determined carotid artery flow velocities and wall shear stress in a mouse model of vulnerable and stable atherosclerotic plaque. Magma. 2010;23:77-84.

25. Rudic RD, Bucci M, Fulton D, Segal SS, Sessa WC. Temporal events underlying arterial remodeling after chronic flow reduction in mice: correlation of structural changes with a deficit in basal nitric oxide synthesis. Circ Res. 2000;86:1160-1166.

26. Janssen BJ, De Celle T, Debets JJ, Brouns AE, Callahan MF, Smith TL. Effects of anesthetics on systemic hemodynamics in mice. Am J Physiol Heart Circ Physiol. 2004;287:H1618-1624.

27. Dong JF, Moake JL, Nolasco L, Bernardo A, Arceneaux W, Shrimpton CN, Schade AJ, McIntire LV, Fujikawa K, Lopez JA. ADAMTS-13 rapidly cleaves newly secreted ultralarge von Willebrand factor multimers on the endothelial surface under flowing conditions. Blood. 2002;100:4033-4039.

28. Wagner DD, Frenette PS. The vessel wall and its interactions. Blood. 2008;111:5271-5281.

29. Ku DN, Giddens DP. Pulsatile flow in a model carotid bifurcation. Arteriosclerosis. 1983;3:31-39.

30. Kaestner L, Tabellion W, Weiss E, Bernhardt I, Lipp P. Calcium imaging of individual erythrocytes: problems and approaches. Cell Calcium. 2006;39:13-19. 



\section{In vivo high-resolution structural} imaging of large arteries in small rodents using two-photon laser

\section{scanning microscopy}

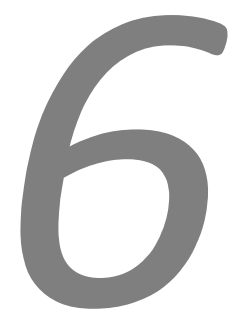

Published as

Remco T.A. Megens*, Sietze Reitsma*, Lenneke Prinzen, Mirjam G.A. oude Egbrink, Wim Engels, Peter J.A.Leenders, Ellen J. L. Brunenberg, Koen D. Reesink, Ben J.A. Janssen, Bart M. ter Haar Romeny, Dick W. Slaaf, Marc A.M.J. van Zandvoort

Journal of Biomedical Optics, 2010; 15:011108

Including online additional videos:

http://spiedigitallibrary.org/jbo/resource/1/jbopfo/v15/i1/p011108_s1

* both authors contributed equally 


\begin{abstract}
In vivo (molecular) imaging of the vessel wall of large arteries at subcellular resolution is crucial for unraveling vascular pathophysiology. We previously showed the applicability of two-photon laser scanning microscopy (TPLSM) in mounted arteries ex vivo. However, in vivo TPLSM has thus far suffered from in-frame and betweenframe motion artifacts due to arterial movement with cardiac and respiratory activity. Now, motion artifacts were suppressed by accelerated image acquisition triggered on cardiac and respiratory activity. In vivo TPLSM is performed on rat renal and mouse carotid arteries, both surgically exposed and labeled fluorescently (cell nuclei, elastin, and collagen). The use of short acquisition times consistently limits in-frame motion artifacts. Additionally, triggered imaging reduces between-frame artifacts. Indeed, structures in the vessel wall (cell nuclei, elastic laminae) could be imaged at subcellular resolution. In mechanically damaged carotid arteries, even the subendothelial collagen sheet (about $1 \mu \mathrm{m}$ ) was visualized using collagentargeted quantum dots. We demonstrate stable in vivo imaging of large arteries at subcellular resolution using TPLSM triggered on cardiac and respiratory cycles. This creates great opportunities for studying of (diseased) arteries in vivo or immediate validation of in vivo molecular imaging techniques such as magnetic resonance imaging (MRI), ultrasound, and positron emission tomography (PET).
\end{abstract}




\section{Introduction}

In the past decades, imaging of (patho)physiological processes in large (conduit) arteries has become increasingly important in the context of diseases such as atherosclerosis. Recently, we and others have put much effort in developing techniques to visualize vascular structure and function especially in large murine vessels. We recently published an ex vivo setup based on two-photon laser scanning microscopy (TPLSM), which enables structural and functional fluorescent imaging of intact, viable larger arteries of mice at a subcellular resolution. ${ }^{1}$ However, realtime in vivo imaging in these vessels at such a resolution has not been reported yet.

Conventional intravital brightfield and fluorescence microscopy techniques are particularly suitable for imaging small blood vessels in thin transparent tissues where detection of fluorescent blood cells is possible up to a depth of $100 \mu \mathrm{m}$. They enable among other advantages, visualization of blood cell-vessel wall interactions $^{2,3}$ and hemodynamic parameters. ${ }^{4}$ It was demonstrated that conventional intravital fluorescence microscopy also allows for detection of fluorescently labeled blood cells that interact with the vessel wall in large (atherosclerotic) arteries in mice (diameter up to $600 \mu \mathrm{m}$ ). ${ }^{5-8}$ However, this technique is not suitable for imaging (subcellular) structures in the vessel wall of these large arteries. Image quality is strongly hampered by contributions from out-of-focus and scattered fluorescence, and rapidly decreases at deeper layers. This precludes the use of conventional intravital fluorescence microscopy for in vivo visualization of subcellular structures in the vessel wall of larger arteries.

Confocal laser scanning microscopy offers excellent high-resolution images of vascular structures in vivo., 10 However, spatial resolution gradually deteriorates deeper in the sample $(>40 \mu \mathrm{m})$ due to out-of-focus scattered fluorescence light passing through the pinhole. Furthermore, the focal-to-nonfocal signal ratio decreases rapidly with depth, leading to poor penetration depth. ${ }^{11}$ In contrast, the spatial resolution of TPLSM is less affected by scattering. Two-photon excitation of fluorescent molecules uses a wavelength that is twice that of single photon excitation. This higher excitation wavelength is less susceptible to scattering. Furthermore, two-photon excitation occurs only in a small volume element (voxel) in the focal plane. Any light reaching the objective lens originates from the excited voxel, independent of the amount of scattering experienced by the emitted light. This results in preservation of spatial resolution in scattering tissues and optical sectioning. The combination of increased penetration depth, good optical sectioning, and subcellular resolution enables ex vivo TPLSM imaging of large arteries at high spa- 
tial resolution. ${ }^{1,11-15}$ Also in vivo TPLSM has been reported in various relatively motionless tissues and structures, such as brain, ${ }^{16}$ kidney, ${ }^{17-21}$ microvasculature, ${ }^{22}$ skin, $^{23,24}$ and lymphatics. ${ }^{25}$

The complication of imaging of large arteries in vivo originates from cardiac and respiratory activity, which results in a repetitive but variable pattern of vessel movement. Random acquisition of images within this pattern will result in images that reflect different parts of vessel motion (between-frame motion artifacts). In this way, such images contain random parts of the vessel and are not mutually comparable. Furthermore, part of the vessel movement is captured within each image, resulting in image distortion (in-frame motion artifacts). In conventional fluorescence microscopy, image distortion may be eliminated by using flashed illumination at the right period during the cardiac and respiratory cycles. Unfortunately, TPLSM does not allow instantaneous image acquisition, since image composition is based on point-by-point scanning through the optical section. The diameter of large arteries in small rodents is about $400-600 \mu \mathrm{m}$, and their distention is significantly larger than the thickness of the optical section, which is only about 1.5 $\mu \mathrm{m}$ in TPLSM. This induces image distortion and challenges its use in imaging of large arteries in vivo. ${ }^{11}$ Some researchers have evaded this problem by temporarily stopping blood flow. ${ }^{26}$ This approach, however, severely disrupts the physiological conditions in these vessels. Moreover, it cannot be used to visualize dynamic processes such as blood cell-vessel wall interactions. In other imaging modalities, such as magnetic resonance imaging, triggered image acquisition is used to overcome these motion artifacts.

In this study, we propose and test whether for applications of TPLSM in vivo, motion artifacts can be minimized by making several adaptations to the acquisition mode. One is whether acceleration of image acquisition will decrease image distortion. This can be achieved by reducing the number of pixels or the pixel dwell-times (or a combination of both). A drawback of accelerated image acquisition is a reduction of signal-to-noise ratio (SNR). Second, the acquisition of each separate image in a time sequence should start at a specific, fixed moment of the cardiac and respiration cycle. This potentially results in a series of images that all contain a similar part of the moving arterial wall, thus limiting between-frame motion artifacts. Finally, the application of specific image processing tools may improve overall image quality.

Imaging was performed in surgically exposed arteries of anesthetized mice and rats, which were stained with specific fluorescent markers. As a proof of principle 
for the method and its applicability for testing molecular imaging agents in vivo, damage-induced exposure of the thin subendothelial collagen sheet was visualized using collagen-targeted quantum dots.

\section{Materials and Methods}

\section{Animals}

All experiments were in line with institutional guidelines and approved by the local ethics committee on the use of laboratory animals. Mice (C57BL6/J; $n=7$ ) were anesthetized using subcutaneous administration of a mixture of $75 \mathrm{mg} / \mathrm{kg}$ ketamin (Nimatek, Eurovet, Cuijck, the Netherlands) and $15 \mathrm{mg} / \mathrm{kg}$ xylazin (Xylazin, Ceva Sante Animale BV, Naaldwijk, the Netherlands) in a volume of $3 \mathrm{ml} / \mathrm{kg}$. Anesthesia was maintained by subcutaneous injection of $0.88 \mathrm{ml} / \mathrm{kg}$ of the same xylazinketamin mixture every 30 min. Rats (Wistar-Kyoto; $n=6$ ) were anesthetized by a single intraperitoneal injection of urethane $(1.7 \mathrm{mg} / \mathrm{g})$. All anesthetics were dissolved in saline. Body temperature was monitored using a rectal probe (PT100 sensor, Watlow, UK) and maintained at approximately $37^{\circ} \mathrm{C}$ by a heating platform (TH60-SMZ, Linkam Scientific Instruments, UK).

\section{Tissue preparation and instrumentation}

Animals were placed in supine position. In mice, the right common carotid artery was surgically exposed up to the bifurcation. ${ }^{5}$ In rats, the abdominal region was surgically opened to expose the left renal artery. To simplify discrimination between artery and surrounding tissues in both preparations, a thin black plastic sheet was placed underneath the artery segments without stretching the vessel. Exposed areas were kept moist with saline at all time during the experiment.

In both species, the left jugular vein was cannulated for administration of fluorescent dyes. Blood pressure was measured through a catheter inserted in the left femoral artery and linked to two blood pressure sensors (Baxter Uniflow, Baxter B.V., Utrecht, the Netherlands); the first sensor was used to monitor blood pressure and, hence, the hemodynamic condition of the animal. The second blood pressure signal was used as input for image triggering (see later in the paper). All catheters and blood pressure probes were filled with heparin $(5 \mathrm{U} / \mathrm{ml}$ in saline).

To stabilize respiration, the trachea was intubated and ventilated with normal air using a ventilator (mouse: Minivent 845, Hugo Sachs Electronic GmbH, Germany; rat: Harvard rodent respirator, Harvard Apparatus, Mass., USA). As heart rate and also vessel motion may vary slightly from changes in intrathoracic pressure due to breathing, a respiration sensor (Graseby, Wicklow, Ireland) was placed on top of 
the chest in order to obtain a (relative) respiration signal for the trigger unit. In a number of mice ( $n=3$ ), no (forced) ventilation was used to investigate the effect of spontaneous breathing on image motion and triggering.

\section{Fluorescent labeling}

Nuclei of viable cells were labeled by topical and/or intravenous administration of the viable cell membrane permeable DNA / RNA markers SYTO13 or SYTO41 (Molecular Probes, Leiden, the Netherlands). Eosin (Molecular Probes) was used as a specific fluorescent marker for elastin. SYTO13, SYTO41 (both $2.0 \mu \mathrm{M}$ ) and eosin $(0.5 \mu \mathrm{M})$ were dissolved in saline and administered topically on the artery. In four rats and three mice, SYTO13 was infused intravenously $(0.1 \mathrm{ml}$ of a $4.0 \mu \mathrm{M}$ solution in saline) to more specifically stain the endothelial cell nuclei and blood cells. Circulating blood platelets, cytoplasm of leukocytes, and cells in the arterial wall were labeled fluorescently by intravenous administration (i.v.) of acridin red (ChromaGesellschaft Schmidt GmbH, Germany; $2.0 \mathrm{mg} / \mathrm{ml}$ in saline with $5 \%$ ethanol; a bolus of $0.03 \mathrm{ml}$ in mice or $0.1 \mathrm{ml}$ in rats). Collagen (types I and III) in the tunica adventitia was visualized by second-harmonic generation (SHG). The subendothelial collagen layer (type IV) is too thin and located too deep to be detected by SHG on our system, especially in vivo. ${ }^{1,12}$ Therefore, subendothelial collagen, which is luminally exposed on damage to the endothelium, was visualized using collagen-specific CNA35 ${ }^{12,27}$ labeled with green fluorescent quantum dots (CNA35-QD525; $200 \mu$ li.v. containing $15 \mu \mathrm{M}$ CNA35-biotin and $0.5 \mu \mathrm{M}$ streptavidin coated quantum dots (Invitrogen)). Propidium iodide was used to label nonviable cells (PI; Invitrogen; $200 \mu \mathrm{l}$ of a $0.01 \mathrm{mg} / \mathrm{ml}$ solution applied topically).

\section{Damage-induced subendothelial collagen exposure}

In a subset of experiments, the carotid artery of mice was pinched with a finetipped forceps for $3 \mathrm{~s}$ to induce damage across the vessel wall. In our ex vivo setup, this procedure has been shown to be effective both in inducing cell damage across the vessel wall, ${ }^{1}$ and in uncovering the subendothelial collagen layer. ${ }^{12}$ Then, the CNA35-QD525 solution was infused i.v. to label exposed subendothelial collagen. ${ }^{27}$ Furthermore, propidium iodide was applied topically to label nonviable cells.

\section{Image acquisition}

A Nikon E600FN microscope (Nikon Corporation, Tokyo, Japan) connected to a Biorad 2100 MP multiphoton system (Biorad, Hemel Hempstead, UK) was used for TPLSM as previously described. ${ }^{1,28}$ 
Table 6.1 Image acquisition characteristics

\begin{tabular}{|c|c|c|c|c|c|}
\hline \multicolumn{3}{|c|}{$\begin{array}{l}\text { Image acquisition time (s) } \\
\text { Scanning mode }\end{array}$} & \multirow[t]{2}{*}{$\begin{array}{l}\text { Line scan rate } \\
\left.\text { (lines } \cdot \mathrm{s}^{-1}\right)\end{array}$} & \multirow[t]{2}{*}{ Optical zoom } & \multirow[t]{2}{*}{$\begin{array}{l}\text { Maximum FOV } \\
\left(\mu \mathrm{m}^{2}\right)\end{array}$} \\
\hline $1 x$ & $2 x$ & $4 x$ & & & \\
\hline 10.24 & 5.12 & 2.56 & 25 & 1.0 & $206 \times 206$ \\
\hline 5.12 & 2.56 & 1.28 & 50 & 1.0 & $206 \times 206$ \\
\hline 1.54 & 0.77 & 0.39 & 166 & 1.0 & $206 \times 206$ \\
\hline 0.51 & 0.26 & 0.13 & 500 & 1.0 & $206 \times 206$ \\
\hline 0.43 & 0.21 & 0.11 & 600 & 1.2 & $172 \times 172$ \\
\hline 0.34 & 0.17 & 0.09 & 750 & 1.5 & $137 \times 137$ \\
\hline $0.21^{\dagger}$ & $0.11^{\dagger}$ & $0.05^{\dagger}$ & 1200 & 1.0 & $206 \times 206$ \\
\hline $0.17^{\dagger}$ & $0.09^{\dagger}$ & $0.04^{+}$ & 1500 & 1.2 & $172 \times 172$ \\
\hline $0.14^{\dagger}$ & $0.07^{\dagger}$ & $0.04^{+}$ & 1800 & 1.6 & $126 \times 126$ \\
\hline
\end{tabular}

\footnotetext{
${ }^{\dagger}$ Introduction of (correctable) interlacing errors due to bidirectional scanning. Scanning modes indicate whether 1, 2, or 4 lines are scanned at the same time. Double and quadruple modes increase scanning speed, but decrease spatial resolution in vertical direction. FOV field of view; image acquisition times assume frames of $256 \times 256$ pixels
}

A $120 \pm 20$ fs-pulsed Ti:Sapphire laser (Spectra Physics Tsunami, Mountain View, Calif., USA) was used as the excitation source tuned and mode-locked at 800 to 840 $\mathrm{nm}$. Excitation powers at the sample varied from 60 to $80 \mathrm{~mW}$. Either a $40 \times$ or a $60 \times$ water dipping objective was used (numerarical aperture (NA) 0.8 and 1.0, respectively) for TPLSM recordings. A 20x water dipping objective (NA 0.5) was used in combination with a 20-W halogen cold light source (Schott KL 200, Schott AG, Mainz, Germany) to locate the exposed vessels. To detect the emitted fluorescent signals, three photomultiplier tubes were used. These were tuned to correspond with parts of the emission spectra of the fluorescent markers applied, aiming to find a balance between minimal bleed-through versus maximal signal reception. ${ }^{1}$ Images of $256 \times 256$ pixels were obtained for each color channel and combined into one RGB image. Series of 20 to 100 subsequent xy images were recorded (time series). Acquisition of each separate image was started by a trigger pulse derived from a trigger unit (see later in the paper). Optimal image acquisition time was determined empirically depending on heart rate (see later in the paper).

Table 6.1 summarizes possible image acquisition times and settings of the system. Image acquisition times applied in this paper varied from 0.11 to $0.43 \mathrm{~s}$, depending on matrix size, pixel dwell-time, line speed, and artery/species. When imaging at $>500$ lines/s, optical zoom is automatically applied in order to reduce total scan 
area, and hence, image acquisition time. As an advantage, spatial resolution is increased. Furthermore, line speeds of $>750$ lines/s are achieved through bidirectional scanning, which increases scanning speed (requiring no or less optical zoom), and also results in (correctable) interlacing errors (see later in the paper). Image acquisition time can also be decreased by using double and quadruple line scanning modes, where two or four lines are scanned at the same time. This results in a decrease of spatial resolution in vertical direction ( $y$ direction), which can be corrected by applying additional zoom at the cost of FOV. Objects of interest should therefore be aligned in such a way that the maximum spatial resolution is obtained in the required direction.

\section{Triggering}

The respiration sensor and one of the blood pressure sensors were connected to an electrocardiogram (ECG)-trigger unit (Rapid Biomedical, Würzburg, Germany), originally designed for application in magnetic resonance imaging. However, an ECG could not be used as input, since positioning of an objective lens on the animal strongly disturbed the recorded electrical activity. Therefore, the blood pressure signal derived from the femoral artery was fed into the ECG input, which resulted in modification of the signal. The output trigger signal was coupled to the trigger/synchronizer input port of the instrumentation control unit of the TPLSM system. The trigger unit displayed both respiration and modified blood pressure signals, and for each a threshold could be set (Figure 6.1). The blood pressure signal was used as the primary determinant for the trigger moment, aiming to generate one trigger pulse per cardiac cycle. Furthermore, the influence of respiration on motion artifacts was minimized using the respiratory signal. Whenever the respiratory signal exceeded its threshold (during inspiration), triggering was disabled. If, however, the respiration signal was below threshold and the modified blood pressure signal crossed its threshold while rising, a trigger pulse was generated, initiating the acquisition of a single optical section. A trigger delay for both blood pressure and respiration could also be set. This simplified the search for an optimal trigger moment (during diastole and expiration) without the need for adjustment of the actual blood pressure signal, respiration signal, or thresholds. Trigger pulse, modified blood pressure, and respiration signals from the trigger unit, and absolute blood pressure signal were digitally recorded using an acquisition system M-PAQ in combination with the acquisition software package IDEEQ (IDEE, Maastricht, the Netherlands). Sampling rate was $1000 \mathrm{~Hz}$. Figure 6.1 shows such a recording for the duration of $6 \mathrm{~s}$. Total frame time, start time of each subsequent image, and time 


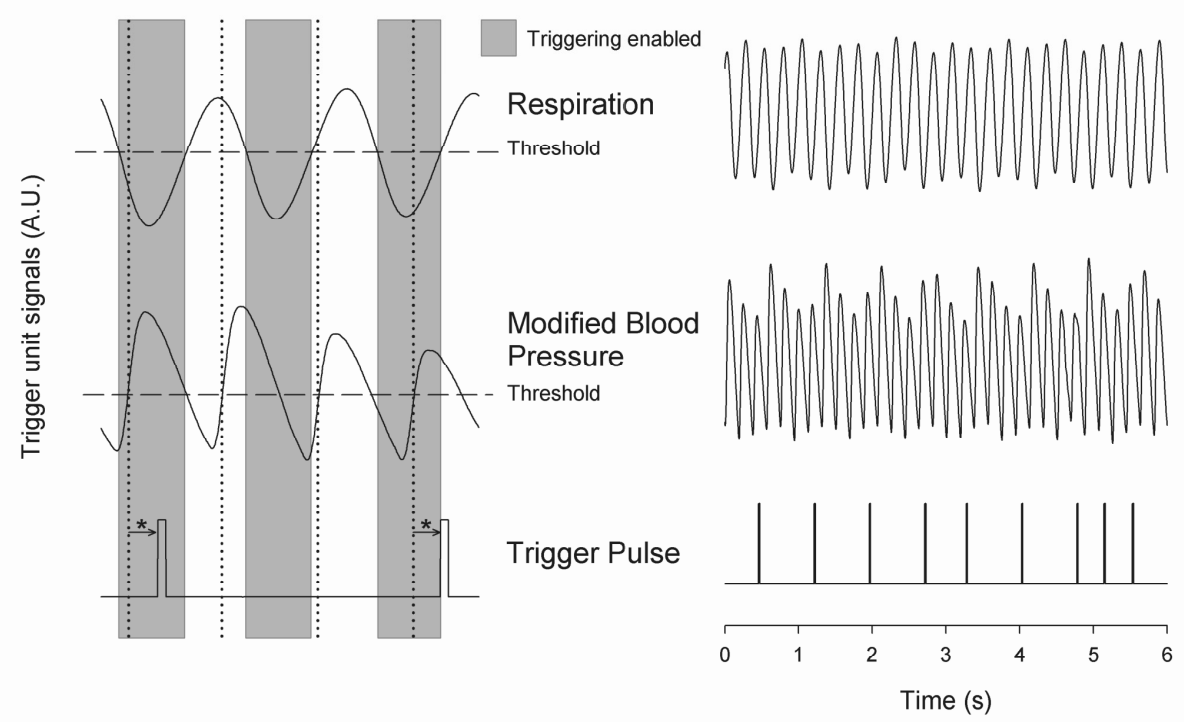

Figure 6.1 Recording of trigger pulse, modified blood pressure and respiration signals.

A recording of the output signals from the trigger unit (modified blood pressure and respiration) and the trigger pulses derived from them. Data were acquired in a ventilated mouse. The blood pressure signal is modified as it is connected to an ECG-input. Nevertheless, it can be used as input for triggering.

Left panel Trigger settings and generation of trigger pulses; thresholds for respiration and modified blood pressure are indicated by horizontal dashed lines. When the respiration signal is below threshold (expiration), triggering is enabled (gray bars). During the first cardiac cycle a trigger pulse is generated, as triggering was enabled when modified blood pressure crossed threshold upwards (vertical dotted line). The next two cardiac cycles, however, did not lead to the generation of a trigger pulse, as the modified blood pressure rose above threshold when triggering was disabled. In the fourth cycle, a trigger pulse is again generated. A trigger delay could be set and is marked by asterisks. The settings for thresholds and delay were determined empirically. Motion artifacts were smallest when imaging in the diastolic phase of the cardiac cycle.

Right panel A recording for the duration of $6 \mathrm{~s}$, clearly showing that trigger pulses are generated in an irregular pattern.

gap between two subsequent optical sections (frames) were recorded using the TPLSM acquisition software (Lasersharp 6.0; Biorad).

\section{Image processing}

Image reconstructions were performed using the Image-Pro Plus 6.0 software (Media Cybernetics Inc., Silver Spring, MD, USA). To further improve overall image quality, interlacing errors were corrected if needed (see results). Furthermore, Fourier transformation was performed to discriminate the original image frequencies (that lie around the origin, i.e., in the corners of the periodic spectrum plot) from higher 
noise and interlacing frequencies. The undesirable higher frequencies were then filtered out by multiplying the Fourier-spectra with a mask based on a twodimensional Gaussian weighted function, thus improving SNR. Filtering in Fourier domain and correction for interlacing inaccuracy were performed using software tools developed with the Mathematica 6.0 software package with "Digital Image Processing" (Wolfram Research Inc., Champaign, III., USA), and the "Front-end Vision" and "MathvisionTools" plugins (Eindhoven University of Technology, the Netherlands).

\section{Results}

\section{Cardiac and respiratory activity}

During in vivo imaging, each image should be acquired well within one cardiac cycle to avoid image distortion (in-frame) and between-frame motion artifacts. In anesthetized mice, the duration of one cardiac cycle varied from 0.16 to $0.24 \mathrm{~s} \mathrm{(4.2} \mathrm{to}$ $6.1 \mathrm{~Hz}$; reference values for nonanesthetized mice: $0.09 \mathrm{~s} / 11.1 \mathrm{~Hz}$ ). This observed cardiac depression is a known side-effect of the applied ketamin/xylazin anesthesia. $^{29}$ In rats anesthetized with urethane (which has less profound effects on heart rate), the cardiac cycle duration varied from 0.25 to $0.30 \mathrm{~s}(3.3$ to $4.0 \mathrm{~Hz}$; reference values $0.17 \mathrm{~s} / 5.9 \mathrm{~Hz}$ ). ${ }^{30}$ Anesthetized animals were ventilated; rats at a rate of 120 $\min ^{-1}(2.0 \mathrm{~Hz})$ and mice at a rate of 200 to $240 \mathrm{~min}^{-1}(3.3$ to $4.0 \mathrm{~Hz})$ with a tidal volume of 200 to $250 \mu \mathrm{l}$. Some mice were allowed to breathe spontaneously, showing an anesthesia-induced respiratory depression over time (mean respiration rate 161 $\pm 35 \mathrm{~min}^{-1}$ at the start of imaging and $134 \pm 32 \mathrm{~min}^{-1}$ at the end of the experiment). Therefore, experimentation time was limited in these animals. On the other hand, arterial movement due to breathing had a lower frequency in these mice and could thus be overcome more easily. Results obtained in artificially damaged carotid arteries were obtained this way. A drawback of unventilated triggering is a less regular respiratory activity, which might impede accurate triggering.

\section{Nontriggered in vivo imaging}

In view of the heart rates already given, image acquisition times should be shorter than $0.16 \mathrm{~s}$ in mice and $0.25 \mathrm{~s}$ in rats in order to limit motion distortion. Such short image acquisition times can be achieved either by increasing the line scan rate or decreasing the number of lines per image (matrix size). The first method, however, decreases the pixel dwell-time at the cost of a lower SNR and, at higher rates, is accompanied by an automatic optical zoom resulting in a smaller FOV (Table 6.1). The second method results in lower pixel resolution, which can only be resolved by 
using an equivalent optical zoom. Comparison of several typical acquisition rates for imaging of an ex vivo mounted artery revealed that imaging at 2 to $3 \mathrm{~Hz}$ yielded optical sections with a sufficient large FOV (no optical zoom is induced) and relatively good overall image quality. However, this frequency was not high enough for in vivo imaging since acquisition time exceeded a complete cardiac cycle and therefore all motions within this cycle were captured in each single image (Figure 6.2 and Video 6.1 (online supplement); mouse carotid artery).

As published before, increasing the image acquisition rate in vivo eliminates large motion artifacts from at least some of the optical sections in a time-series. ${ }^{11}$ Nevertheless, most of the images obtained this way are of low quality due to movements of the sample, loss of focus on the sample, or even complete disappearance of the vessel from the FOV.

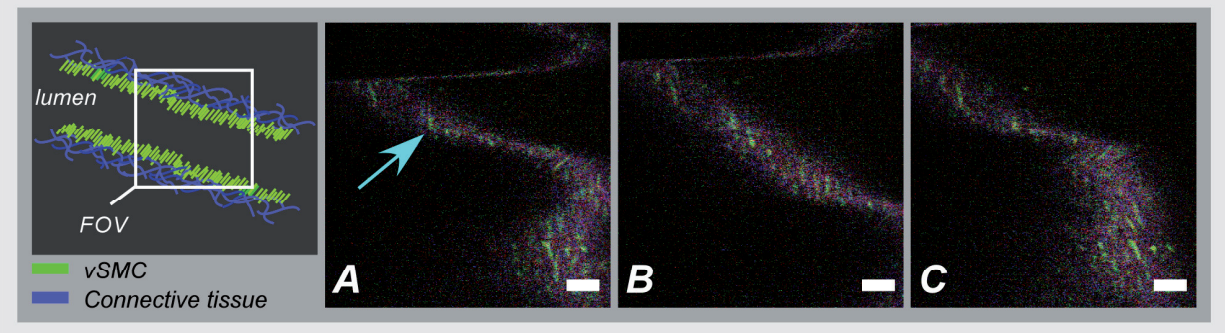

Figure 6.2 Nontriggered in vivo TPLSM at normal (ex vivo) scan rates.

Three subsequent optical sections $(a-c)$ of a left carotid artery of a C57B/6/J mouse obtained in vivo without application of external triggering. Image acquisition time was $0.43 \mathrm{~s} \mathrm{(1200} \mathrm{lines/s;} \mathrm{normal} \mathrm{scan-}$ ning mode; $512 \times 512$ pixels). The schematic drawing on the left indicates the position of the optical sections in the vessel wall; vSMC: vascular smooth muscle cell, FOV: field of view; bars indicate $20 \mu \mathrm{m}$. Cell nuclei (SYTO13, green) and extracellular matrix (SHG of adventitial collagen, blue; autofluorescence of elastin, red) are visible. All sections are disturbed by motion artifacts which cause the arterial wall (blue arrow) to appear as a curved structure. Moreover, every optical section contains different parts of the (moving) vessel wall. The typical morphology and orientation of the smooth muscle cell nuclei (green) is hardly recognizable; the position of the lumen is unclear.

\section{Triggered in vivo imaging}

Triggered image acquisition of each optical section resulted in subsequent images that (almost) all display the same part of the artery during the same phase of movement. Consequently, the obtained optical sections are mutually comparable. As in untriggered imaging, higher frame rates yielded better results than rates in the order of cardiac rates, as images are acquired in a shorter part of the cardiac cycle. Therefore, a more stable period can be found using shorter image acquisition 


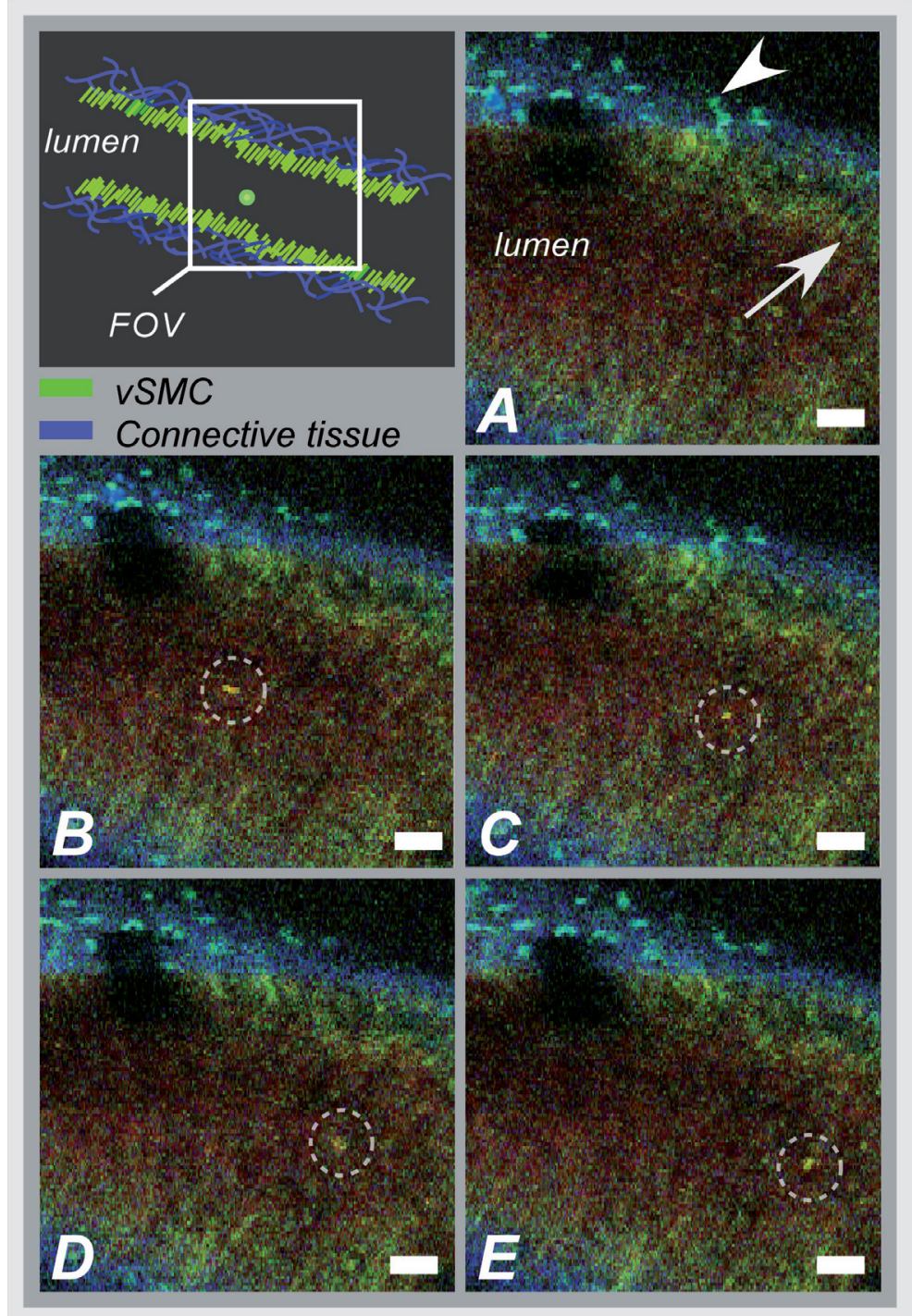

Figure 6.3 Triggered in vivo TPLSM at high scan rates.

Five successive optical sections $(a-e)$ of the left renal artery of a Wistar-Kyoto rat in vivo obtained by triggered acquisition at an image acquisition time of $0.17 \mathrm{~s}$ (1500 lines/s; normal scanning mode; $256 \times 256$ pixels). Cardiac cycle duration was $0.25 \mathrm{~s}$. The schematic drawing displays the position of the optical sections; vSMC: vascular smooth muscle cell, FOV: field of view; bars indicate $20 \mu \mathrm{m}$. Artery was labeled topically for cell nuclei (SYTO13, green) and systemically for cytoplasm of (blood) cells and platelets (acridin red, orange). The successive optical sections contain the same part of the artery. In the tunica adventitia, collagen (SHG, blue) and nuclei (arrowhead) are visible; vSMC nuclei (white arrow) are observable in the tunica media. Moreover, an adhesive blood cell (encircled by white dotted line) is visible against the vessel wall in several $(>10)$ successive images. The lumen appears orange due to circulating unbound acridin red. 


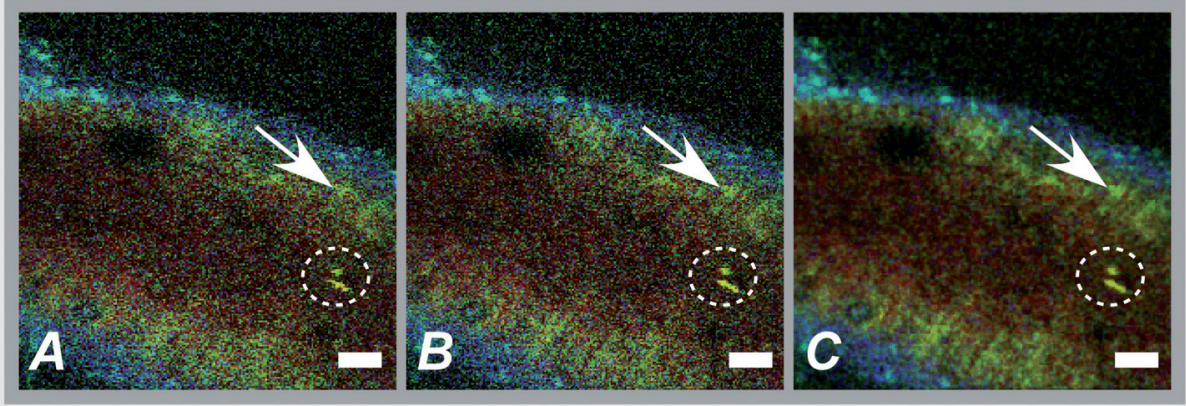

Figure 6.4 Image enhancement.

The effects of image processing applied to one of the optical sections of the series of images as shown in Figure 6.3.

a Raw image of the data file as shown in Figure 6.3; image appears fuzzy and the smooth muscle cell nuclei (white arrow) are hardly recognizable as separate structures.

$b$ Same optical section after correction for inaccurate line positioning (interlacing error). The image already appears sharper and the smooth muscle cell nuclei (white arrow) appear separated. An adhesive blood cell (encircled by white dotted line) is more clearly visible.

$c$ Filtering in the Fourier domain further improves SNR of the image. Also, the nuclei of smooth muscle cells (white arrow) are more apparent.

times (Figure 6.3 and Video 6.2 (online supplement); rat renal artery, image acquisition time $0.17 \mathrm{~s}$; cardiac cycle duration $0.25 \mathrm{~s}$ ).

Series of subsequent optical sections with smallest impact of image distortion and between-frame motion disturbances were obtained when images were acquired within the diastolic phase of the cardiac cycle. However, trigger pulses were generated at peaks of the modified blood pressure signal. Therefore, a trigger delay was used to start image acquisition in diastole, which could easily be introduced via the trigger unit. The amount of delay needed for stable imaging was determined empirically. Timing of image acquisition in (part of) the systolic phase of the cardiac cycle resulted in images with large motion artifacts and strong deformations of the tissue, even at higher imaging rates (not shown). In rat renal arteries, triggered imaging often resulted in more stable images and smaller motion disturbances than in mouse carotid arteries. This finding was to be expected as the more distally located renal artery suffers less from thoracic movement or deformation due to respiration. Also, the heart rate in rats was lower.

\section{Image processing}

Overall image quality suffered from fast acquisition rates that affect the SNR, due to short pixel dwell-times (Figure 6.4a). The SNR was enhanced using Fourierspectrum-based noise filtering (Figure 6.4c). Additionally, fast image acquisition 
using scan rates of $>750$ lines/s (Table 6.1) resulted in images that appeared to exhibit stretched details in horizontal direction. To reduce total acquisition time, odd scan lines of the matrix are automatically scanned from left to right and even scan lines from right to left (bidirectional scanning), instead of scanning each horizontal line from left to right (unidirectional scanning; standard for lower frame rates). Image analysis of bidirectionally scanned images revealed that in comparison with the odd scan lines, the even scan lines of the image matrix were shifted in horizontal direction (varying from 3 to 10 pixels), causing blurred images. Correction of this interlacing inaccuracy resulted in remarkably sharper images (Figure $6.4 \mathrm{~b})$. As a consequence, the image matrix size was also reduced in horizontal direction, reflecting a slightly smaller FOV.

\section{In vivo molecular imaging of exposed subendothelial collagen}

In a subset of experiments, the carotid artery of mice was damaged to expose the thin subendothelial collagen sheet, which was labeled using CNA35-conjugated quantum dots. ${ }^{27}$ Propidium iodide (PI) was used as a cell viability assay. The damaging procedure dramatically increased the number of $\mathrm{PI}$ positive (i.e., nonviable) cells across the vessel wall, including the endothelium (Figures 6.5a and b). Furthermore, subendothelial collagen, which is normally covered by endothelial cells and can not be reached by CNA35-QD525, was now exposed and could be labeled with CNA35-QD525 resulting in a bright green labeled layer. This thin layer was visualized at a subcellular level in vivo, and is clearly located between the smooth muscle and endothelial cell nuclei (Figure 6.5c), which can be distinguished based on morphology and location in the vessel wall. ${ }^{1}$ The collagen in the adventitial layer (as shown by SHG) was not labeled with CNA35-QD525, which indicates that the elastic laminae were still intact and prevented CNA35-QD525 labeling over the vessel wall.

\section{Discussion}

We showed the feasibility of in vivo imaging of structures in the wall of large elastic arteries of mice and rats at a subcellular resolution. Moreover, we visualized the thin collagen sheet present under the endothelium after damaging the vessel wall. It is the first time the subendothelial collagen layer has been imaged in vivo in vessels of this size. Application of fluorescently labeled CNA35 combined with in vivo triggered TPLSM imaging might prove a valuable tool to investigate the role of subendothelial collagen in the onset or progression of (unstable) atherosclerotic plaques. $^{12,28}$ Furthermore, it demonstrates the enormous potential for stable 

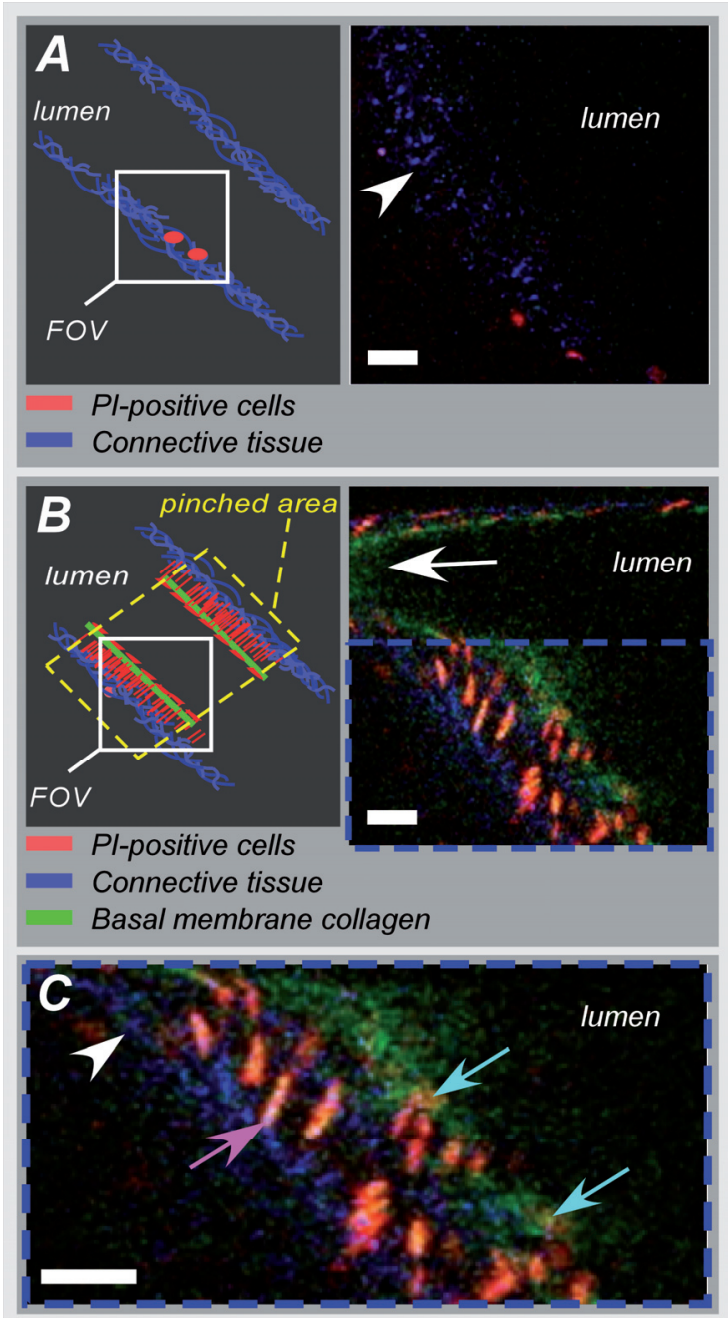

Figure 6.5 Imaging of subendothelial collagen prior to and after mechanical damage. Optical sections of a left carotid artery of a C57Bl6/J mouse obtained in vivo with application of external triggering. Images were recorded at double scanning mode, resulting in decreased spatial resolution in vertical direction ( $y$ direction). Image acquisition time was $0.11 \mathrm{~s}$ (1200 lines/s; double scanning mode; $256 \times 256$ pixels). Cardiac cycle duration was $0.45 \mathrm{~s}$. The schematic drawings on the left indicate the position of the optical sections in the vessel wall; PI propidium iodide, FOV field of view, ROI: region of interest; bars indicate $25 \mu \mathrm{m}$. Mouse carotid artery in vivo after injection of $\mathrm{PI}$ (red) and CNA35-QD525 (green) prior to and after pinching.

a Prepinching; some PI-positive cells (red) can be found in the adventitia (blue SHG, arrowhead) due to surgery.

$b$ Postpinching; the image distortion (white arrow) in the top part indicates that part of the image was not recorded during the diastolic phase; by selecting an ROI (blue dotted area) a stable window can be defined.

$c$ ROI from $b$. Almost all cell nuclei are PI-positive (red; i.e., have a compromised outer cell membrane) and, therefore, a labeled collagen sheet (green) can be observed located between endothelial cell nuclei (round and closest to the lumen; blue arrows) and smooth muscle cell nuclei (elongated; purple arrow). Adventitial collagen is not labeled by quantum dots, but can be identified by SHG (blue, arrowhead).

detection of small vascular structures deep in the tissue, such as subcellular atherosclerotic plaque components, ${ }^{28}$ and the endothelial glycocalyx. ${ }^{31}$

In vivo imaging in larger arteries is severely challenged due to in-frame and between-frame motion artifacts. We showed that a combination of short image acquisition times and triggering on cardiac and respiratory cycles can for the most part overcome these problems (cf., Videos 6.1 and 6.2 in the online supplement). Although image distortion may still occur (Figure 6.5), the influence of betweenframe vessel wall movement on image stability is greatly reduced. 
Triggering on blood pressure signal proved to be most effective to improve image quality as vessel motion is mainly governed by pulse wave deformation (cardiac activity). This is especially true for rat renal arteries. Therefore, application of a more sophisticated solid state pressure transducer catheter (Millar transducer without fluid column, as applied in reference number 32 ), which yields more stable and accurate blood pressure signals, may enhance the precision of triggering and, thus, provide more accurate timing of image acquisition. This would reduce between-frame motion artifacts even further. Image distortion due to vessel motion was reduced by applying shorter image acquisition times (typically $0.17 \mathrm{~s}$ in rat renal arteries and $0.11 \mathrm{~s}$ in mouse carotid arteries). Assuming that the duration of the diastolic phase is about half the cardiac cycle, stable images can be acquired in animals with heart rates varying from 2.9 to $4.5 \mathrm{~Hz}(0.34$ and $0.22 \mathrm{~s}$ per cardiac cycle, resp.) with these settings. If needed, image acquisition times could be shortened to match higher heart rates (Table 6.1). However, there is a trade-off between reduction of distortion and SNR.

Short image acquisition times also dictate small image matrix sizes. As a consequence, pixel resolution is not optimal. Furthermore, high excitation powers are required to generate sufficient signal. This will lead to increased photodegradation of the fluorescent markers, which causes the SNR to drop over time. In the data presented here, however, photodegradation was not observed to hamper experiments significantly. Interestingly, quantum dots are hardly vulnerable to photobleaching, ${ }^{33}$ making them ideal candidates for more lengthy in vivo measurements. The experiments were performed using a combination of different fluorescent markers, each with their own excitation maximum. At the chosen excitation wavelengths ( 800 to $840 \mathrm{~nm}$ ), this meant that the probes could all be excited sufficiently but not optimally. Again, quantum dots are the exception to this rule, as they have broad excitation spectra, with only very small emission peaks.

Theoretically, the axial resolution of our system would be around $0.9 \mu \mathrm{m}$, depending on the excitation wavelength and the NA of the objective used. As discussed elsewhere, ${ }^{1,28}$ a more realistic value would be 1.5 to $2.0 \mu \mathrm{m}$, depending on the location in the vessel. This can for the larger part be explained by a mismatch between the refractive index of the vessel wall components and the objective, which accumulates with increasing depth. Furthermore, the axial resolution will deteriorate when imaging deeper in (scattering) tissue, albeit to a lesser extent than with conventional microscopy. 
Using double or quadruple scanning modes to reduce image acquisition time limited spatial resolution in vertical direction (y direction). Still, even nonoptimally aligned structures could be imaged at a sufficient resolution (Figure 6.5). Applying short image acquisition times also decreased SNR. Using simple and straightforward image processing algorithms, the SNR could be improved. Other image processing techniques could be explored to guarantee optimal post-acquisition SNR improvement. Acquisition SNR can also be improved by narrowing the pulse-width of the excitation light, which would enhance the probability of the two-photon excitation. ${ }^{34}$ As a consequence, signal strength and, thus, SNR increases, which is beneficial for the image quality of accelerated image acquisition. On the other hand, a smaller pulse width would result in increased photodegradation of the fluorescent marker, thus counteracting the increase in signal strength.

Novel high-speed two-photon microscopes ${ }^{22,35}$ enable image acquisition at video rates and will therefore be less hampered by image distortion while providing good SNRs. Still, triggered acquisition is needed to limit between-frame motion artifacts. The present triggering method can easily be applied to these systems.

As already discussed, setting up in vivo experiments based on two-photon microscopy means balancing acquisition speed with heart rate and SNR, excitation wavelength with excitation spectra of fluorescent probes and penetration depth, excitation power with penetration depth and photodegradation (length of experiment). The limitation of the presented system is sampling rate, since the generation of a trigger pulse is dependent both on the cardiac and the respiratory cycle. This means that the delay between images may vary between one and three to four cardiac cycles (Figure 6.1). All taken together, the actual sampling rate of triggered image acquisition in the present system is about 1 to $4 \mathrm{~Hz}$. This is relatively low for imaging of fast functional processes in the vessel wall, such as calcium or nitric oxide dynamics or tracking of moving objects such as blood cells. Nevertheless, slow or stable interactions can still be studied as shown in Figure 6.3. This is also underlined in Video 6.3 (online supplement), acquired from a carotid artery of a ventilated mouse. Cell nuclei were labeled topically and systemically with SYTO13. The video clearly shows cells with lobed nuclei slowly moving in the lumen near the vessel wall. These optical sections were obtained at the end of the experiment, where the blood pressure of the mouse had dropped below $50 \mathrm{mmHg}$, while heart rate was unaltered. Although the physiological relevance of these images should be interpreted with care, they do underline the vast potential of triggered in vivo TPLSM imaging. 


\section{2 | Chapter 6}

In conclusion, the presented method creates new opportunities for in vivo (molecular) imaging at a subcellular level of structures in the (diseased or damaged) arterial wall. Furthermore, three-dimensional imaging of the arterial wall might be possible, especially when the stability and accuracy of triggering on blood pressure is optimized. In addition, it can be used as a platform for in vivo testing and validation of novel molecular agents (e.g., targeted quantum dots ${ }^{33}$ ) as it provides detailed information regarding the behavior of such agents in large arteries in vivo. We showed this tool to be very suitable for CNA35-mediated in vivo collagen imaging.

\section{Acknowledgements}

We thank Evelien Hermeling and Jeroen Hameleers from Maastricht University, Department of Biomedical Engineering, for their help with signal analysis and technical assistance. 


\section{References}

1. Megens RT, Reitsma S, Schiffers PH, Hilgers RH, De Mey JG, Slaaf DW, oude Egbrink MG, van Zandvoort MA. Two-photon microscopy of vital murine elastic and muscular arteries. Combined structural and functional imaging with subcellular resolution. J Vasc Res. 2007;44:87-98.

2. van Gestel MA, Reitsma S, Slaaf DW, Heijnen VV, Feijge MA, Lindhout T, van Zandvoort MA, Elg M, Reneman RS, Heemskerk JW, oude Egbrink MG. Both ADP and Thrombin Regulate Arteriolar Thrombus Stabilization and Embolization, but Are Not Involved in Initial Hemostasis as Induced by Micropuncture. Microcirculation. 2007;14:193-205.

3. oude Egbrink MG, Janssen GH, Ookawa K, Slaaf DW, Reneman RS, Wehrens XH, Maaijwee KJ, Ohshima N, Struijker Boudier HA, Tangelder GJ. Especially polymorphonuclear leukocytes, but also monomorphonuclear leukocytes, roll spontaneously in venules of intact rat skin: involvement of Eselectin. J Invest Dermatol. 2002;118:323-326.

4. Bosman J, Tangelder GJ, oude Egbrink MG, Reneman RS, Slaaf DW. Local application of adenosine induces an increase of capillary diameter in skeletal muscle of anesthetized rabbits. J Vasc Res. 1996;33:111-118.

5. Massberg S, Brand K, Gruner S, Page S, Muller E, Muller I, Bergmeier W, Richter T, Lorenz M, Konrad I, Nieswandt B, Gawaz M. A critical role of platelet adhesion in the initiation of atherosclerotic lesion formation. J Exp Med. 2002;196:887-896.

6. Eriksson EE, Werr J, Guo Y, Thoren P, Lindbom L. Direct observations in vivo on the role of endothelial selectins and alpha(4) integrin in cytokine-induced leukocyte-endothelium interactions in the mouse aorta. Circ Res. 2000;86:526-533.

7. Eriksson EE, Xie X, Werr J, Thoren P, Lindbom L. Direct viewing of atherosclerosis in vivo: plaque invasion by leukocytes is initiated by the endothelial selectins. Faseb J. 2001;15:1149-1157.

8. Huo Y, Schober A, Forlow SB, Smith DF, Hyman MC, Jung S, Littman DR, Weber C, Ley K. Circulating activated platelets exacerbate atherosclerosis in mice deficient in apolipoprotein E. Nat Med. 2003;9:61-67.

9. Murphy DB. Fundamentals of light microscopy and electronic imaging. New York: Wiley-Liss Inc.; 2001.

10. Pawley J. Handbook of biological confocal microscopy. New York: Springer; 2006.

11. van Zandvoort M, Engels W, Douma K, Beckers L, Oude Egbrink M, Daemen M, Slaaf DW. Twophoton microscopy for imaging of the (atherosclerotic) vascular wall: a proof of concept study. $J$ Vasc Res. 2004;41:54-63.

12. Megens RT, Oude Egbrink MG, Cleutjens JP, Kuijpers MJ, Schiffers PH, Merkx M, Slaaf DW, van Zandvoort MA. Imaging collagen in intact viable healthy and atherosclerotic arteries using fluorescently labeled CNA35 and two-photon laser scanning microscopy. Mol Imaging. 2007;6:247-260.

13. Boulesteix T, Pena AM, Pages N, Godeau G, Sauviat MP, Beaurepaire E, Schanne-Klein MC. Micrometer scale Ex Vivo multiphoton imaging of unstained arterial wall structure. Cytometry $A$. 2006;69:20-26.

14. Ferrara DE, Weiss D, Carnell PH, Vito RP, Vega D, Gao X, Nie S, Taylor WR. Quantitative 3D fluorescence technique for the analysis of en face preparations of arterial walls using quantum dot nanocrystals and two-photon excitation laser scanning microscopy. Am J Physiol Regul Integr Comp Physiol. 2006;290:R114-123.

15. Maffia P, Zinselmeyer BH, Ialenti A, Kennedy S, Baker AH, McInnes IB, Brewer JM, Garside P. Images in cardiovascular medicine. Multiphoton microscopy for 3-dimensional imaging of lymphocyte recruitment into apolipoprotein-E-deficient mouse carotid artery. Circulation. 2007;115:e326-328. 
16. Svoboda K, Denk W, Kleinfeld D, Tank DW. In vivo dendritic calcium dynamics in neocortical pyramidal neurons. Nature. 1997;385:161-165.

17. Ashworth SL, Tanner GA. Fluorescent labeling of renal cells in vivo. Nephron Physiol. 2006;103:p9196.

18. Dunn KW, Sandoval RM, Kelly KJ, Dagher PC, Tanner GA, Atkinson SJ, Bacallao RL, Molitoris BA. Functional studies of the kidney of living animals using multicolor two-photon microscopy. Am J Physiol Cell Physiol. 2002;283:C905-916.

19. Dunn KW, Young PA. Principles of multiphoton microscopy. Nephron Exp Nephrol. 2006;103:e3340.

20. Kang JJ, Toma I, Sipos A, McCulloch F, Peti-Peterdi J. Quantitative imaging of basic functions in renal (patho)physiology. Am J Physiol Renal Physiol. 2006;291:F495-502.

21. Sipos A, Toma I, Kang JJ, Rosivall L, Peti-Peterdi J. Advances in renal (patho)physiology using multiphoton microscopy. Kidney Int. 2007;72:1188-1191.

22. Padera TP, Stoll BR, So PT, Jain RK. Conventional and high-speed intravital multiphoton laser scanning microscopy of microvasculature, lymphatics, and leukocyte-endothelial interactions. Mol Imaging. 2002;1:9-15.

23. Palero JA, de Bruijn HS, van der Ploeg van den Heuvel A, Sterenborg HJ, Gerritsen HC. Spectrally resolved multiphoton imaging of in vivo and excised mouse skin tissues. Biophys J. 2007;93:9921007.

24. Palero JA, Latouche G, de Bruijn HS, van der Ploeg van den Heuvel A, Sterenborg HJ, Gerritsen HC. Design and implementation of a sensitive high-resolution nonlinear spectral imaging microscope. $J$ Biomed Opt. 2008;13:044019.

25. Worbs T, Mempel TR, Bolter J, von Andrian UH, Forster R. CCR7 ligands stimulate the intranodal motility of T lymphocytes in vivo. J Exp Med. 2007;204:489-495.

26. Yu W, Braz JC, Dutton AM, Prusakov P, Rekhter M. In vivo imaging of atherosclerotic plaques in apolipoprotein E deficient mice using nonlinear microscopy. J Biomed Opt. 2007;12:054008.

27. Boerboom RA, Krahn KN, Megens RT, van Zandvoort MA, Merkx M, Bouten CV. High resolution imaging of collagen organisation and synthesis using a versatile collagen specific probe. J Struct Biol. 2007;159:392-399.

28. Megens RT, oude Egbrink MG, Merkx M, Slaaf DW, van Zandvoort MA. Two-photon microscopy on vital carotid arteries: imaging the relationship between collagen and inflammatory cells in atherosclerotic plaques. J Biomed Opt. 2008;13:044022.

29. Janssen BJ, De Celle T, Debets JJ, Brouns AE, Callahan MF, Smith TL. Effects of anesthetics on systemic hemodynamics in mice. Am J Physiol Heart Circ Physiol. 2004;287:H1618-1624.

30. Oosting J, Struijker-Boudier HA, Janssen BJ. Autonomic control of ultradian and circadian rhythms of blood pressure, heart rate, and baroreflex sensitivity in spontaneously hypertensive rats. $J \mathrm{Hy}$ pertens. 1997;15:401-410.

31. Reitsma S, Slaaf DW, Vink H, van Zandvoort MA, Oude Egbrink MG. The endothelial glycocalyx: composition, functions, and visualization. Pflugers Arch. 2007;454:345-359.

32. Rensen SS, Niessen PM, van Deursen JM, Janssen BJ, Heijman E, Hermeling E, Meens M, Lie N, Gijbels MJ, Strijkers GJ, Doevendans PA, Hofker MH, De Mey JG, van Eys GJ. Smoothelin-B deficiency results in reduced arterial contractility, hypertension, and cardiac hypertrophy in mice. Circulation. 2008;118:828-836.

33. Prinzen L, Miserus RJ, Dirksen A, Hackeng TM, Deckers N, Bitsch NJ, Megens RT, Douma K, Heemskerk JW, Kooi ME, Frederik PM, Slaaf DW, van Zandvoort MA, Reutelingsperger CP. Optical and magnetic resonance imaging of cell death and platelet activation using annexin a5functionalized quantum dots. Nano Lett. 2007;7:93-100. 
34. McConnell G. Improving the penetration depth in multiphoton excitation laser scanning microscopy. J Biomed Opt. 2006;11:054020.

35. Kurtz R, Fricke M, Kalb J, Tinnefeld P, Sauer M. Application of multiline two-photon microscopy to functional in vivo imaging. J Neurosci Methods. 2006;151:276-286. 

General discussion

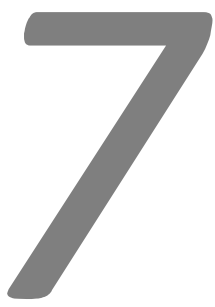


128 Chapter 7 
The aim of this thesis was to explore whether (modification of) the endothelial glycocalyx (EG) plays a role in platelet adhesion in atherogenesis. To this end, an EG imaging and quantification protocol was developed for the mouse carotid artery using two-photon laser scanning microscopy (TPLSM) on mounted carotid arteries. Using this protocol, the EG was assessed as a layer of several micrometers in thickness (Chapter 3). ${ }^{1}$ In the carotid bifurcation of atherogenic mice, the agedependent growth of the EG as seen in control bifurcations was lacking, while at the same time, in vivo platelet adhesion at this site increased with age. This was not seen in the common carotid artery (Chapter 4$){ }^{2}$ Subsequently, other ways to directly visualize the relation between the EG and platelet adhesions were developed. On the one hand, isolated platelets were flushed through mounted carotid arteries, showing that in the carotid bifurcation, but not in the common carotid artery, breakdown of the EG results in increased levels of platelet adhesion (Chapter 5). On the other, the feasibility of in vivo TPLSM imaging of small structures (about $1 \mu \mathrm{m}$ ) within the vessel wall of large arteries was demonstrated. This approach could ultimately be applied to EG visualization in the future, provided that a suitable in vivo EG labeling method becomes available (Chapter 6). ${ }^{3}$

The general hypothesis underlying our work is that atherogenic stimuli disturb the balance between EG production and shedding at predilection sites for atherosclerosis, resulting in a reduction of the local EG thickness. This would in turn increase the likelihood of platelet adhesion, thus contributing to atherogenesis. The data presented in this thesis show that in reality the matter is more complicated. Especially from the data in Chapters 4 and 5 , several factors governing platelet adhesion can be deduced. They are summarized below and in Figure 7.1.

\section{Influence of flow profile}

In control animals, hardly any platelet adhesion was observed in the common carotid artery in vivo, which is also found in our flow model in vessels with low entrance lengths (Figures 4.2 and 5.5). Exposure of the isolated common carotid artery to nonaxial flow increases platelet adhesion, possibly due to the fact that platelets were propelled into the EG. Such radial platelet movement into the EG is compliant with an estimated low stiffness of the EG. ${ }^{4}$ It is unlikely that EG thickness in these areas was acutely reduced. As such, the flow profile could be identified as factor for platelet adhesion. 


\section{Influence of the endothelial glycocalyx}

The carotid bifurcation is exposed to nonaxial flow. Although most flow deviation occurs downstream in the side branches, flow in the area just proximal of the flow divider region will deviate to a certain extent as well. ${ }^{5}$ This induces radial movement of platelets, making the bifurcation prone to platelet adhesion. However, in bifurcations of control mice, platelet adhesion levels were low and comparable to that of the common carotid artery, both in vivo and in mounted arteries with low entrance lengths. At the same time, the bifurcation was covered by a relatively thick EG which is apparently able to prevent platelet adhesion in the presence of nonaxial flow (Figure 4.2). In our flow model (Chapter 5), reduction of EG thickness by an enzymatic challenge increased platelet adhesion significantly in the bifurcation. Because atherogenic stimuli are known to reduce EG thickness in vivo as well (Chapter 2), this may explain the increased levels of platelet adhesion in the carotid bifurcation of $\mathrm{ApoE}^{-/-}$mice. In contrast, enzymatic reduction of $\mathrm{EG}$ thickness in the common carotid artery did not induce platelet adhesion in case of axial flow (Figure 5.5). Therefore, the EG is probably a factor in platelet adhesion, especially in areas exposed to nonaxial flow.

\section{Influence of platelet pre-activation}

In our ex vivo model, platelet adhesion in areas subjected to axial flow was correlated to the entrance length (Figure 5.4). Pre-activation of platelets due to a long entrance length resulted in higher levels of adhesion and even aggregation downstream. This effect was not site-specific. In atherogenic conditions platelets become activated due to (prolonged) exposure to hyperlipidemia. ${ }^{6}$ It is likely that platelet activation in atherogenic mice contributed to the higher levels of platelet adhesion in vivo. However, the magnitude of this effect can be debated, as in vivo platelet adhesion in the common carotid artery of atherogenic mice did not differ from that in controls (Figure 4.2).

\section{Influence of age - role of endothelium}

Platelet adhesion in atherogenic mice increased significantly with age (Figure 4.3). This age effect can (partly) be explained by prolonged exposure of platelets to increasing hyperlipidemia (Table 4.1), leading to pre-activation of platelets, and, hence, adhesion. On the other hand, the endothelium was exposed to increasing hyperlipidemia as well, leading to activation of endothelial cells. ${ }^{7}$ As a consequence, the balance between production and shedding of EG components is disturbed, ${ }^{9-12}$ which decreases EG density and increases EG permeability. This in turn results in an increased likelihood of platelet adhesion to the vessel wall. As such, 


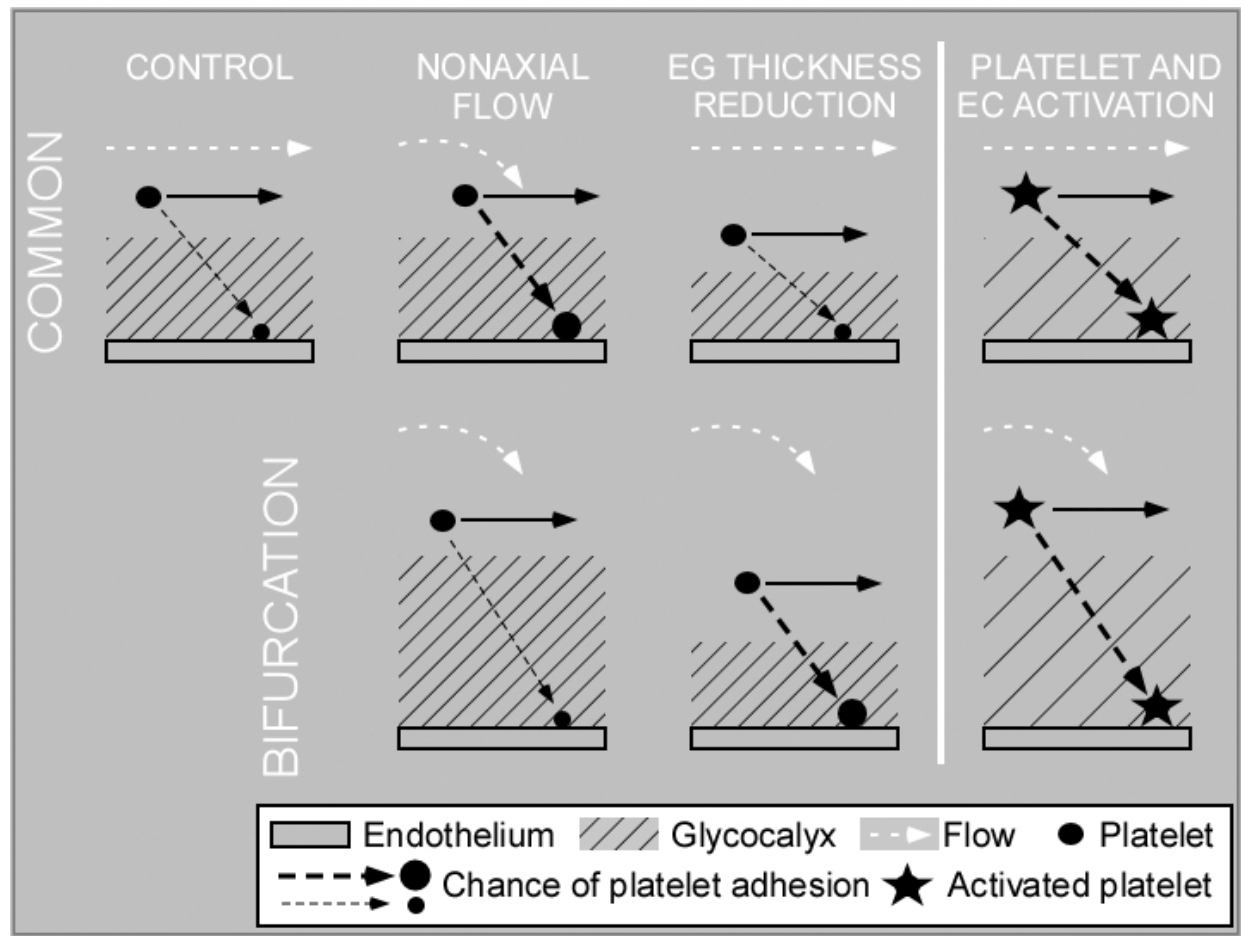

Figure 7.1 Factors in platelet adhesion.

Simplified schematic representation of our hypothesis concerning various conditions that determine platelet adhesion. The figure is not drawn to scale. The chance of platelet adhesion is indicated by the dashed arrows directed towards the endothelium; the thicker this line and the larger the dot near the endothelium, the higher the change of platelet adhesion. Note that the endothelial glycocalyx in the bifurcation is thicker than in the common carotid artery. The density of the glycocalyx is represented by the density of oblique lines. The various situations are deduced from experimental data. For more information, see the given Figures from the experimental chapters.

Common from left to right: Control Figures 4.2 and 5.5; Nonaxial flow Figures 5.3 and 5.5; EG thickness reduction Figure 5.5; Platelet and EC activation Figures 5.4 and 5.5.

Bifurcation from left to right: Control (axial flow) is not available as the bifurcation is always subjected to nonaxial flow (to a certain extent). Nonaxial flow Figures 4.2 and 5.5; EG thickness reduction Figures 4.2 and 5.5; Platelet and EC activation Figures 5.4 and 5.5. It is likely that EG density also decreased with decreasing EG thickness, but this could not be determined in our experiments.

EG endothelial glycocalyx; EC endothelial cell; common common carotid artery; bifurcation carotid artery bifurcation.

endothelial cell activation (with age) could explain both the absence of growth of the EG and the increased levels of platelet adhesion seen in atherogenic mice (Figure 4.3).

It remains to be elucidated to what extent platelet and/or endothelial cell activation is causally involved in atherogenesis, and to what extent interplay exists be- 
tween these effects, as activated platelets have the capacity to activate endothelial cells and vice versa. ${ }^{8}$

\section{Future perspectives}

With the techniques developed and described in this thesis it is possible to investigate the effects of therapeutical interventions on (platelet adhesion in) atherogenesis. We hypothesize that strategies increasing EG thickness and/or density at atherogenesis-prone sites will lead to decreased levels of platelet adhesion, a less severe inflammatory response and a lower plaque burden. Selection of suitable strategies can be performed using the flow model as described in Chapter 5, while long-term effects can be explored in vivo as described in Chapters 4 and 6.

One of the major disadvantages of the current labeling protocol of the EG is the limited penetration of wheat germ agglutinin into the glycocalyx in control conditions, making it unsuitable for use in the in vivo situation (Figure 3.2). As shown in Chapter 6, the microscopy setup already allows stable in vivo imaging in larger arteries of mice. The identification of a lectin or other label suited as a general label for the EG in vivo, would enable visualization of the glycocalyx at sites of platelet and/or leukocyte adhesion. This would allow direct observation of the role of the EG in these processes, and serve as a platform to assess the effect of (therapeutical) interventions. However, the time-resolution of in vivo TPLSM is relatively low (Figure 6.1). The use of state-of-the-art commercially available high speed twophoton microscopes is a promising development in this perspective. ${ }^{20,21}$

Assessment of EG thickness alone is not sufficient as it does not unequivocally reflect its functional quality. Therefore, other parameters should be explored in more detail in our model, such as glycocalyx density, glycocalyx composition, ${ }^{13-16}$ as well as other functional measures, such as (deviating) flow patterns, vessel wall thrombogenicity, ${ }^{17}$ intimal thickening and accumulation of specific molecules, ${ }^{13,18}$ and nitric oxide production in response to shear. ${ }^{19}$

\section{Conclusions}

In this thesis we show that the endothelial glycocalyx of the carotid artery is a layer of several micrometers thick. In areas subjected to nonaxial flow, platelet adhesion increases when the EG is challenged by (prolonged) exposure to hyperlipidemia or hyaluronidase. In this way, the EG appears to be a modulator of platelet adhesion at atherogenesis-prone sites. Although platelet adhesion is governed by more factors than the EG alone, restoration of glycocalyx structure in an atherogenic environment might well ameliorate the development of atherosclerotic plaques. 


\section{References}

1. Reitsma S, oude Egbrink MGA, Vink H, van den Berg B, Lima Passos V, Engels W, Slaaf DW, van Zandvoort MAMJ. Endothelial glycocalyx structure in the intact carotid artery: a two-photon laser scanning microscopy study. J Vasc Res. 2011;48:297-306.

2. Reitsma S, Oude Egbrink MG, Heijnen VV, Megens RT, Engels W, Vink H, Slaaf DW, van Zandvoort MA. Endothelial glycocalyx thickness and platelet-vessel wall interactions during atherogenesis. Thromb Haemost. 2011;106:939-946.

3. Megens RT, Reitsma S, Prinzen L, oude Egbrink MG, Engels W, Leenders PJ, Brunenberg EJ, Reesink $\mathrm{KD}$, Janssen BJ, ter Haar Romeny BM, Slaaf DW, van Zandvoort MA. In vivo high-resolution structural imaging of large arteries in small rodents using two-photon laser scanning microscopy. J Biomed Opt. 2010;15:011108.

4. Han YF, Weinbaum S, Spaan JAE, Vink H. Large-deformation analysis of the elastic recoil of fibre layers in a Brinkman medium with application to the endothelial glycocalyx. Journal of Fluid Mechanics. 2006;554:217-235.

5. Ku DN, Giddens DP. Pulsatile flow in a model carotid bifurcation. Arteriosclerosis. 1983;3:31-39.

6. Cadroy Y, Lemozy S, Diquelou A, Ferrieres J, Douste-Blazy P, Boneu B, Sakariassen KS. Human type II hyperlipoproteinemia enhances platelet-collagen adhesion in flowing nonanticoagulated blood. Arterioscler Thromb. 1993;13:1650-1653.

7. Sima AV, Stancu CS, Simionescu M. Vascular endothelium in atherosclerosis. Cell Tissue Res. 2009;335:191-203.

8. Dole VS, Bergmeier W, Mitchell HA, Eichenberger SC, Wagner DD. Activated platelets induce Weibel-Palade-body secretion and leukocyte rolling in vivo: role of P-selectin. Blood. 2005;106:2334-2339.

9. Constantinescu A, Spaan JA, Arkenbout EK, Vink H, Vanteeffelen JW. Degradation of the endothelial glycocalyx is associated with chylomicron leakage in mouse cremaster muscle microcirculation. Thromb Haemost. 2011;105:790-801.

10. Constantinescu AA, Vink H, Spaan JA. Endothelial cell glycocalyx modulates immobilization of leukocytes at the endothelial surface. Arterioscler Thromb Vasc Biol. 2003;23:1541-1547.

11. Nieuwdorp M, Mooij HL, Kroon J, Atasever B, Spaan JA, Ince C, Holleman F, Diamant M, Heine RJ, Hoekstra JB, Kastelein JJ, Stroes ES, Vink H. Endothelial glycocalyx damage coincides with microalbuminuria in type 1 diabetes. Diabetes. 2006;55:1127-1132.

12. Nieuwdorp M, Holleman F, de Groot E, Vink H, Gort J, Kontush A, Chapman MJ, Hutten BA, Brouwer CB, Hoekstra JB, Kastelein JJ, Stroes ES. Perturbation of hyaluronan metabolism predisposes patients with type 1 diabetes mellitus to atherosclerosis. Diabetologia. 2007;50:1288-1293.

13. van den Berg BM, Spaan JA, Vink H. Impaired glycocalyx barrier properties contribute to enhanced intimal low-density lipoprotein accumulation at the carotid artery bifurcation in mice. Pflugers Arch. 2009;457:1199-1206.

14. Meyer W, Godynicki S, Tsukise A. Lectin histochemistry of the endothelium of blood vessels in the mammalian integument, with remarks on the endothelial glycocalyx and blood vessel system nomenclature. Ann Anat. 2008;190:264-276.

15. Accili D, Menghi G, Gabrielli MG. Lectin histochemistry for in situ profiling of rat colon sialoglycoconjugates. Histol Histopathol. 2008;23:863-875.

16. Henry $\mathrm{CB}$, DeFouw DO. Differential lectin binding to microvascular endothelial glycoconjugates during normal angiogenesis in the chick chorioallantoic membrane. Microvasc Res. 1995;49:201211. 
17. Nagy N, Freudenberger T, Melchior-Becker A, Rock K, Ter Braak M, Jastrow H, Kinzig M, Lucke S, Suvorava T, Kojda G, Weber AA, Sorgel F, Levkau B, Ergun S, Fischer JW. Inhibition of hyaluronan synthesis accelerates murine atherosclerosis: novel insights into the role of hyaluronan synthesis. Circulation. 2010;122:2313-2322.

18. van den Berg BM, Spaan JA, Rolf TM, Vink H. Atherogenic region and diet diminish glycocalyx dimension and increase intima-to-media ratios at murine carotid artery bifurcation. Am J Physiol Heart Circ Physiol. 2006;290:H915-920.

19. Mochizuki S, Vink H, Hiramatsu O, Kajita T, Shigeto F, Spaan JA, Kajiya F. Role of hyaluronic acid glycosaminoglycans in shear-induced endothelium-derived nitric oxide release. Am J Physiol Heart Circ Physiol. 2003;285:H722-726.

20. Fan GY, Fujisaki H, Miyawaki A, Tsay RK, Tsien RY, Ellisman MH. Video-rate scanning two-photon excitation fluorescence microscopy and ratio imaging with cameleons. Biophys J. 1999;76:24122420.

21. Drechsler M, Megens RT, van Zandvoort M, Weber C, Soehnlein O. Hyperlipidemia-triggered neutrophilia promotes early atherosclerosis. Circulation. 2010;122:1837-1845. 
Summary

Samenvatting

About the author

Dankwoord 
136 Chapter 8 


\section{Summary}

The circulation of blood is of pivotal importance to homeostasis of the body. Circulating plasma and blood cells support cell and organ metabolism. The smallest blood cells, blood platelets (about 1-2 $\mu \mathrm{m}$ ), are mainly known for their role in aggregation and coagulation in the case of vessel damage. However, platelets are involved in many other processes as well. One of the pathological conditions to which platelets contribute is atherosclerosis.

Atherosclerosis is a wide-spread condition in Western societies, which is marked by the formation of so-called plaques in the vessel wall of larger arteries at predilection sites (e.g., arterial bifurcations) under conditions of hyperlipidemia. It is regarded to be an inflammatory process. The formation of these plaques (atherogenesis) starts in the first decades of life. Early during atherogenesis platelets adhere to the vessel wall of larger arteries and contribute to the inflammatory response leading to plaque formation. It is unknown what property of the endothelium attracts platelets to these predilection sites. In this thesis, the possibility is explored that changes in the endothelial glycocalyx, the luminal cover of endothelial cells, underlie platelet adhesion in early atherogenesis.

Chapter 2 provides an overview of the current knowledge on the structure and function of the endothelial glycocalyx, which appears to be actively involved in many processes affecting the vessel wall, such as vascular permeability, blood cellvessel wall interactions, thrombogenicity and mechanotransduction. In this chapter, several techniques for visualization and quantification of the endothelial glycocalyx are addressed as well.

Chapter 3 describes our newly developed ex vivo imaging and quantification protocol for assessing the thickness and coverage of the endothelial glycocalyx in the isolated mouse carotid artery (diameter about $500 \mu \mathrm{m}$ ), flushed with a fluorescently labeled lectin. Imaging was performed through the vessel wall using twophoton laser scanning microscopy. This methodological protocol leads to less damage to the glycocalyx during the preparation process than most other reported preparation and visualization techniques. The thickness of the common carotid artery endothelial glycocalyx as measured in this way appeared to be about $2.3 \mu \mathrm{m}$. The lectin-based approach provides a general impression of the glycocalyx, as approximately $90 \%$ of the endothelial surface area was labeled in the common carotid 
artery. Interestingly, in the atherogenesis-prone internal carotid artery only about $70 \%$ of the endothelial surface area was labeled, while the thickness of the labeled glycocalyx was comparable $(2.5 \mu \mathrm{m})$. This suggests that spatial differences in the glycocalyx can be related to a different propensity of the vessel wall for (platelet adhesion in) atherogenesis.

In Chapter 4, the effect of hyperlipidemia on both platelet-vessel wall interactions and endothelial glycocalyx thickness in the atherogenesis-prone carotid artery bifurcation is explored using intravital microscopy and ex vivo two-photon microscopy approaches in atherogenic mice and wild-type controls. In control animals, hardly any adhesion of platelets to the wall of the carotid artery bifurcation was seen, regardless of age. The thickness of the endothelial glycocalyx at this location was $2.5 \mu \mathrm{m}$ on average. Interestingly, in controls glycocalyx thickness increased with age from $2.4 \mu \mathrm{m}$ in young mice to $3.0 \mu \mathrm{m}$ in aged ones. In atherogenic mice higher levels of platelet adhesion were observed, with a clear age-dependency (from 24 platelets/mm2 in young to 64 platelets/mm2 in aged mice). In contrast, the age-dependent growth of the endothelial glycocalyx as seen in controls was lacking. As a result, the thickness of the glycocalyx in the atherogenic bifurcation was constant at about $2.2 \mu \mathrm{m}$ and, as such, less thick than in control bifurcations. This lack of growth of glycocalyx might explain the increasing numbers of platelet adhesion to the vessel wall.

However, glycocalyx dimensions in the atherogenic bifurcation were still comparable to those found in the common carotid artery of both atherogenic mice $(1.9 \mu \mathrm{m})$ and wild-type controls $(2.2 \mu \mathrm{m})$, while hardly any platelet adhesions occurred in the common carotid artery in either strain. In other words, no straight-forward relation between the thickness of the endothelial glycocalyx and the number of plateletvessel wall interactions was observed.

To gain more insight in the possible role of the glycocalyx in platelet adhesion in the carotid artery, an ex vivo flow model was developed, which is described in Chapter 5. Platelet solutions were flushed through isolated, mounted carotid arteries, while wide-field fluorescence microscopy was applied to visualize plateletvessel wall interactions in the common carotid artery and carotid artery bifurcation. Interestingly, the flow profile needed a certain length of the proximal common carotid artery to develop into a pattern with axially oriented flow lines. The area exposed to developing flow (entrance length) was prone to platelet-vessel wall interactions. Longer entrance lengths induced platelet pre-activation, which resulted in high numbers of platelet adhesion ( $>160$ platelets $/ \mathrm{mm} 2$ ) as well as 
aggregation downstream. In vessels with low platelet pre-activation hardly any platelet adhesions were observed either in the common carotid artery or the carotid bifurcation. This is comparable to data from the in vivo studies presented in Chapter 4. Enzymatic breakdown of the endothelial glycocalyx in these vessels increased platelet adhesion in the bifurcation (nonaxial flow) to 32 platelets $/ \mathrm{mm} 2$, while in the common carotid artery (axial flow) no effect of reduction of the endothelial glycocalyx thickness could be observed. This way, a relation between the local flow profile (axial or nonaxial), the thickness of the endothelial glycocalyx and the number of platelet adhesions could be established. Thus, the endothelial glycocalyx acts as a modulator of platelet adhesion in areas exposed to nonaxial flow. Therefore, the previously described reduction in glycocalyx thickness at the carotid bifurcation of atherogenic mice (Chapter 4) might explain the increase in platelet adhesion at this site during atherogenesis.

To further address the role of the endothelial glycocalyx in atherogenesis, an in vivo imaging method is required. Chapter 6 reports that it is possible to image thin layers in the vessel wall using two-photon laser scanning microscopy in vivo. The key to stable imaging in these vessels was found to be the combination of adjusted imaging speeds and triggered image acquisition based on the respiratory and cardiac cycles. As a proof of principle, the subendothelial collagen layer (about $1 \mu \mathrm{m}$ ) was fluorescently labeled and stably visualized at an acceptable sampling rate (about $1-4 \mathrm{~Hz}$ ). Although the fluorescent labeling protocol for the glycocalyx used in this thesis is not applicable in vivo, the techniques described in this chapter form the basis for in vivo imaging of the endothelial glycocalyx in large arteries in the future.

The results of this thesis are discussed in Chapter 7, thus giving an overview of new insights provided by our work on the role of the endothelial glycocalyx in platelet adhesion during early atherogenesis. 


\section{Samenvatting}

Veel mensen in onze westerse cultuur ontwikkelen gedurende hun leven de ziekte vaatverkalking, ook wel bekend als atherosclerose. Dit is een aandoening waarbij er in grote bloedvaten (slagaders) een ontstekingsproces op gang komt. Hierbij hoopt ontstekingsmateriaal zich op in de wand van het bloedvat en vormt een zogenaamde plaque. Het kan na verloop van tijd voorkomen dat een dergelijke plaque scheurt, waardoor er een acute stollingsreactie van het bloed optreedt, met vorming van bloedproppen. Dit leidt dan tot aandoeningen als een hart- of herseninfarct. Daarmee vormt atherosclerose een van de grootste onderliggende oorzaken van sterfte in de westerse beschaving.

Om de ontwikkeling van atherosclerotische plaques goed te kunnen begrijpen, is inzicht in de structuur en functie van de wand van slagaders essentieel. Deze vaatwand kent drie lagen. De buitenste laag bestaat uit bindweefsel en bindweefselvormende cellen, en zorgt hiermee voor de verankering van het vat in zijn omgeving. De middelste laag bestaat uit spiercellen omgeven door elastisch bindweefsel. Hierdoor kan het vat enerzijds oprekken en anderzijds een bepaalde weerstand bieden als er bloed met een hoge druk doorheen wordt gepompt. De binnenste laag bestaat uit zogenaamde endotheelcellen. Deze zorgen er onder andere voor dat bloed in het vat niet gaat stollen.

In dit proefschrift wordt een specifiek onderdeel van de vaatwand belicht: de endotheliale glycocalyx. Dit is een laag met speciale suiker- en eiwitdeeltjes die vanuit de endotheelcellen groeit en in contact staat met stromend bloed. Doordat er vocht en andere deeltjes vanuit het bloed de glycocalyx binnendringen, ontstaat er een soort gelei. Een overzicht over de huidige kennis van de structuur en functie van de endotheliale glycocalyx wordt gegeven in hoofdstuk 2. In hoofdstuk 3 wordt aangetoond dat de endotheliale glycocalyx in intacte halsslagaders van muizen door middel van geavanceerde twee-foton microscopie in beeld gebracht kan worden. In deze vaten blijkt de endotheliale glycocalyx enkele micrometers dik te zijn. Ter vergelijking: de onderliggende endotheelcellen zijn zelf meestal niet eens een halve micrometer dik!

We hebben in dit proefschrift ook nagedacht over een functioneel belang van de endotheliale glycocalyx. Hierbij hebben we gekeken naar het gedrag van bloedplaatjes. Bloedplaatjes zijn de kleinste cellen in het bloed (ca. 1 micrometer in 
doorsnee) en zijn betrokken bij het vormen van een stolsel in geval van schade aan een bloedvat. In normale omstandigheden blijven bloedplaatjes in het stromende bloed aanwezig zonder in contact te komen met de vaatwand. In de wetenschappelijke literatuur was reeds beschreven dat bloedplaatjes vroeg in het ontstaan van atherosclerotische plaques aan de vaatwand van slagaders blijven plakken (adhesie). Wanneer hun binding aan de vaatwand wordt voorkomen, worden de plaques minder groot. De vraag komt dan op waarom bloedplaatjes blijven plakken? En ook: hoe is dit mogelijk als er een glycocalyx 'in de weg zit' die enkele malen groter is dan het bloedplaatje zelf? Om hier meer inzicht in te krijgen hebben we zowel het gedrag van bloedplaatjes als de dikte van de glycocalyx bestudeerd bij gezonde muizen en bij muizen die plaques ontwikkelen. De resultaten van deze studies worden in hoofdstuk 4 beschreven. Op plaatsen waar zich plaques vormen is de glycocalyx van de zieke dieren dunner terwijl er daar meer bloedplaatjes blijven plakken. Deze bevinding pleit voor een beschermende rol van de glycocalyx. Toch blijkt de zaak iets ingewikkelder. Op plaatsen waar geen plaques ontstaan werden geen plakkende bloedplaatjes gevonden, terwijl hier de glycocalyx even dun was als op plekken waar wel plaques ontstaan in zieke dieren. Er lijkt derhalve geen eenduidige relatie te bestaan tussen de dikte van de glycocalyx en de hoeveelheid plakkende bloedplaatjes.

Om meer duidelijkheid te krijgen over de rol van de glycocalyx in bloedplaatjesadhesie hebben we een model ontwikkeld waarbij zowel de halsslagaders als bloedplaatjes uit muizen werden geïsoleerd (hoofdstuk 5). De slagaders werden in een vaatbakje opgespannen, waarna de bloedplaatjes erdoorheen werden gespoeld. Tegelijkertijd werd met een microscoop naar het plakgedrag van de bloedplaatjes gekeken. Hierbij bleek dat er op plekken waar zich plaques vormen een verstoord stroomprofiel is, wat inhoudt dat de stromingsrichting niet volledig parallel aan de vaatwand loopt. Afbraak van de glycocalyx op deze plekken leidde wel tot een verhoging van het aantal plakkende bloedplaatjes, terwijl dit op plaatsen met een normaal (parallel) stroomprofiel niet het geval was. Uit deze experimenten konden we concluderen dat de glycocalyx wel degelijk een beschermende rol lijkt te hebben door op plaatsen met een verstoord stroomprofiel bloedplaatjesadhesie tegen te gaan.

Dit inzicht kan de basis zijn waarop we in de toekomst mogelijk met behulp van glycocalyx-versterkende strategieën (zoals medicijnen) de ontwikkeling van vaatverkalking tegen kunnen gaan. Om het succes van dergelijke interventies goed te kunnen evalueren is er een manier nodig om de structuur van de glycocalyx in het levende dier te onderzoeken. In hoofdstuk 6 wordt een opzet hiertoe beschreven. We laten zien dat het mogelijk is om zeer fijne structuren (laagjes van een micro- 
meter dik) in grote slagaders van muizen en ratten in beeld te brengen. De grootste uitdaging hierbij is om deze structuren onbewogen af te beelden, ondanks de natuurlijke beweging van het bloedvat ten gevolge van de hartslag en de ademhaling. Deze beweging zorgt ervoor dat de structuur waarin we geïnteresseerd zijn voortdurend beweegt en steeds uit beeld verdwijnt. Dit effect kan sterk verminderd worden door het moment van afbeelding op een gunstig moment tijdens de hartslag en ademhaling te laten plaatsvinden. Ook beschrijven we welke winst verwacht mag worden van het veranderen van instellingen van de twee-foton microscopie opstelling.

Tot slot wordt in hoofdstuk 7 een algemene uiteenzetting gegeven van de belangrijkste bevindingen in dit proefschrift en van de wetenschappelijke waarde hiervan. Dit alles mondt uit in een beschrijving van de verschillende factoren die van invloed zijn op plaatjesadhesie aan de wand van grote slagaders en de rol van de endotheliale glycocalyx hierin. 


\section{About the author}

Sietze Reitsma was born on October $24^{\text {th }} 1982$ in Amsterdam, the Netherlands. He finished secondary education (VWO/Gymnasium) with honours in 2000 at Scholengemeenschap St. Ursula, Horn. A son of two general practitioners, he studied Medicine at Maastricht University from 2000 to 2009. During this period, he participated in scientific work in the lab of Dr. M.G.A. oude Egbrink, department of Physiology. In 2003, he obtained his status of 'Article 9' - expert according to the Law on Animal experiments. Through the years, he combined medical internships with research as a PhD-student under direct supervision of Dr. M.A.M.J. van Zandvoort, department of Biomedical Engineering. As such, he successfully followed several courses on basic scientific skills and techniques provided by the Cardiovascular Research Institute Maastricht (CARIM). He attended several national and international congresses as well. After becoming a medical doctor in September 2009, Sietze began his ENT-specialization at the Maastricht University Medical Centre in December 2009. He is still working as a resident today and striving to become an ENT-specialist in 2015.

In 2005, Sietze married Hanneke Stegeman. They live in Reuver together with their two children Eline (2009) and Matthijs (2011). 


\section{List of publications}

- Platelet-vessel wall interactions in the isolated mouse carotid artery - roles for flow profile entrance length and endothelial glycocalyx.

Reitsma S, van Zandvoort M, Vink H, Slaaf D, oude Egbrink M.

Submitted

- Endothelial glycocalyx thickness and platelet-vessel wall interactions during atherogenesis.

Reitsma S, oude Egbrink M, Heijnen V, Megens R, Engels W, Vink H, Slaaf D, van Zandvoort M. Thromb Haemost. 2011 Nov;106(5):939-46.

- Endothelial glycocalyx structure in the intact carotid artery: a two-photon laser scanning microscopy study.

Reitsma S, oude Egbrink M, Vink H, van den Berg B, Passos V, Engels W, Slaaf D, van Zandvoort M. J Vasc Res. 2011;48(4):297-306.

- In vivo high-resolution structural imaging of large arteries in small rodents using two-photon laser scanning microscopy.

Megens R, Reitsma S, Prinzen L, oude Egbrink M, Engels W, Leenders P, Brunenberg E, Reesink K, Janssen B, ter Haar Romeny B, Slaaf D, van Zandvoort M.

J Biomed Opt. 2010 Jan-Feb;15(1):011108.

- Complementary roles of platelets and coagulation in thrombus formation on plaques acutely ruptured by targeted ultrasound treatment: a novel intravital model.

Kuijpers MJ, Gilio K, Reitsma S, Nergiz-Unal R, Prinzen L, Heeneman S, Lutgens E, van Zandvoort M, Nieswandt B, oude Egbrink M, Heemskerk J.

J Thromb Haemost. 2009 Jan;7(1):152-61.

- Both ADP and thrombin regulate arteriolar thrombus stabilization and embolization, but are not involved in initial hemostasis as induced by micropuncture.

van Gestel M, Reitsma S, Slaaf D, Heijnen V, Feijge M, Lindhout T, van Zandvoort M, Elg M, Reneman R, Heemskerk J, oude Egbrink M.

Microcirculation. 2007 Apr-May;14(3):193-205.

- Two-photon lifetime imaging of fluorescent probes in intact blood vessels: a window to subcellular structural information and binding status.

Douma K, Megens R, Reitsma S, Prinzen L, Slaaf D, Van Zandvoort M.

Microsc Res Tech. 2007 May;70(5):467-75.

- The endothelial glycocalyx: composition, functions, and visualization.

Reitsma S, Slaaf D, Vink H, van Zandvoort M, oude Egbrink M.

Pflugers Arch. 2007 Jun;454(3):345-59.

- Two-photon microscopy of vital murine elastic and muscular arteries. Combined structural and functional imaging with subcellular resolution.

Megens R, Reitsma S, Schiffers P, Hilgers R, De Mey J, Slaaf D, oude Egbrink M, van Zandvoort M. J Vasc Res. 2007;44(2):87-98. 


\section{Dankwoord}

Toen ik in de beginjaren van mijn studie Geneeskunde wat ging 'klussen' bij de vakgroep Fysiologie had ik niet durven vermoeden dat er een dergelijk proefschrift uit voort zou komen. Het begon er allemaal mee dat ik werd aangesproken: "Hey, een nieuw gezicht! Wie ben jij, en... we zoeken nog een student-assistent... interesse?" Voor ik het wist zat ik in een sollicitatiegesprek. Kort daarna begon een periode waarin ik mocht groeien in de wereld die wetenschap heet. Van het één kwam telkens het ander: helpen met data-analyse, helpen met experimenten, proefdierkundecursus, keuzeblokken, een promotietraject vol experimenteren, discussiëren, analyseren, her-analyseren, interpreteren, her-her-analyseren, schrijven, insturen, reviseren...... In dit geheel ben ik door veel mensen steeds verder geholpen. Een aantal van hen wil ik expliciet bedanken.

Prof. Slaaf, Dick, ik wil je danken voor je bijdrage aan mijn promotieproces. We hebben wellicht even aan elkaar moeten wennen, maar gaandeweg heb ik steeds meer respect gekregen voor je intellect, je trouw en je bereidheid me verder te helpen. Wellicht ben ik de hekkensluiter van een lange serie, en ik hoop dat je trots kunt zijn op dit boekwerkje.

Prof. oude Egbrink, Mirjam, je hebt me van het begin af aan weten te enthousiasmeren voor de wetenschap. Je bent hierin een echte 'MoEke' geweest. Ik ben je zeer erkentelijk voor je tijd, je interesse, en je geduld. Je gave om in een oogopslag een zin of een alinea te verbeteren heeft me meer dan eens gered.

Prof. van Zandvoort, Marc, wat ik aan jou heel erg waardeer is je mogelijkheid om zingend een experiment op te zetten, en daarbij buiten de vaste kaders te denken. Je bent altijd zeer geïnteresseerd geweest, niet in de laatste plaats in mijn persoonlijke leven. Vaak zaten we te kletsen en moesten de data aan het eind even snel de revue passeren. Je deur heeft altijd open gestaan, ook in perioden dat je het zelf niet altijd makkelijk had. Ik hoop nog vaak op je bank te kunnen ploffen.

Prof. Vink, Hans, het is jammer dat we het niet voor elkaar hebben kunnen krijgen om je als co-promotor aan te merken. Ook jij bent altijd zeer betrokken geweest en hebt me als 'glycocalyx-goeroe' meer dan eens verbaasd met een nieuw gezichtspunt. Jouw grootste kracht is je goede humeur en je talent om 'saaie' data tot leven te brengen door een originele interpretatie. Meer dan eens ging ik bedroefd over mijn resultaten je kamer in, en kwam ik er geïnspireerd weer uit.

Door de jaren heen heb ik veel tijd mogen besteden met diverse collegae. Het begon op het 'kippenhok' van Fysiologie. Miriam en Anita, dank voor de gezelligheid 
en de bereidwilligheid om een 'groen' studentje een goed voorbeeld te geven van promoveren (en van Sokoban spelen...). Toen ik zelf aan mijn promotietraject begon, werd ik ingelijfd bij het 'Twee-Foton-Team'. Remco, ik wil je danken voor de manier waarop je me als 'Two-photon-Master' hebt ingewijd in het opspannen en imagen van vaatjes. Het heeft ons o.a. een aantal gezamenlijke publicaties opgeleverd. Daarnaast heb je altijd veel lol en een goed gesprek gezocht. Dank dat je mijn paranimf wilde zijn. Lenneke, dank voor je kritische blik en hulp waar nodig. Kim, dank voor je bijdrage in ons team. Wim, zelden heb ik zo'n energieke wervelwind ontmoet. Je hield op veel fronten de boel draaiende. Dank voor je inzet en steun. De overige leden van Biofysica/Biomedische Technologie, onder wie Timo, Mitrajit, Arnold, Theo, Jeroen, Anneke, Liselotte, Evelien, Dennis, Joost, Koen en Wilco, wil ik danken voor de tijd dat ik deel van 'de boel' mocht zijn. Marijke en Imke, ook jullie bedankt voor jullie bijdrage aan het geheel, zowel op het vakinhoudelijke als op het gezellige vlak.

Viviane, zonder jou was dit avontuur nooit begonnen. We hebben heel wat uren doorgebracht op een zeer prettige manier. Je bent direct betrokken geweest bij de meeste resultaten in dit boekje. Heel fijn dat je wilde optreden als mijn paranimf.

Ook mijn huidige KNO-collegae wil ik bedanken. Mijn gebazel over de glycocalyx of over intravitaal microscopie heeft menig wenkbrauw doen bewegen tijdens de journal clubs. Mijn immer irritante statistiek opmerkingen nemen jullie daarnaast op de koop toe. In het bijzonder dank aan mijn opleiders, prof. Kremer en prof. Stokroos. Bernd en Robert, dank dat jullie me de tijd hebben gegeven om dit promotietraject tot een goed einde te brengen.

Mijn (schoon)familie wil ik ook noemen. Pa en ma, zonder jullie aanhoudende steun en jullie hulpvaardigheid was ik nooit zover gekomen. Dank voor jullie liefde in raad en daad. Mijn schoonouders: dank voor jullie oprechte interesse in een ingewikkeld onderwerp. Dank dat jullie aan een kwibus met zulke verhalen je dochter hebben toevertrouwd. Broer, (schoon)zussen en zwagers: dank voor jullie interesse ondanks alles (muizen enzo...)

Hanneke, als iemand een ereplaats verdient in dit dankwoord, dan ben jij het wel! Je hebt vanaf het begin van dichtbij meegemaakt hoe dit proefschrift en de inhoud daarvan tot stand is gekomen. Je hebt alle verhalen aangehoord, me regelmatig moeten missen tijdens een van mijn meetsessies of congressen, mijn worstelingen en frustraties gezien en me daarin telkens gesteund. Ook heb je samen met me 
kunnen genieten van de (kleine) successen: een publicatie eindelijk geaccepteerd, of een experiment eindelijk doorgrond. Het is zeker in de laatste jaren niet altijd makkelijk geweest als ik naast het KNO-bestaan ook nog met mijn hoofd in 'glycocalyx-land' was. Je hebt veel voor me opgevangen waardoor ik ruimte had om tot dit resultaat te komen. Daarvoor ben ik je intens dankbaar. Het is daarmee net zo goed jouw prestatie! Ik hou ontzettend veel van je.

Mijn Hemelse Vader wil ik ook danken voor alle manieren waarop Hij mij geleid heeft in dit proces. Heer, $U$ bent de Schepper van hemel en aarde. Wat is het heerlijk dat $U$ mij de kans gaf om intensief bezig te zijn met een minuscuul deeltje van Uw oneindige mooie schepping. Dank U!

Het moeilijkst aan het schrijven van een dankwoord is wellicht het overwinnen van de angst om iemand te vergeten. Daarom zou ik willen afsluiten met een gevierde uitspraak van een burgervader van een niet nader te noemen (kinder-)TVprogramma:

"Iedereen die heeft geholpen aan de totstandkoming van dit proefschrift: bedankt! ledereen die niet heeft geholpen: ook bedankt!" 
"Sit finis libri, non finis quaerendi" 